Supporting Information

for

\title{
Zirconocene-Mediated Highly Regio- and Stereoselective Synthesis of Multisubstituted Olefins Starting from 1-Alkynylboronates
}

\author{
Yasushi Nishihara, * Mitsuru Miyasaka, Masanori Okamoto, Hideki Takahashi, \\ Eiji Inoue, Kenki Tanemura, and Kentaro Takagi
}

Division of Chemistry and Biochemistry, Graduate School of Natural Science and Technology, Okayama University, 3-1-1 Tsushimanaka, Okayama 700-8530, Japan

Phone: +81-86-251-7855

$$
\text { Fax: }+81-86-251-7855
$$

Email: ynishiha@cc.okayama-u.ac.jp

1. General

2. Experimental Procedures and Spectroscopic Data for New Compounds

3. Copies of ${ }^{1} H$ and ${ }^{13} C$ NMR Charts for the New Compounds 
1. General. All the reactions were carried out under an Ar atmosphere using standard Schlenk techniques. Glassware was dried in an oven $\left(130^{\circ} \mathrm{C}\right)$ and heated under reduced pressure before use. Dehydrated toluene, dichloromethane, hexane, and diethyl ether were purchased from Kanto Chemicals Co., Ltd. For thin layer chromatography (TLC) analyses throughout this work, Merck precoated TLC plates (silica gel $60 \mathrm{GF}_{254}, 0.25$ $\mathrm{mm}$ ) were used. Silica gel column chromatography was carried out using Silica gel $60 \mathrm{~N}$ (spherical, neutral, 40-100 m) from Kanto Chemicals Co., Ltd. NMR spectra $\left({ }^{1} \mathrm{H},{ }^{13} \mathrm{C}\left\{{ }^{1} \mathrm{H}\right\},{ }^{11} \mathrm{~B}\left\{{ }^{1} \mathrm{H}\right\}\right.$, and $\left.{ }^{19} \mathrm{~F}\left\{{ }^{1} \mathrm{H}\right\}\right)$ were recorded on Varian INOVA-600 (600 MHz) or Mercury-300 (300 MHz) spectrometers. Peak positions of the ${ }^{11} \mathrm{~B}\left\{{ }^{1} \mathrm{H}\right\}$ NMR spectra were referenced to an external $\mathrm{BF}_{3} \mathrm{OEt}_{2}$. Infrared spectra were recorded on a Shimadzu IRPrestige-21 spectrophotometer. GC analyses were performed on a Shimadzu GC-14A equipped with a flame ionization detector using Shimadzu Capillary Column (CBP1-M25-025) and Shimadzu C-R6A-Chromatopac integrator. Melting Points were measured on a Yanagimoto micromelting point apparatus and are uncorrected. The GC yields were determined using suitable hydrocarbon internal standards. GC/MS analyses were carried out on a SHIMADZU GC-17A equipped with a SHIMADZU QP-5050 GC-MS system. Elemental analyses were carried out with a Perkin-Elmer $2400 \mathrm{CHN}$ elemental analyzer at Osaka City University.

\section{Experimental procedures and Spectroscopic Data for New Compounds}

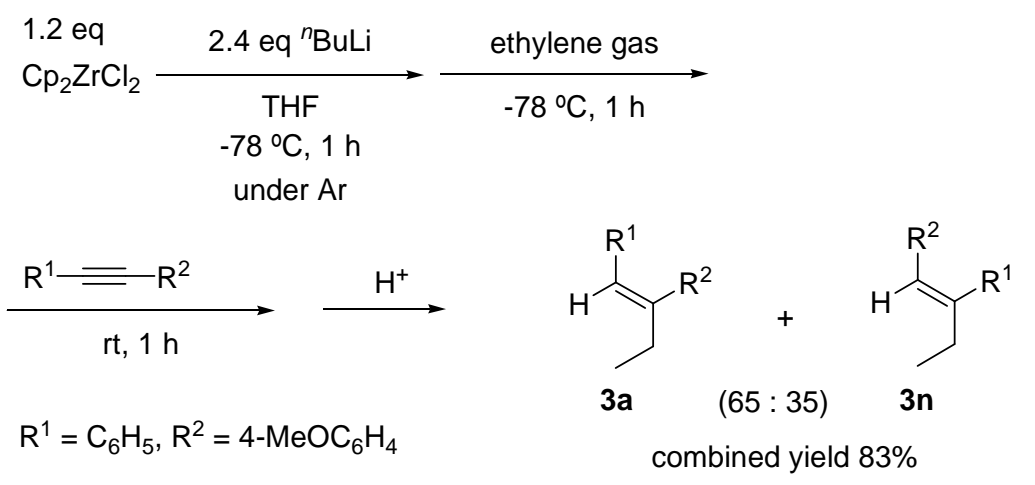

Regioselectivity upon Formation of Zirconacyclopentene using Unsymmetrical Diarylethyne. To a solution of zirconocene dichloride (702 mg, $2.4 \mathrm{mmol})$ in THF (12 mL) was added dropwise butyllithium (3 $\mathrm{mL}, 4.8 \mathrm{mmol}, 1.6 \mathrm{M}$ hexane solution) at $-78{ }^{\circ} \mathrm{C}$. The reaction mixture was stirred for $1 \mathrm{~h}$ at $-78{ }^{\circ} \mathrm{C}$ and then atmospheric ethylene gas was introduced into a vessel at $-78{ }^{\circ} \mathrm{C}$. The reaction mixture was warmed to room temperature and 1-(methoxyphenyl)-2-phenylethyne $(417 \mathrm{mg}, 2.0 \mathrm{mmol})$ was added. The reaction mixture was stirred for $1 \mathrm{~h}$, quenched with $1.0 \mathrm{M} \mathrm{HCl}$, and extracted with diethyl ether. Organic layer was separated and the 
aqueous layer was extracted with diethyl ether. The combined ethereal layers were washed with brine, and dried over $\mathrm{MgSO}_{4}$. Filtration and concentration under vacuum, followed by purification with bulb to bulb distillation $\left(180^{\circ} \mathrm{C} / 2\right.$ Torr) gave a mixture of $3 \mathbf{a}$ and $3 \mathbf{n}$ (combined yield was $83 \%$, 3a: $\mathbf{3 n}=65: 35$ determined by ${ }^{1} \mathrm{H}$ NMR and GC measurements). Regio- and stereochemistry of the synthesized compounds $3 \mathbf{a}$ and $\mathbf{3 n}$ was determined by comparison of spectroscopic data of authentic samples obtained in this paper.

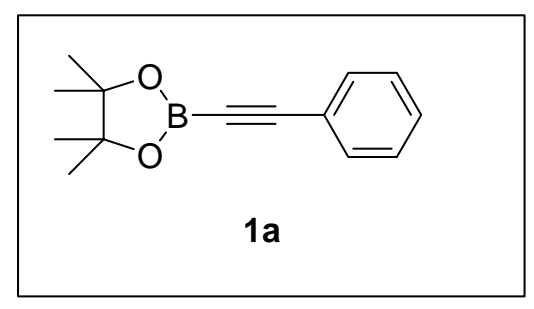

General Procedure for 1-Alkynyldioxaborolanes 1: Synthesis of 4,4,5,5-Tetramethyl-2(phenylethynyl)-1,3,2-dioxaborolane (1a). ${ }^{1} \quad$ To a solution of phenylacetylene $(1.32 \mathrm{~mL}, 12 \mathrm{mmol})$ in THF $(30 \mathrm{~mL})$ in a $50 \mathrm{~mL}$ of Schlenk tube $-78^{\circ} \mathrm{C}$ under an $\mathrm{Ar}$ atmosphere were added dropwise n-BuLi $(7.5 \mathrm{~mL}, 1.6$ $\mathrm{M}$ hexane solution, $12 \mathrm{mmol}$ ). The reaction mixture was stirred for $1 \mathrm{~h}$ at $-78{ }^{\circ} \mathrm{C}$. The resulting reaction mixture was then added to a solution of 4,4,5,5-tetramethyl-2-(1-methylethoxy)-1,3,2-dioxaborolane (2.04 mL, $10 \mathrm{mmol})$ in THF $(30 \mathrm{~mL})-78^{\circ} \mathrm{C}$. After being stirred for $2 \mathrm{~h}$ at $-78^{\circ} \mathrm{C}$, the reaction mixture was quenched with $1.0 \mathrm{M} \mathrm{HCl} / \mathrm{Et}_{2} \mathrm{O}(12.6 \mathrm{~mL}, 12.6 \mathrm{mmol})$, and the mixture was warmed to room temperature with additional $1 \mathrm{~h}$ stirring. Filtration and evaporation afforded a pale yellow oil. Bulb to bulb distillation $\left(160{ }^{\circ} \mathrm{C} / 2\right.$ Torr $)$ gave 1a $\left(2.22 \mathrm{~g}, 9.72 \mathrm{mmol}, 97 \%\right.$ yield) as white solid. Mp. 58-60 ${ }^{\circ} \mathrm{C}$. FT-IR (neat, $\left.\mathrm{cm}^{-1}\right): 2981(\mathrm{~m}), 2195$ $(v(\mathrm{C}=\mathrm{C}), \mathrm{w}), 1461(\mathrm{~m}), 1388(\mathrm{~m}), 1372(\mathrm{~m}), 1130(\mathrm{~m}), 692(\mathrm{~s}) .{ }^{1} \mathrm{H}$ NMR $\left(\mathrm{CDCl}_{3}, 300 \mathrm{MHz}, \mathrm{rt}\right) \delta 1.33(\mathrm{~s}, 12 \mathrm{H})$, 7.26-7.36 (m, 3H), 7.51-7.54 (m, 2H); ${ }^{13} \mathrm{C}\left\{{ }^{1} \mathrm{H}\right\} \mathrm{NMR}\left(\mathrm{CDCl}_{3}, 75 \mathrm{MHz}, \mathrm{rt}\right): \delta 24.6,84.4,101.7,121.8,128.2$, 129.4, 132.5. The carbon signal attached to B was not observed due to low intensity; ${ }^{11} \mathrm{~B} \mathrm{NMR}\left(\mathrm{CDCl}_{3}, 96\right.$ MHz, rt) $\delta$ 24.05. MS (EI, m/z (relative intensity)): 228 (M+34), 213 (23), 170 (33), 155 (18), 143 (74), 129 (100), 124 (13), 102 (12), 85 (16), 77 (28).

(1) Gandon, V.; Leca, D.; Aechtner, T.; Vollhardt, K. P. C.; Malacria, M.; Aubert, C. Org. Lett. 2004, 6, 34053407. 


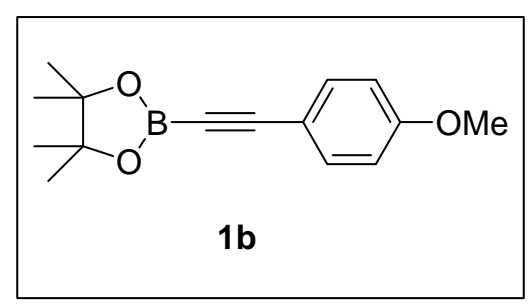

2-[(4-Methoxyphenyl)ethynyl]-4,4,5,5-tetramethyl-1,3,2-dioxaborolane (1b). White solid. Yield: 89\% (2.29 g, $8.88 \mathrm{mmol})$. Bp. $190^{\circ} \mathrm{C} / 2$ Torr. Mp. $72-74{ }^{\circ} \mathrm{C}$. FT-IR (neat, $\left.\mathrm{cm}^{-1}\right): 2976(\mathrm{~m}), 2184(v(\mathrm{C} \equiv \mathrm{C}), \mathrm{w}), 1383$ (m), 1249 (s), 1140 (m), 845 (s). ${ }^{1} \mathrm{H}$ NMR $\left(\mathrm{CDCl}_{3}, 300 \mathrm{MHz}, \mathrm{rt}\right) \delta 1.31$ (s, 12H), 3.80 (s, 3H), 6.82 (d, $J=9.9$, 2H), $7.47(\mathrm{~d}, J=9.9,2 \mathrm{H}) ;{ }^{13} \mathrm{C}\left\{{ }^{1} \mathrm{H}\right\} \mathrm{NMR}\left(\mathrm{CDCl}_{3}, 75 \mathrm{MHz}, \mathrm{rt}\right): \delta 24.6,55.2,84.3,102.1,113.8,113.9,134.2$, 160.4. The carbon signal attached to $\mathrm{B}$ was not observed due to low intensity; ${ }^{11} \mathrm{~B} \mathrm{NMR}\left(\mathrm{CDCl}_{3}, 96 \mathrm{MHz}, \mathrm{rt}\right)$ $\delta$ 23.94. MS (EI, m/z (relative intensity)): $258\left(\mathrm{M}^{+}, 80\right), 243$ (18), 199 (14), 173 (83), 158 (100), 143 (13), 128 (12), 115 (28), 89 (10), 85 (12). HRMS Calcd for $\mathrm{C}_{15} \mathrm{H}_{19} \mathrm{BO}_{3}: 258.1427$. Found: $\mathrm{M}^{+}, 258.1422$.

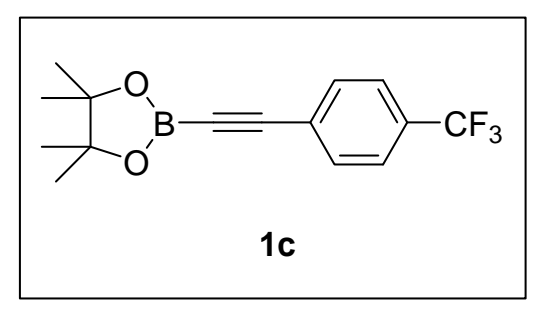

2-[(4-Trifluoromethylphenyl)ethynyl]-4,4,5,5-tetramethyl-1,3,2-dioxaborolane (1c). White solid. Yield: $66 \%$ (1.96 g, $6.63 \mathrm{mmol})$. Bp. $150{ }^{\circ} \mathrm{C} / 2$ Torr. Mp. $113-114{ }^{\circ} \mathrm{C}$. FT-IR (neat, $\left.\mathrm{cm}^{-1}\right): 2988(\mathrm{~m}), 2199(v(\mathrm{C} \equiv \mathrm{C})$, w), $1382(\mathrm{~m}), 1376(\mathrm{~m}), 1138(\mathrm{~m}), 841(\mathrm{~s}) .{ }^{1} \mathrm{H} \mathrm{NMR}\left(\mathrm{CDCl}_{3}, 300 \mathrm{MHz}, \mathrm{rt}\right) \delta 1.33(\mathrm{~s}, 12 \mathrm{H}), 7.58(\mathrm{~d}, J=8.4$, $2 \mathrm{H}), 7.63(\mathrm{~d}, J=8.4,2 \mathrm{H}) ;{ }^{13} \mathrm{C}\left\{{ }^{1} \mathrm{H}\right\} \mathrm{NMR}\left(\mathrm{CDCl}_{3}, 75 \mathrm{MHz}, \mathrm{rt}\right): \delta 24.6,84.7,99.8,123.7(\mathrm{q}, J=270.6 \mathrm{~Hz})$, $125.2(\mathrm{q}, J=3.7 \mathrm{~Hz}), 125.6,131.0(\mathrm{q}, J=32.5 \mathrm{~Hz}), 132.6$. The carbon signal attached to B was not observed due to low intensity; ${ }^{11} \mathrm{~B} \mathrm{NMR}\left(\mathrm{CDCl}_{3}, 96 \mathrm{MHz}, \mathrm{rt}\right) \delta 23.89 ;{ }^{19} \mathrm{~F} \mathrm{NMR}\left(\mathrm{CDCl}_{3}, 282 \mathrm{MHz}, \mathrm{rt}\right)-63.52$. MS (EI, m/z (relative intensity)): $296\left(\mathrm{M}^{+}, 28\right), 281$ (39), 238 (23), 211 (56), 197 (100), 177 (25), 169 (27), 141 (13), 124 (27), 85 (29). Anal. Calcd for $\mathrm{C}_{15} \mathrm{H}_{16} \mathrm{~F}_{3} \mathrm{BO}_{2}$ : C, 60.85; H, 5.45\%. Found: C, 60.65; H, 5.43\%. 


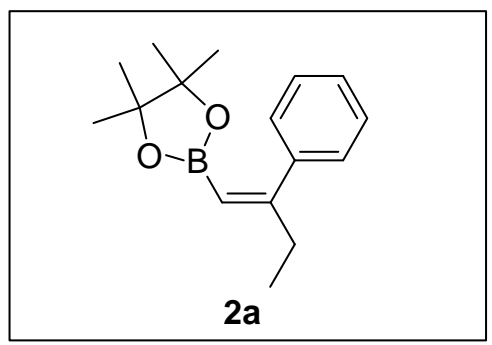

General Procedure for Zirconocene-Mediated Regio- and Stereoselective Ethylation of 1a-1c: Formation of (Z)-4,4,5,5-Tetramethyl-2-(2-phenyl-1-buten-1-yl)-1,3,2-dioxaborolane (2a). To a solution zirconocene dichloride $(7.02 \mathrm{~g}, 24 \mathrm{mmol})$ in THF $(120 \mathrm{~mL})$ in a $300 \mathrm{~mL}$ of two-necked flask under an Ar atmosphere were added dropwise $\mathrm{n}-\mathrm{BuLi}\left(30 \mathrm{~mL}, 48 \mathrm{mmol}, 1.6 \mathrm{M}\right.$ hexane solution) at $-78{ }^{\circ} \mathrm{C}$. After the reaction mixture was stirred for $1 \mathrm{~h}$ at $-78^{\circ} \mathrm{C}$, atmospheric ethylene gas was introduced into the vessel for $1 \mathrm{~h}$ at $-78^{\circ} \mathrm{C}$. The reaction mixture was warmed to room temperature and 4,4,5,5-tetramethyl-2-(phenylethynyl)1,3,2-dioxaborolane (1a) was added. The mixture was stirred for $1 \mathrm{~h}$, quenched with $1 \mathrm{M}$ hydrochloric acid (50 $\mathrm{mL})$, and extracted with diethyl ether $(25 \mathrm{~mL} \times 2)$. The combined ethereal layer was washed with brine and dried over $\mathrm{MgSO}_{4}$. Filtration and evaporation afforded yellow oil. Bulb to bulb distillation $\left(150{ }^{\circ} \mathrm{C} / 2 \mathrm{Torr}\right)$ gave 3a (4.21 g, $16.3 \mathrm{mmol}, 80 \%$ yield) as pale yellow oil. FT-IR (neat, $\left.\mathrm{cm}^{-1}\right): 2933(\mathrm{~m}), 1628(\mathrm{w}), 1461(\mathrm{~m})$, $1379(\mathrm{~m}), 1371$ (m), 1146 (m), 740 (s), 698 (s). ${ }^{1} \mathrm{H}$ NMR $\left(\mathrm{CDCl}_{3}, 300 \mathrm{MHz}, \mathrm{rt}\right): \delta 1.04$ (t, J=7.4 Hz, 3H), $1.12(\mathrm{~s}, 12 \mathrm{H}), 2.49(\mathrm{q}, J=7.4 \mathrm{~Hz}, 2 \mathrm{H}), 5.45(\mathrm{~s}, 1 \mathrm{H}), 7.24-7.28(\mathrm{~m}, 5 \mathrm{H}) ;{ }^{13} \mathrm{C}\left\{{ }^{1} \mathrm{H}\right\} \mathrm{NMR}\left(\mathrm{CDCl}_{3}, 75 \mathrm{MHz}, \mathrm{rt}\right): \delta$ 12.5, 24.5, 24.8, 33.3, 82.9, 127.1, 127.5, 127.8, 143.1, 163.6; ${ }^{11} \mathrm{~B}$ NMR ( $\left.\mathrm{CDCl}_{3}, 96 \mathrm{MHz}, \mathrm{rt}\right) \delta 30.02$. MS (EI, m/z (relative intensity)): $258\left(\mathrm{M}^{+}, 51\right), 201$ (39), 172 (17), 158 (69), 143 (100), 129 (25), 117 (24), 115 (16), 105 (51), 101 (24). Anal. Calcd for $\mathrm{C}_{16} \mathrm{H}_{23} \mathrm{BO}_{2}$ : C, 74.44; H, 8.98\%. Found: C, 74.11; H, 8.98\%.

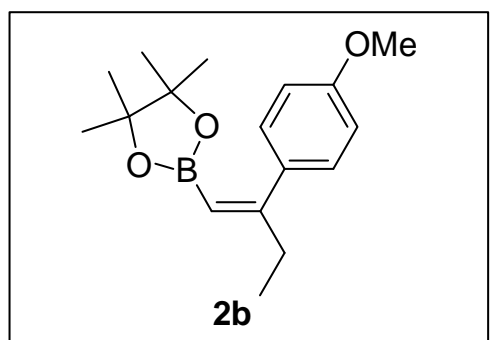

(Z)-4,4,5,5-Tetramethyl-2-[2-(4-methoxyphenyl)-1-buten-1-yl]-1,3,2-dioxaborolane (2b). Yellow solid, Yield: 72\% (4.15 g, $14.4 \mathrm{mmol})$. Bp. $180^{\circ} \mathrm{C} / 2$ Torr. Mp. 42-43 ${ }^{\circ} \mathrm{C}$. FT-IR $\left(\mathrm{CHCl}_{3}, \mathrm{~cm}^{-1}\right): 2979$ (m), 2935 (m), 2837 (m), 1622 (w), 1381 (m), 1372 (m), 1246 (s), 1109 (m), 835 (s). ${ }^{1} \mathrm{H}$ NMR $\left(\mathrm{CDCl}_{3}, 300 \mathrm{MHz}, \mathrm{rt}\right) \delta$ 
$1.03(\mathrm{t}, J=7.2 \mathrm{~Hz}, 3 \mathrm{H}), 1.11(\mathrm{~s}, 12 \mathrm{H}), 2.47(\mathrm{q}, J=7.4 \mathrm{~Hz}, 2 \mathrm{H}), 3.81(\mathrm{~s}, 3 \mathrm{H}), 5.38(\mathrm{~s}, 1 \mathrm{H}), 6.81(\mathrm{~d}, J=8.7 \mathrm{~Hz}$, $2 \mathrm{H}), 7.20(\mathrm{~d}, J=9.0 \mathrm{~Hz}, 2 \mathrm{H}) ;{ }^{13} \mathrm{C} \mathrm{NMR}\left(\mathrm{CDCl}_{3}, 75 \mathrm{MHz}, \mathrm{rt}\right) \delta 12.7,24.6,24.8,33.3,55.2,82.9,112.9,129.0$, $135.4,159.0,163.2 ;{ }^{11} \mathrm{~B} \mathrm{NMR}\left(\mathrm{CDCl}_{3}, 96 \mathrm{MHz}, \mathrm{rt}\right) \delta 30.18 ;{ }^{19} \mathrm{~F} \mathrm{NMR}\left(\mathrm{CDCl}_{3}, 282 \mathrm{MHz}, \mathrm{rt}\right) \delta$-62.9. MS (EI, m/z (relative intensity)): 288 (M+ 55), 231 (11), 188 (18), 172 (100), 160 (10), 147 (15), 135 (22), 129 (7), 101 (11), 83 (6). Anal. Calcd for $\mathrm{C}_{17} \mathrm{H}_{25} \mathrm{BO}_{3}$ : C, 70.85; H, 8.74\%. Found: C, 70.65; H, 8.78\%.

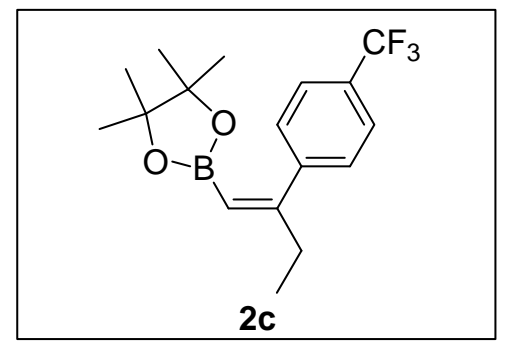

(Z)-4,4,5,5-Tetramethyl-2-[2-(4-trifluoromethyphenyl)-1-buten-1-yl]-1,3,2-dioxaborolane (2c). Pale yellow oil. Yield: $68 \%(4.44 \mathrm{~g}, 13.6 \mathrm{mmol})$. Bp. $140{ }^{\circ} \mathrm{C} / 2$ Torr. FT-IR (neat, $\left.\mathrm{cm}^{-1}\right): 2978(\mathrm{~m}), 2935(\mathrm{~m})$, $1631(\mathrm{w}), 1380(\mathrm{~m}), 1371$ (m), $1126(\mathrm{~m}) .{ }^{1} \mathrm{H} \mathrm{NMR}\left(\mathrm{CDCl}_{3}, 300 \mathrm{MHz}, \mathrm{rt}\right) \delta 1.04$ (t, $\left.J=7.4 \mathrm{~Hz}, 3 \mathrm{H}\right), 1.10$ (s, $12 \mathrm{H}), 2.47(\mathrm{q}, J=7.4 \mathrm{~Hz}, 2 \mathrm{H}), 5.53(\mathrm{~s}, 1 \mathrm{H}), 7.32(\mathrm{~d}, J=8.1 \mathrm{~Hz}, 2 \mathrm{H}), 7.54(\mathrm{~d}, J=8.1 \mathrm{~Hz}, 2 \mathrm{H}) ;{ }^{13} \mathrm{C} \mathrm{NMR}$ $\left(\mathrm{CDCl}_{3}, 75 \mathrm{MHz}, \mathrm{rt}\right) \delta 12.3,24.5,33.4,83.1,124.3$ (q, $\left.J=270.4 \mathrm{~Hz}\right), 124.4$ (q, $\left.J=3.8 \mathrm{~Hz}\right), 128.2,129.2$, (q, $J$ $=32.0 \mathrm{~Hz}), 143.8,146.9,162.6 ;{ }^{11} \mathrm{~B} \mathrm{NMR}\left(\mathrm{CDCl}_{3}, 96 \mathrm{MHz}, \mathrm{rt}\right) \delta 29.81 ;{ }^{19} \mathrm{~F} \mathrm{NMR}\left(\mathrm{CDCl}_{3}, 282 \mathrm{MHz}, \mathrm{rt}\right)-$ 62.89. MS (EI, m/z (relative intensity)): 326 (M+ 49), 311 (22), 269 (20), 240 (47), 226 (35), 211 (100), 207 (50), 173 (22), 101 (45), 85 (19). Anal. Calcd for $\mathrm{C}_{17} \mathrm{H}_{22} \mathrm{BF}_{3} \mathrm{O}_{2}: \mathrm{C}, 62.60 ; \mathrm{H}, 6.80 \%$. Found: $\mathrm{C}, 62.59$; $\mathrm{H}$, $6.81 \%$.

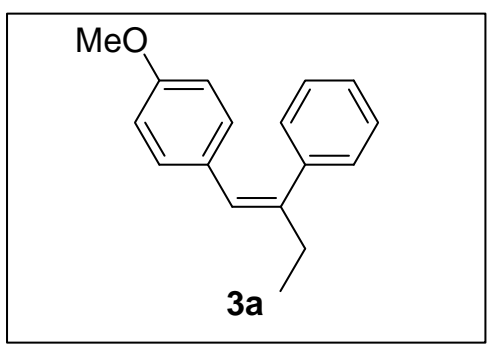

General Procedure for Suzuki-Miyaura Cross-Coupling Reaction of 2a-2c with Aryl Iodides: Formation of (Z)-1-(4-Methoxyphenyl)-2-phenyl-1-butene (3a). $\quad$ To a solution of bis(dibenzylidene)palladium ( $\left.\mathrm{Pd}(\mathrm{dba})_{2}\right)(115 \mathrm{mg}, 0.20 \mathrm{mmol}, 10 \mathrm{~mol} \%), \mathrm{P}^{t} \mathrm{Bu}_{3}(4 \mathrm{~mL}, 0.1 \mathrm{M}$ hexane solution, 
$0.40 \mathrm{mmol}, 20 \mathrm{~mol} \%$ ), and (Z)-4,4,5,5-tetramethyl-2-(2-phenyl-1-buten-1-yl)-1,3,2-dioxaborolane (2a) (516 $\mathrm{mg}, 2.0 \mathrm{mmol})$ in THF $(20 \mathrm{~mL})$ in a $50 \mathrm{~mL}$ of Schlenk tube under an $\mathrm{Ar}$ atmosphere were added 4-iodoanisole $(515 \mathrm{mg}, 2.2 \mathrm{mmol})$ and $\mathrm{KOH}$ aqueous solution $(2 \mathrm{~mL}, 3.0 \mathrm{M}$ solution, $6.0 \mathrm{mmol})$ at room temperature. The reaction mixture was stirred for $12 \mathrm{~h}$ at room temperature, quenched with ammonium chloride, and extracted with diethyl ether $(25 \mathrm{~mL} \times 2)$. The combined ethereal layer was washed with $\mathrm{NaHCO}_{3}$ aq., brine and dried over $\mathrm{MgSO}_{4}$. Filtration and evaporation afforded a brown oil. Bulb to bulb distillation (190 ${ }^{\circ} \mathrm{C} / 2$ Torr) gave $3 a$ (448 mg, $1.62 \mathrm{mmol}, 81 \%$ yield) as a colorless liquid. $R_{f}=0.40$ (hexane). FT-IR (neat, $\mathrm{cm}^{-1}$ ): 2964 (m), 2933 (m), $1608(\mathrm{w}), 1251$ (s), 826 (s), 701 (s). ${ }^{1} \mathrm{H} \mathrm{NMR}\left(\mathrm{CDCl}_{3}, 300 \mathrm{MHz}, \mathrm{rt}\right) \delta 1.09$ (t, $\left.J=7.2 \mathrm{~Hz}, 3 \mathrm{H}\right), 2.52$ (q, $J=$ $7.2 \mathrm{~Hz}, 2 \mathrm{H}), 3.74$ (s, 3H), 6.40 (s, 1H), 6.66 (d, $J=8.1 \mathrm{~Hz}, 2 \mathrm{H}), 6.88$ (d, $J=8.1 \mathrm{~Hz}, 2 \mathrm{H}), 7.18-7.37$ (m, 5H);

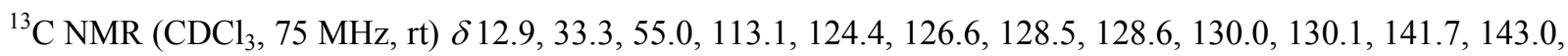
157.8. MS (EI, m/z (relative intensity)): 238 ( $\left.\mathrm{M}^{+}, 100\right), 223$ (57), 208 (10), 178 (12), 165 (19), 145 (21), 129 (10), 121 (19), 115 (43), 91 (18). Anal. Calcd for $\mathrm{C}_{17} \mathrm{H}_{18} \mathrm{O}_{2}$ : C, 85.67; H, 7.61\%. Found: C, 85.60; H, 7.61\%.

Preparation of $\mathbf{3 b} \mathbf{b} \mathbf{3 q}$ were carried out analogously to $\mathbf{3 a .}$

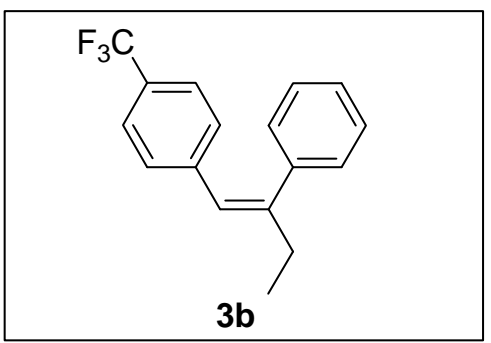

(Z)-1-(4-Trifluoromethylphenyl)-2-phenyl-1-butene (3b). Colorless liquid. Isolated yield was 83\% (459 $\mathrm{mg}, 1.66 \mathrm{mmol}$ ). Bp. $150{ }^{\circ} \mathrm{C} / 2$ Torr. $R_{f}=0.50$ (hexane). FT-IR (neat, $\mathrm{cm}^{-1}$ ): 2970 (m), 2934 (m), 1615 (w), 1123 (m), 759 (s), 701 (s). ${ }^{1} \mathrm{H}$ NMR $\left(\mathrm{CDCl}_{3}, 300 \mathrm{MHz}, \mathrm{rt}\right) \delta 1.10$ (t, $\left.J=7.4 \mathrm{~Hz}, 3 \mathrm{H}\right), 2.55$ (q, $\left.J=7.3 \mathrm{~Hz}, 2 \mathrm{H}\right)$, $6.45(\mathrm{~s}, 1 \mathrm{H}), 7.01(\mathrm{~d}, J=8.1 \mathrm{~Hz}, 2 \mathrm{H}), 7.09(\mathrm{~d}, J=8.1 \mathrm{~Hz}, 2 \mathrm{H}), 7.28-7.36(\mathrm{~m}, 5 \mathrm{H}) ;{ }^{13} \mathrm{C} \mathrm{NMR}\left(\mathrm{CDCl}_{3}, 75 \mathrm{MHz}\right.$, rt) $\delta 12.7,33.6,123.8,124.2(\mathrm{q}, J=270.0 \mathrm{~Hz}), 124.7(\mathrm{q}, J=3.8 \mathrm{~Hz}), 127.2,127.8(\mathrm{q}, J=31.9 \mathrm{~Hz}), 128.3$, 128.6, 129,0, 140.8, 141.2, 147.7; ${ }^{19} \mathrm{~F}$ NMR $\left(\mathrm{CDCl}_{3}, 282 \mathrm{MHz}, \mathrm{rt}\right)-62.95$. MS (EI, m/z (relative intensity)): 276 (M+1 100), 261 (38), 247 (30), 207 (17), 183 (30), 178 (20), 129 (29), 117 (51), 115 (57), 91 (43). Anal. Calcd for $\mathrm{C}_{17} \mathrm{H}_{15} \mathrm{~F}_{3}$ : C, 73.90; H, 5.47\%. Found: C, 73.93; H, 5.55\%. 


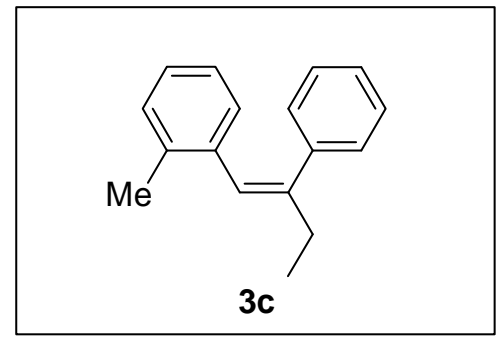

(Z)-1-(2-Methylphenyl)-2-phenyl-1-butene (3c). ${ }^{2}$ Colorless liquid. Isolated yield was 83\% (369 mg, 1.66 mmol). Bp. $180{ }^{\circ} \mathrm{C} / 2$ Torr. $R_{f}=0.70$ (hexane). FT-IR (neat, $\left.\mathrm{cm}^{-1}\right): 2966(\mathrm{~m}), 2932(\mathrm{~m}), 1600(\mathrm{w}), 1459(\mathrm{~m})$, 732 (s), 698 (s). ${ }^{1} \mathrm{H}$ NMR $\left(\mathrm{CDCl}_{3}, 300 \mathrm{MHz}, \mathrm{rt}\right) \delta 1.14(\mathrm{t}, J=7.4 \mathrm{~Hz}, 3 \mathrm{H}), 2.33$ (s, 3H), $2.61(\mathrm{q}, J=7.4 \mathrm{~Hz}$, $2 \mathrm{H}), 6.53(\mathrm{~s}, 1 \mathrm{H}), 6.76(\mathrm{~d}, J=7.4 \mathrm{~Hz}, 1 \mathrm{H}), 6.86(\mathrm{t}, J=7.2 \mathrm{~Hz}, 1 \mathrm{H}), 7.01-7.22(\mathrm{~m}, 7 \mathrm{H}) ;{ }^{13} \mathrm{C} \mathrm{NMR}\left(\mathrm{CDCl}_{3}, 75\right.$ $\mathrm{MHz}, \mathrm{rt}) \delta 13.3,20.0,32.4,124.1,125.1,126.1,126.5,127.9,128.7,129.5,129.8,136.2,137.2,141.0,144.8$. MS (EI, m/z (relative intensity)): $222\left(\mathrm{M}^{+}, 100\right), 207$ (33), 193 (48), 179 (28), 165 (16), 129 (44), 117 (10), 115 (50), 105 (10), $91(27)$.

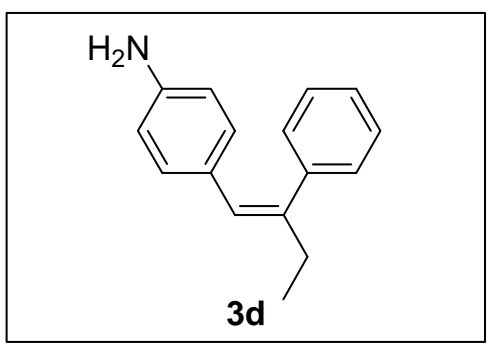

(Z)-1-(4-Aminophenyl)-2-phenyl-1-butene (3d). Colorless liquid. Isolated yield was 80\% (357 mg, 1.60 mmol). Bp. $180^{\circ} \mathrm{C} / 2$ Torr. $R_{f}=0.10$ (hexane/ethyl acetate $\left.=10 / 1\right)$. FT-IR $\left(\mathrm{CHCl}_{3}, \mathrm{~cm}^{-1}\right): 3458(\mathrm{~m}), 3376(\mathrm{~m})$, $2964(\mathrm{~m}), 2930$ (m), 1620 (m), 826 (m), 763 (s), 701 (s). ${ }^{1} \mathrm{H}$ NMR (CDCl $\left., 300 \mathrm{MHz}, \mathrm{rt}\right) \delta 1.05$ (t, J=7.2 Hz, $3 \mathrm{H}), 2.47$ (q, J=7.2 Hz, 2H), $3.56(\mathrm{~s}, 2 \mathrm{H}), 6.32(\mathrm{~s}, 1 \mathrm{H}), 6.48(\mathrm{~d}, J=8.7 \mathrm{~Hz}, 2 \mathrm{H}), 6.74(\mathrm{~d}, J=8.7 \mathrm{~Hz}, 2 \mathrm{H}), 7.16$ $(\mathrm{d}, J=8.1 \mathrm{~Hz}, 2 \mathrm{H}), 7.24-7.33(\mathrm{~m}, 3 \mathrm{H}) ;{ }^{13} \mathrm{C} \mathrm{NMR}\left(\mathrm{CDCl}_{3}, 75 \mathrm{MHz}, \mathrm{rt}\right) \delta 13.0,33.5,114.8,124.7,124.7,126.5$, 128.4, 128.6, 129.9, 141.86, 141.94, 143.8. MS (EI, m/z (relative intensity)): 223 ( $\left.\mathrm{M}^{+}, 100\right), 208$ (66), 193 (22), 165 (13), 130 (43), 115 (69), 106 (55), 96 (12), 91 (41), 77 (17). Anal. Calcd for $\mathrm{C}_{16} \mathrm{H}_{17} \mathrm{~N}: \mathrm{C}, 86.05 ; \mathrm{H}$, 7.67; N, 6.27\%. Found: C, 85.69; H, 7.74; N, 6.15\%.

(2) James, B. G.; Pattenden, G. J. Chem. Soc., Perkin Trans. 1, 1974, 1204-1208. 


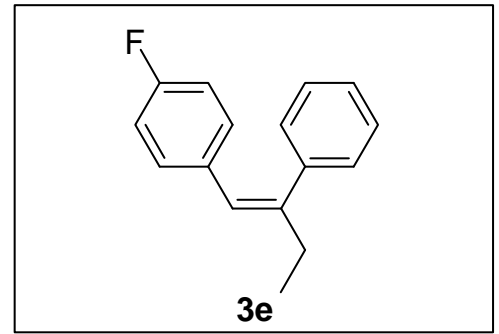

(Z)-1-(4-Fluorophenyl)-2-phenyl-1-butene (3e). Colorless liquid. Isolated yield was 55\% (249 mg, 1.10 mmol). Bp. $140^{\circ} \mathrm{C} / 2$ Torr. $R_{f}=0.40$ (hexane). FT-IR (neat, $\mathrm{cm}^{-1}$ ): $2968(\mathrm{~m}), 2933(\mathrm{~m}), 1602$ (m), 1229 (s), $828(\mathrm{~m}), 762(\mathrm{~s}), 700$ (s). ${ }^{1} \mathrm{H} \mathrm{NMR}\left(\mathrm{CDCl}_{3}, 300 \mathrm{MHz}, \mathrm{rt}\right) \delta 1.09(\mathrm{t}, J=7.5 \mathrm{~Hz}, 3 \mathrm{H}), 2.52(\mathrm{q}, J=7.5 \mathrm{~Hz}, 2 \mathrm{H})$, $6.41(\mathrm{~s}, 1 \mathrm{H}), 6.78(\mathrm{t}, J=7.5 \mathrm{~Hz}, 2 \mathrm{H}), 6.90(\mathrm{t}, J=8.1 \mathrm{~Hz}, 2 \mathrm{H}), 7.15(\mathrm{~d}, J=7.8,2 \mathrm{H}), 7.26-7.35(\mathrm{~m}, 3 \mathrm{H}) ;{ }^{13} \mathrm{C}$ NMR $\left(\mathrm{CDCl}_{3}, 75 \mathrm{MHz}, \mathrm{rt}\right) \delta 12.8,33.4,114.6(\mathrm{~d}, J=21.0 \mathrm{~Hz}), 123.9,127.0,128.5(\mathrm{~d}, J=4.0 \mathrm{~Hz}), 130.4(\mathrm{~d}, J$ $=7.7 \mathrm{~Hz}), 133.6(\mathrm{~d}, J=3.4 \mathrm{~Hz}), 141.2,144.8,159.5,162.7 ;{ }^{19} \mathrm{~F} \mathrm{NMR}\left(\mathrm{CDCl}_{3}, 282 \mathrm{MHz}, \mathrm{rt}\right)-116.75 . \mathrm{MS}(\mathrm{EI}$, m/z (relative intensity)): $226\left(\mathrm{M}^{+}, 100\right), 210(58), 196$ (41), 183 (12), 133 (62), 117 (36), 115 (83), 109 (31), 98 (12), 91 (41). Anal. Calcd for $\mathrm{C}_{16} \mathrm{H}_{15} \mathrm{~F}: \mathrm{C}, 84.92 ; \mathrm{H}, 6.68 \%$. Found: C, 84.95; H, 6.78\%.

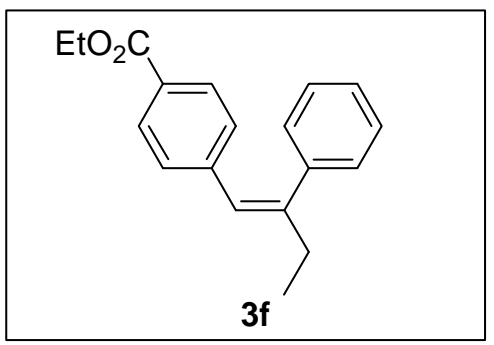

(Z)-1-(4-Ethoxycarbonylphenyl)-2-phenyl-1-butene (3f). Colorless liquid. Isolated yield was $82 \%$ (473 mg, $1.64 \mathrm{mmol}$ ). Bp. $200{ }^{\circ} \mathrm{C} / 2$ Torr. $R_{f}=0.10$ (hexane). FT-IR (neat, $\left.\mathrm{cm}^{-1}\right): 2969(\mathrm{~m}), 2934(\mathrm{~m}), 1717(\mathrm{~s})$, 160 6(w), 1367 (m), $701(\mathrm{~s}) .{ }^{1} \mathrm{H}$ NMR $\left(\mathrm{CDCl}_{3}, 300 \mathrm{MHz}, \mathrm{rt}\right) \delta 1.09$ (t, $\left.J=7.3 \mathrm{~Hz}, 3 \mathrm{H}\right), 1.34$ (t, J=7.2 Hz, 3H), 2.54 (q, $J=7.4 \mathrm{~Hz}, 2 \mathrm{H}), 4.31$ (q, $J=7.2 \mathrm{~Hz}, 2 \mathrm{H}), 6.46(\mathrm{~s}, 1 \mathrm{H}), 6.97$ (d, $J=8.1 \mathrm{~Hz}, 2 \mathrm{H}), 7.11-7.15(\mathrm{~m}$, 2H), 7.26-7.31 (m, 3H), $7.61(\mathrm{~d}, J=8.7 \mathrm{~Hz}, 2 \mathrm{H}) ;{ }^{13} \mathrm{C} \mathrm{NMR}\left(\mathrm{CDCl}_{3}, 75 \mathrm{MHz}, \mathrm{rt}\right) \delta 12.7,14.3,33.6,60.7,124.4$, 127.1, 127.8, 128.3, 128.6, 128.8, 129.1, 141.0, 142.3, 147.6, 166.5. MS (EI, m/z (relative intensity)): 280 (M+1 100), 235 (19), 207 (36), 191 (18), 179 (35), 165 (16), 129 (35), 117 (13), 115 (32), 91 (22). Anal. Calcd for $\mathrm{C}_{19} \mathrm{H}_{20} \mathrm{O}_{2}$ : C, 81.40; H, 7.19\%. Found: C, 81.26; H, 7.19\%. 


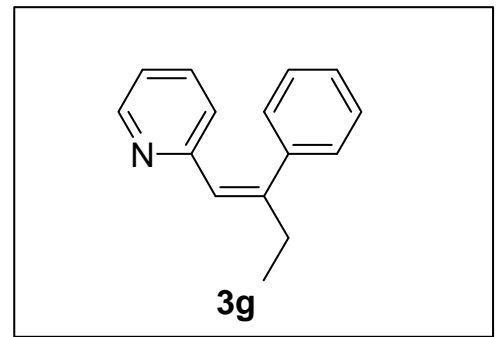

(Z)-1-(2-Pyridyl)-2-phenyl-1-butene (3g). Colorless liquid. Isolated yield was 79\% (331 mg, $1.58 \mathrm{mmol})$. Bp. $140{ }^{\circ} \mathrm{C} / 2$ Torr. $R_{f}=0.10$ (hexane/ethyl acetate =10/1). FT-IR (neat, $\left.\mathrm{cm}^{-1}\right): 2967(\mathrm{~m}), 2932(\mathrm{~m}), 1636(\mathrm{w})$, 1585 (m), 772 (s), 701 (s). ${ }^{1} \mathrm{H}$ NMR $\left(\mathrm{CDCl}_{3}, 300 \mathrm{MHz}, \mathrm{rt}\right) \delta 1.10$ (t, $\left.J=7.5 \mathrm{~Hz}, 3 \mathrm{H}\right), 2.55$ (q, $\left.J=7.2 \mathrm{~Hz}, 2 \mathrm{H}\right)$, $6.58(\mathrm{~d}, J=8.1 \mathrm{~Hz}, 1 \mathrm{H}), 6.63(\mathrm{~s}, 1 \mathrm{H}), 6.94(\mathrm{t}, J=6.9 \mathrm{~Hz}, 1 \mathrm{H}), 7.12-7.15(\mathrm{~m}, 2 \mathrm{H}), 7.20-7.32(\mathrm{~m}, 4 \mathrm{H}), 8.48(\mathrm{~d}, J$ $=7.8 \mathrm{~Hz}, 1 \mathrm{H}) ;{ }^{13} \mathrm{C} \mathrm{NMR}\left(\mathrm{CDCl}_{3}, 75 \mathrm{MHz}, \mathrm{rt}\right) \delta 12.5,33.4,120.8,123.5,125.8,127.2,128.1,128.6,135.4$, 140.9, 148.6, 149.2, 156.5. MS (EI, m/z (relative intensity)): 209 (M+35), 208 (100), 193 (27), 180 (17), 117 (10), 115 (8), 93 (13), 90 (14), 84 (10), 78 (16). HRMS Calcd for $\mathrm{C}_{15} \mathrm{H}_{15} \mathrm{~N}: 209.1204$. Found: $\mathrm{M}^{+}, 209.1216$.

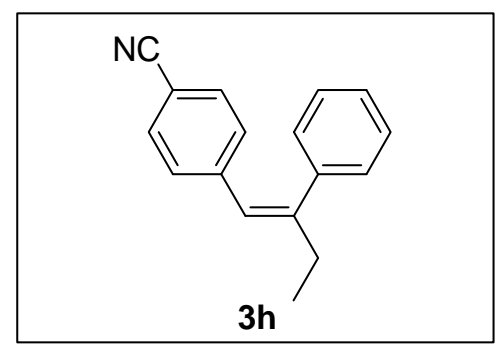

(Z)-1-(4-Cyanophenyl)-2-phenyl-1-butene (3h). Colorless liquid. Isolated yield was 86\% (401 mg, 1.72 mmol). Bp. $170{ }^{\circ} \mathrm{C} / 2$ Torr. $R_{f}=0.10$ (hexane). FT-IR $\left(\mathrm{CHCl}_{3}, \mathrm{~cm}^{-1}\right): 2968(\mathrm{~m}), 2933(\mathrm{~m}), 2224(\mathrm{~s}, \mathrm{vCN})$, $1603(\mathrm{~m}), 828(\mathrm{~m}), 773$ (s), 701 (s). ${ }^{1} \mathrm{H}$ NMR $\left(\mathrm{CDCl}_{3}, 300 \mathrm{MHz}, \mathrm{rt}\right) \delta 1.08$ (t, $\left.J=7.5 \mathrm{~Hz}, 3 \mathrm{H}\right), 2.54$ (q, $J=7.5$ $\mathrm{Hz}, 2 \mathrm{H}), 6.42(\mathrm{~s}, 1 \mathrm{H}), 6.98(\mathrm{~d}, J=8.4 \mathrm{~Hz}, 2 \mathrm{H}), 7.09-7.13(\mathrm{~m}, 2 \mathrm{H}), 7.28-7.36(\mathrm{~m}, 5 \mathrm{H}) ;{ }^{13} \mathrm{C} \mathrm{NMR}\left(\mathrm{CDCl}_{3}, 75\right.$ $\mathrm{MHz}, \mathrm{rt}) \delta 12.6,33.6,109.1,119.1,123.6,127.4,128.1,128.7,129.4,131.5,140.4,142.4,149.1 . \mathrm{MS}$ (EI, m/z (relative intensity)): $233\left(\mathrm{M}^{+}, 100\right), 218$ (63), 204 (48), 190 (11), 155 (16), 140 (40), 117 (43), 115 (45), 91 (27), 77 (12). Anal. Calcd for $\mathrm{C}_{15} \mathrm{H}_{17} \mathrm{~N}$ : C, 87.52; H, 6.48; N, 6.00\%. Found: C, 87.24; H, 6.57; N, 5.93\%. 


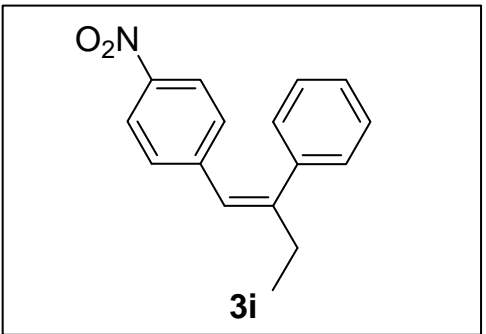

(Z)-1-(4-Nitrophenyl)-2-phenyl-1-butene (3i). Yellow solid. Isolated yield was 92\% (411 mg, 1.84 mmol). Bp. $180^{\circ} \mathrm{C} / 2$ Torr. $R_{f}=0.15$ (hexane). Mp. 30-32 ${ }^{\circ} \mathrm{C}$. FT-IR $\left(\mathrm{CHCl}_{3}, \mathrm{~cm}^{-1}: 2970\right.$ (m), 2934 (m), 1593

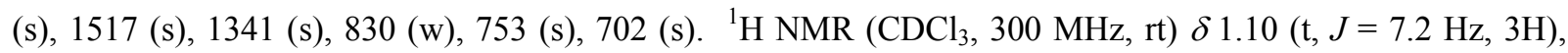
$2.56(\mathrm{q}, J=7.5 \mathrm{~Hz}, 2 \mathrm{H}), 6.48(\mathrm{~s}, 1 \mathrm{H}), 7.03(\mathrm{~d}, J=9.0 \mathrm{~Hz}, 2 \mathrm{H}), 7.12(\mathrm{~d}, J=7.8 \mathrm{~Hz}, 2 \mathrm{H}), 7.29-7.35$ (m, 3H), $7.93(\mathrm{~d}, J=9.0 \mathrm{~Hz}, 2 \mathrm{H}) ;{ }^{13} \mathrm{C} \mathrm{NMR}\left(\mathrm{CDCl}_{3}, 75 \mathrm{MHz}, \mathrm{rt}\right) \delta 12.6,33.6,123.1,123.3,127.6,128.1,128.8,129.4$, 140.3, 144.5, 145.6, 150.0. MS (EI, m/z (relative intensity)): 253 (M+1 100), 238 (17), 223 (14), 207 (28), 192 (41), 178 (37), 93 (46), 165 (22), 152 (11), 129 (10), 115 (31), 91 (25). HRMS Calcd for $\mathrm{C}_{16} \mathrm{H}_{15} \mathrm{NO}_{2}$ : 253.1103. Found: $\mathrm{M}^{+}, 253.1101$.

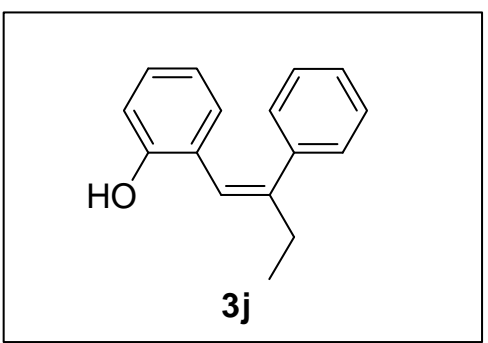

(Z)-1-(2-Hydroxyphenyl)-2-phenyl-1-butene (3j). ${ }^{3}$ White solid. Isolated yield was 58\% (260 mg, 1.16 mmol). Bp. $150{ }^{\circ} \mathrm{C} / 2$ Torr. $R_{f}=0.20$ (hexane/ethyl acetate $\left.=10 / 1\right)$. Mp. 41-43 ${ }^{\circ} \mathrm{C}$. FT-IR $\left(\mathrm{CHCl}_{3}, \mathrm{~cm}^{-1}\right): 3545$ (w), 3420 (w), 2969 (m), 2934 (m), 754 (s), 668 (s). ${ }^{1} \mathrm{H}$ NMR (CDCl $\left.3,300 \mathrm{MHz}, \mathrm{rt}\right) \delta 1.15$ (t, J=7.5 Hz, 3H), $2.66(\mathrm{q}, J=7.5 \mathrm{~Hz}, 2 \mathrm{H}), 5.04(\mathrm{~s}, 1 \mathrm{H}), 6.53(\mathrm{~s}, 1 \mathrm{H}), 6.74(\mathrm{t}, J=7.5 \mathrm{~Hz}, 2 \mathrm{H}), 6.95(\mathrm{~d}, J=7.5 \mathrm{~Hz}, 1 \mathrm{H}), 7.06(\mathrm{~d}, J$ $=7.5 \mathrm{~Hz}, 1 \mathrm{H}), 7.17-7.30(\mathrm{~m}, 5 \mathrm{H}) ;{ }^{13} \mathrm{C} \mathrm{NMR}\left(\mathrm{CDCl}_{3}, 75 \mathrm{MHz}, \mathrm{rt}\right) \delta 13.1,32.2,115.4,119.6,120.2,124.3$, $127.4,128.0,128.2,128.4,130.4,139.9,147.2,152.5$. MS (EI, m/z (relative intensity)): $224\left(\mathrm{M}^{+}, 47\right), 195$ (100), 178 (9), 167 (59), 152 (15), 131 (13), 115 (32), 105 (14), 91 (23), 77 (17). Anal. Calcd for $\mathrm{C}_{16} \mathrm{H}_{16} \mathrm{O}: \mathrm{C}_{\text {, }}$ 85.68; H, 7.19\%. Found: C, 85.36; H, 7.34\%.

(3) Nishimae, S.; Inoue, R.; Shinokubo, H.; Oshima, K. Chem. Lett. 1998, 785-786. 


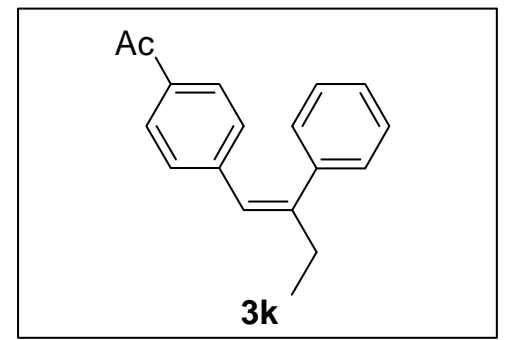

(Z)-1-(4-Acetylphenyl)-2-phenyl-1-butene (3k). Yellow liquid. Isolated yield was 56\% (280 mg, 1.12 mmol). Bp. $190^{\circ} \mathrm{C} / 2$ Torr. $R_{f}=0.10$ (hexane). FT-IR (neat, $\mathrm{cm}^{-1}$ ): $2971(\mathrm{~m}), 2935$ (m), 1684 (s), 1601 (w), 1268 (m), 872 (m), 826 (s), 764 (s), 701 (s). ${ }^{1} \mathrm{H}$ NMR (CDCl $\left.3,300 \mathrm{MHz}, \mathrm{rt}\right) \delta 1.09$ (t, J=7.2 Hz, 3H), 2.50 (s, 3H), $2.54(\mathrm{q}, J=7.5 \mathrm{~Hz}, 2 \mathrm{H}), 6.46(\mathrm{~s}, 1 \mathrm{H}), 6.84(\mathrm{~d}, J=8.4 \mathrm{~Hz}, 2 \mathrm{H}), 7.12-7.15(\mathrm{~m}, 2 \mathrm{H}), 7.29-7.32$ (m, $3 \mathrm{H}), 7.68$ $(\mathrm{d}, J=8.4 \mathrm{~Hz}, 2 \mathrm{H}) ;{ }^{13} \mathrm{C} \mathrm{NMR}\left(\mathrm{CDCl}_{3}, 75 \mathrm{MHz}, \mathrm{rt}\right) \delta 12.7,26.4,33.6,124.2,127.2,127.9,128.3,128.6,128.9$, 134.5, 140.9, 142.6, 148.0, 197.6. MS (EI, m/z (relative intensity)): $250\left(\mathrm{M}^{+}, 100\right), 235$ (68), 207 (14), 191 (13), 178 (29), 165 (18), 129 (25), 115 (18), 110 (11), 91 (20). Anal. Calcd for $\mathrm{C}_{18} \mathrm{H}_{18} \mathrm{O}: \mathrm{C}, 86.36$; H, 7.25\%. Found: C, 86.06; H, 7.23\%.

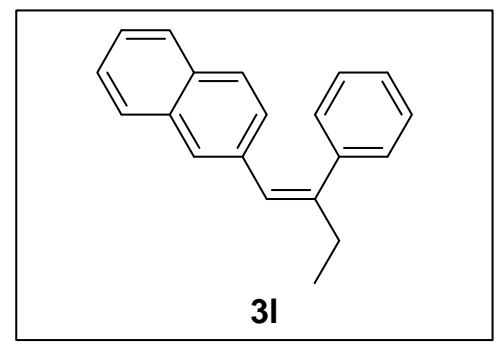

(Z)-1-(1-Naphthyl)-2-phenyl-1-butene (3l). Colorless liquid. Isolated yield was 94\% (486 mg, 1.88 mmol). Bp. $195^{\circ} \mathrm{C} / 2$ Torr. $R_{f}=0.40$ (hexane). FT-IR (neat, $\mathrm{cm}^{-1}$ ): $2965(\mathrm{~m}), 2931(\mathrm{~m}), 1636$ (w), 800 (s), 779 (s), 773 (s), 699 (s). ${ }^{1} \mathrm{H}$ NMR $\left(\mathrm{CDCl}_{3}, 300 \mathrm{MHz}, \mathrm{rt}\right) \delta 1.26(\mathrm{t}, J=7.2 \mathrm{~Hz}, 3 \mathrm{H}), 2.75(\mathrm{q}, J=7.5 \mathrm{~Hz}, 2 \mathrm{H}), 6.98-$ $7.03(\mathrm{~m}, 2 \mathrm{H}), 7.09-7.21(\mathrm{~m}, 6 \mathrm{H}), 7.49-7.56(\mathrm{~m}, 2 \mathrm{H}), 7.65(\mathrm{~d}, J=8.1 \mathrm{~Hz}, 1 \mathrm{H}) 7.86(\mathrm{~d}, J=7.2 \mathrm{~Hz}, 1 \mathrm{H}) 8.21(\mathrm{~d}, J$ $=7.5 \mathrm{~Hz}, 1 \mathrm{H}) ;{ }^{13} \mathrm{C} \mathrm{NMR}\left(\mathrm{CDCl}_{3}, 75 \mathrm{MHz}, \mathrm{rt}\right) \delta 13.3,32.4,123.2,124.7,125.3,125.5,125.7,126.5,126.5$, 127.7, 127.9, 128.4, 128.6, 132.3, 133.4, 135.3, 140.9, 146.5. MS (EI, m/z (relative intensity)): $258\left(\mathrm{M}^{+}, 90\right)$, 243 (24), 229 (100), 215 (9), 202 (7), 165 (44), 152 (10), 141 (12), 115 (28), 91 (34). Anal. Calcd for $\mathrm{C}_{18} \mathrm{H}_{20}$ : C, 92.98; H, 7.02\%. Found: C, 92.88; H, 7.08\%. 


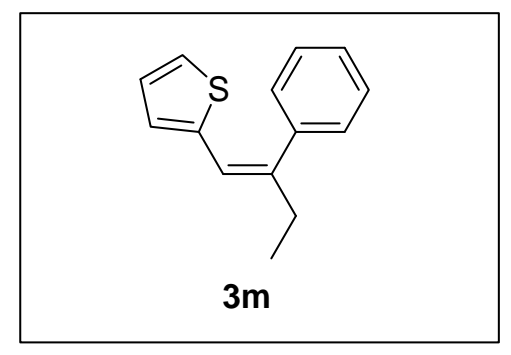

(Z)-1-(2-Thienyl)-2-phenyl-1-butene (3m). Colorless liquid. Isolated yield was 85\% (364 mg, $1.70 \mathrm{mmol}$ ). Bp. $170{ }^{\circ} \mathrm{C} / 2$ Torr. $R_{f}=0.20$ (hexane). FT-IR (neat, $\mathrm{cm}^{-1}$ ): $3050(\mathrm{~m}), 2966(\mathrm{~m}), 2931(\mathrm{~m}), 740$ (s), 699 (s). ${ }^{1} \mathrm{H}$ $\operatorname{NMR}\left(\mathrm{CDCl}_{3}, 300 \mathrm{MHz}, \mathrm{rt}\right) \delta 1.11(\mathrm{t}, J=7.2 \mathrm{~Hz}, 3 \mathrm{H}), 2.48(\mathrm{q}, J=7.4 \mathrm{~Hz}, 2 \mathrm{H}), 6.63(\mathrm{~s}, 1 \mathrm{H}), 6.76(\mathrm{~d}, J=3.5$ $\mathrm{Hz}, 1 \mathrm{H}), 6.81-6.84(\mathrm{~m}, 1 \mathrm{H}), 6.96(\mathrm{~d}, J=3.5 \mathrm{~Hz}, 1 \mathrm{H}), 7.21-7.25(\mathrm{~m}, 2 \mathrm{H}), 7.38-7.47(\mathrm{~m}, 3 \mathrm{H}) ;{ }^{13} \mathrm{C} \mathrm{NMR}\left(\mathrm{CDCl}_{3}\right.$, $75 \mathrm{MHz}, \mathrm{rt}) \delta 12.7,33.6,118.7,124.7,125.9,126.8,127.4,128.7,128.9,141.1,141.1,143.6$. MS (EI, m/z (relative intensity)): $214\left(\mathrm{M}^{+}, 100\right), 199$ (48), 185 (20), 165 (41), 152 (11), 129 (8), 115 (61), 97 (22), 91 (16), 77 (9). Anal. Calcd for $\mathrm{C}_{14} \mathrm{H}_{14} \mathrm{~S}$ : C, 78.46; H, 6.58\%. Found: C, 78.40; H, 6.61\%.

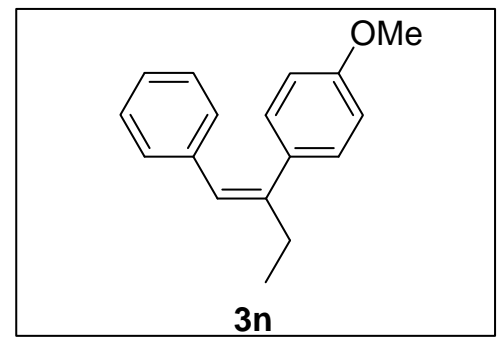

(Z)-1-Phenyl-2-(4-methoxyphenyl)-1-butene (3n). Colorless liquid. Isolated yield was 85\% (405 mg, 1.70 mmol). Bp. $180^{\circ} \mathrm{C} / 2$ Torr. $R_{f}=0.40$ (hexane). FT-IR (neat, $\mathrm{cm}^{-1}$ ): $2964(\mathrm{~m}), 2933(\mathrm{~m}), 1607$ (w), 1245 (m), 832 (s). ${ }^{1} \mathrm{H} \mathrm{NMR}\left(\mathrm{CDCl}_{3}, 300 \mathrm{MHz}, \mathrm{rt}\right) \delta 1.09$ (t, $\left.J=7.2 \mathrm{~Hz}, 3 \mathrm{H}\right), 2.52(\mathrm{q}, J=7.2 \mathrm{~Hz}, 2 \mathrm{H}), 3.83(\mathrm{~s}, 3 \mathrm{H}), 6.43$ $(\mathrm{s}, 1 \mathrm{H}), 6.83-6.87(\mathrm{~m}, 2 \mathrm{H}), 6.88-7.00(\mathrm{~m}, 2 \mathrm{H}), 6.88-7.13(\mathrm{~m}, 5 \mathrm{H}) ;{ }^{13} \mathrm{C} \mathrm{NMR}\left(\mathrm{CDCl}_{3}, 75 \mathrm{MHz}, \mathrm{rt}\right) \delta 12.9,33.5$, $55.1,113.8,124.8,125.9,127.8,128.9,129.6,133.5,137.8,144.4,158.4$. MS (EI, m/z (relative intensity)): 238 (M+, 100), 223 (18), 209 (23), 194 (11), 178 (13), 165 (24), 147 (20), 129 (13), 115 (33), 91 (20). Anal. Calcd for $\mathrm{C}_{17} \mathrm{H}_{18} \mathrm{O}$ : C, 85.67; H, 7.61\%. Found: C, 85.44; H, 7.73\%. 


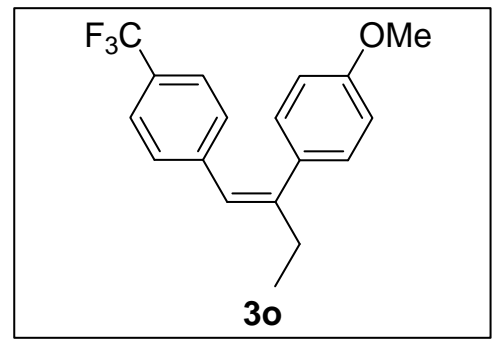

(Z)-1-(4-Trifluoromethylphenyl)-2-(4-methoxypheny)-1-butene (3o). Colorless liquid. Isolated yield was $72 \%$ (441 mg, $1.44 \mathrm{mmol}$ ). Bp. $170{ }^{\circ} \mathrm{C} / 2$ Torr. $R_{f}=0.20$ (hexane). FT-IR (neat, $\left.\mathrm{cm}^{-1}\right): 2968(\mathrm{~m}), 2936(\mathrm{~m})$, $1609(\mathrm{w}), 1246(\mathrm{~m}), 1124(\mathrm{~m}), 833(\mathrm{~s}) .{ }^{1} \mathrm{H} \mathrm{NMR}\left(\mathrm{CDCl}_{3}, 300 \mathrm{MHz}, \mathrm{rt}\right) \delta 1.08(\mathrm{t}, J=7.4 \mathrm{~Hz}, 3 \mathrm{H}), 2.52(\mathrm{q}, J=$ $7.2 \mathrm{~Hz}, 2 \mathrm{H}), 3.82(\mathrm{~s}, 3 \mathrm{H}), 6.42(\mathrm{~s}, 1 \mathrm{H}), 6.84-6.88(\mathrm{~m}, 2 \mathrm{H}), 7.03-7.08(\mathrm{~m}, 4 \mathrm{H}), 7.35(\mathrm{~d}, J=7.4 \mathrm{~Hz}, 2 \mathrm{H}) ;{ }^{13} \mathrm{C}$ $\operatorname{NMR}\left(\mathrm{CDCl}_{3}, 75 \mathrm{MHz}, \mathrm{rt}\right) \delta 12.8,33.5,55.1,114.0,123.5,124.3(\mathrm{q}, J=270.1 \mathrm{~Hz}), 124.7(\mathrm{q}, J=3.8 \mathrm{~Hz})$, $127.6(\mathrm{q}, J=32.2 \mathrm{~Hz}), 130.2,131.8,132.8,141.5,147.2,158.8 ;{ }^{19} \mathrm{~F}$ NMR $\left(\mathrm{CDCl}_{3}, 282 \mathrm{MHz}, \mathrm{rt}\right)-62.91 . \mathrm{MS}$ (EI, m/z (relative intensity)): $306\left(\mathrm{M}^{+}, 100\right), 291$ (12), 277 (24), 262 (11), 183 (14), 178 (8), 165 (14), 147 (26), 121 (8), 108 (16), 91 (11). Anal. Calcd for $\mathrm{C}_{18} \mathrm{H}_{17} \mathrm{~F}_{3} \mathrm{O}$ : C, 70.58; H, 5.59\%. Found: C, 70.63; H, 5.59\%.

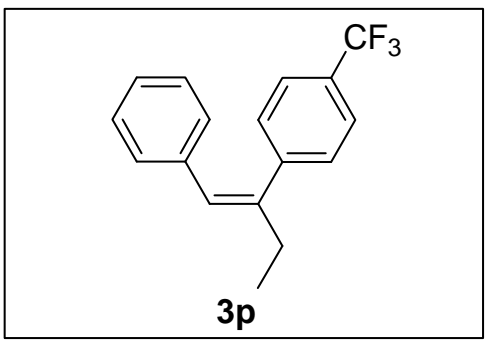

(Z)-1-Phenyl-2-(4-trifluoromethylphenyl)-1-butene (3p). Colorless liquid. Isolated yield was $85 \%$ (470 $\mathrm{mg}, 1.70 \mathrm{mmol}$ ). Bp. $150^{\circ} \mathrm{C} / 2$ Torr. $R_{f}=0.50$ (hexane). FT-IR (neat, $\left.\mathrm{cm}^{-1}\right): 2970(\mathrm{~m}), 2935(\mathrm{~m}), 1615(\mathrm{w})$, $1128(\mathrm{~m}), 756$ (s), 696 (s). ${ }^{1} \mathrm{H} \mathrm{NMR}\left(\mathrm{CDCl}_{3}, 300 \mathrm{MHz}, \mathrm{rt}\right) \delta 1.07$ (t, $\left.J=7.8 \mathrm{~Hz}, 3 \mathrm{H}\right), 2.52(\mathrm{q}, J=7.8 \mathrm{~Hz}, 2 \mathrm{H})$, $6.52(\mathrm{~s}, 1 \mathrm{H}), 6.90-6.91(\mathrm{~m}, 2 \mathrm{H}), 7.09-7.13(\mathrm{~m}, 3 \mathrm{H}), 7.27-7.28(\mathrm{~m}, 2 \mathrm{H}), 7.55(\mathrm{~d}, J=8.1 \mathrm{~Hz}, 2 \mathrm{H}) ;{ }^{13} \mathrm{C} \mathrm{NMR}$ $\left(\mathrm{CDCl}_{3}, 75 \mathrm{MHz}, \mathrm{rt}\right) \delta 12.8,33.1,124.3(\mathrm{q}, J=270.4 \mathrm{~Hz}), 125.4(\mathrm{q}, J=3.7 \mathrm{~Hz}), 126.3,126.4,128.0,128.9$ (q, $J=32.0 \mathrm{~Hz}), 129.0,129.1,136.9,143.3,145.4 ;{ }^{19} \mathrm{~F} \mathrm{NMR}\left(\mathrm{CDCl}_{3}, 282 \mathrm{MHz}, \mathrm{rt}\right)-62.90 . \mathrm{MS}$ (EI, m/z (relative intensity)): 276 (M+, 100), 261 (52), 247 (22), 207 (16), 183 (29), 178 (17), 165 (15), 129 (33), 115 (85), 105 (18), 91 (96). Anal. Calcd for $\mathrm{C}_{17} \mathrm{H}_{15} \mathrm{~F}_{3}$ : C, 73.90; H, 5.47\%. Found: C, 74.06; H, 5.62\%. 


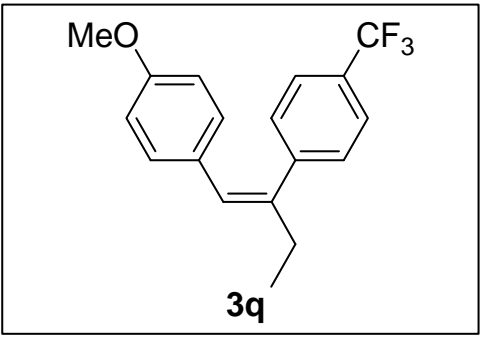

(Z)-1-(4-Methoxypheny)-2-(4-trifluoromethylphenyl)-1-butene (3q). Colorless liquid. Isolated yield was $75 \%$ (459.5 mg, $1.50 \mathrm{mmol}$ ). Bp. $170{ }^{\circ} \mathrm{C} / 2$ Torr. $R_{f}=0.20$ (hexane). FT-IR (neat, $\left.\mathrm{cm}^{-1}\right): 2967(\mathrm{~m}), 2935(\mathrm{~m})$, $1608(\mathrm{w}), 1253(\mathrm{~m}), 1126(\mathrm{~m}), 827(\mathrm{~s}) .{ }^{1} \mathrm{H} \mathrm{NMR}\left(\mathrm{CDCl}_{3}, 300 \mathrm{MHz}, \mathrm{rt}\right) \delta 1.05(\mathrm{t}, J=7.8 \mathrm{~Hz}, 3 \mathrm{H}), 2.50(\mathrm{q}, J=$ $7.8 \mathrm{~Hz}, 2 \mathrm{H}), 3.74(\mathrm{~s}, 3 \mathrm{H}), 6.44(\mathrm{~s}, 1 \mathrm{H}), 6.65(\mathrm{~d}, J=7.8 \mathrm{~Hz}, 2 \mathrm{H}), 6.83(\mathrm{~d}, J=8.4 \mathrm{~Hz}, 2 \mathrm{H}), 7.27-7.28(\mathrm{~m}, 2 \mathrm{H})$, $7.55(\mathrm{~d}, J=7.8 \mathrm{~Hz}, 2 \mathrm{H}) ;{ }^{13} \mathrm{C} \mathrm{NMR}\left(\mathrm{CDCl}_{3}, 75 \mathrm{MHz}, \mathrm{rt}\right) \delta 12.9,33.1,55.1,113.4,124.3(\mathrm{q}, J=270.3 \mathrm{~Hz})$, $125.4(\mathrm{q}, J=3.7 \mathrm{~Hz}), 126.8,128.3$ (q, $J=32.5 \mathrm{~Hz}), 129.1,129.5,130.1,141.4,145.6,158.1 ;{ }^{19} \mathrm{~F} \mathrm{NMR}\left(\mathrm{CDCl}_{3}\right.$, $282 \mathrm{MHz}, \mathrm{rt})$-62.86. MS (EI, m/z (relative intensity)): $306\left(\mathrm{M}^{+}, 100\right), 291$ (68), $276(8), 251$ (7), 183 (28), 178 (10), 165 (10), 145 (13), 121 (56), 115 (14), 108 (12), 91 (19). Anal. Calcd for $\mathrm{C}_{18} \mathrm{H}_{17} \mathrm{~F}_{3} \mathrm{O}$ : C, 70.58; H, 5.59\%. Found: C, 70.72; H, 5.66\%.

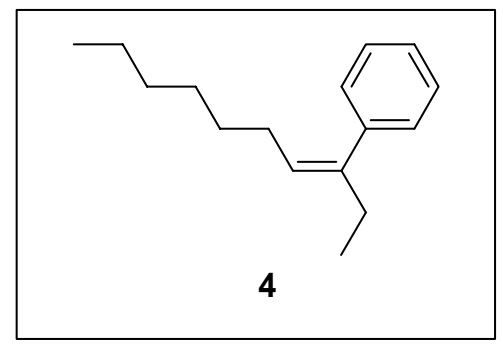

[(1Z)-1-Ethyl-1-octenyl]benzene (4). Under an $\mathrm{Ar}$ atmosphere, $\mathrm{Ni}(\mathrm{cod})_{2}(22.4 \mathrm{mg}, 0.080 \mathrm{mmol})$, bathophenanthroline $(53 \mathrm{mg}, \quad 0.16 \mathrm{mmol}), \quad(Z)-4,4,5,5$-tetramethyl-2-(2-phenyl-1-buten-1-yl)-1,3,2dioxaborolane (2a) $(619.6 \mathrm{mg}, 2.4 \mathrm{mmol})$, and $\mathrm{KO}^{t} \mathrm{Bu}(360 \mathrm{mg}, 3.2 \mathrm{mmol})$ were placed in a $50 \mathrm{~mL} \mathrm{Schlenk}$ tube equipped with a stirring bar. Then 2-butanol $(12 \mathrm{~mL})$ as a solvent was added and the reaction mixture was stirred for 10 minutes at room temperature. To the resulting dark purple mixture, 1-iodohexane $(295 \mu \mathrm{L}, 2.0$ mmol) was then added by syringe, and the reaction mixture was heated at $60{ }^{\circ} \mathrm{C}$ (oil bath) for $5 \mathrm{~h}$. The resulting white suspension was allowed to cool to room temperature, and then it was filtered through a short pad of silica gel. The filtrate was concentrated under vacuum, and the product was isolated by bulb to bulb distillation $\left(120^{\circ} \mathrm{C} / 2\right.$ Torr) as a colorless oil. Isolated yield was $44 \%$ (190 mg, $\left.0.88 \mathrm{mmol}\right)$. Bp. $120^{\circ} \mathrm{C} / 2 \mathrm{Torr}$. 
$R_{f}=0.60$ (hexane). FT-IR (neat, $\mathrm{cm}^{-1}$ ): 2960 (m), 2925 (m), 2855 (m), 2360 (m), 2337 (m), $1461(\mathrm{~m}), 1456$ (m), 767 (s), $700(\mathrm{~s}) .{ }^{1} \mathrm{H}$ NMR $\left(\mathrm{CDCl}_{3}, 300 \mathrm{MHz}, \mathrm{rt}\right) \delta 0.86(\mathrm{t}, J=7.2 \mathrm{~Hz}, 3 \mathrm{H}), 0.96$ (t, $\left.J=7.2 \mathrm{~Hz}, 3 \mathrm{H}\right), 1.17-$ 1.34 (m, 8H), 1.91 (q, $J=7.2 \mathrm{~Hz}, 2 \mathrm{H}), 2.34$ (q, $J=7.2 \mathrm{~Hz}, 2 \mathrm{H}), 5.43$ (t, $J=7.5 \mathrm{~Hz}, 1 \mathrm{H}), 7.11-7.15(\mathrm{~m}, 2 \mathrm{H})$,

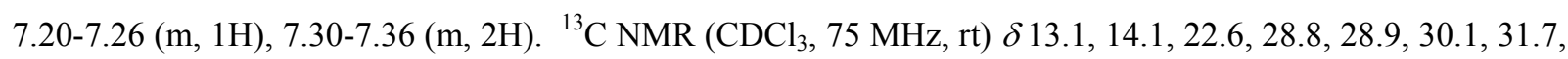
32.1, 126.1, 126.2, 127.9, 128.4, 141.7, 142.4. MS (EI, m/z (relative intensity)): $216\left(\mathrm{M}^{+}, 11\right), 187$ (17), 145 (48), 132 (32), 129 (14), 117 (100), 115 (28), 105 (28), 91 (72), 77 (7). HRMS Calcd for $\mathrm{C}_{16} \mathrm{H}_{24}$ : 216.1878. Found: $\mathrm{M}^{+}, 216.1879$. Synthesis of $(E)$-isomer has been reported. ${ }^{4}$

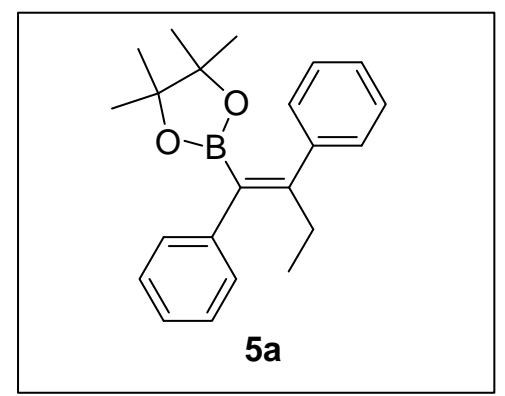

2-[(1E)-1,2-Diphenyl-1-butenyl]-4,4,5,5-tetramethyl-1,3,2-dioxaborolane (5a). [865999-25-3] ${ }^{5}$ To a solution zirconocene dichloride $(1.05 \mathrm{~g}, 3.6 \mathrm{mmol})$ in THF $(18 \mathrm{~mL})$ in a $25 \mathrm{~mL}$ of Schlenk tube under an Ar atmosphere were added dropwise $\mathrm{n}$-BuLi $\left(4.5 \mathrm{~mL}, 7.2 \mathrm{mmol}, 1.6 \mathrm{M}\right.$ hexane solution) at $-78{ }^{\circ} \mathrm{C}$. After the reaction mixture was stirred for $1 \mathrm{~h}$ at $-78^{\circ} \mathrm{C}$, atmospheric ethylene gas was introduced into the vessel for $1 \mathrm{~h}$ at $-78{ }^{\circ} \mathrm{C}$. The reaction mixture was warmed to room temperature and 4,4,5,5-tetramethyl-2-(phenylethynyl)1,3,2-dioxaborolane (1a; $682 \mathrm{mg}, 3 \mathrm{mmol})$ was added. After $1 \mathrm{~h},{ }^{i} \operatorname{PrOH}(185 \mu \mathrm{L}, 2.4 \mathrm{mmol})$ was added and the reaction mixture was stirred for additional $1 \mathrm{~h}$. To the mixture were added $\mathrm{Pd}\left(\mathrm{PPh}_{3}\right)_{4}(336 \mathrm{mg}, 0.3 \mathrm{mmol}), \mathrm{PhI}$ ( $330 \mu \mathrm{L}, 3 \mathrm{mmol}), \mathrm{CuCl}(300 \mathrm{mg}, 3 \mathrm{mmol})$. The mixture was stirred for $1 \mathrm{~h}$ at room temperature and quenched with $1 \mathrm{M}$ hydrochloric acid $(10 \mathrm{~mL})$, and extracted with diethyl ether $(10 \mathrm{~mL} \times 2)$. The combined ethereal layer was washed with brine and dried over $\mathrm{MgSO}_{4}$. Filtration, evaporation, and column chromatography on silica gel $\left(R_{f}=0.2\right.$; hexane $\left./ \mathrm{AcOEt}=19: 1\right)$ gave $5 \mathbf{a}(431 \mathrm{mg}, 1.4 \mathrm{mmol}, 47 \%$ yield $)$ as yellow solid. Mp. 37-38 ${ }^{\circ} \mathrm{C}$. FT-IR (KBr, $\left.\mathrm{cm}^{-1}\right): 2977$ (m), 2932 (m), 2857 (m), 1598 (m), 1492 (m), 1356 (m), 1299 (m), 1269 (m),

(4) Shi, J.-c.; Negishi, E.-i. J. Organomet. Chem. 2003, 687, 518-524.

(5) Shimizu, M,; Nakamaki, C,; Shimono, K,; Schelper, M,; Kurahashi, T,; Hiyama, T. J. Am. Chem. Soc.,2005, 127, 12506-12507. 
$1214(\mathrm{~m}), 1104$ (m), 975 (s), 904 (s), 854 (s), 702 (s). ${ }^{1} \mathrm{H}$ NMR (300 MHz, $\left.\mathrm{CDCl}_{3}, \mathrm{rt}\right) \delta 0.87$ (t, $\left.J=7.5 \mathrm{~Hz}, 3 \mathrm{H}\right)$, 1.00 (s, 12H), 2.43 (q, $J=7.5 \mathrm{~Hz}, 2 \mathrm{H}), 7.23-7.35(\mathrm{~m}, 10 \mathrm{H}) ;{ }^{13} \mathrm{C} \mathrm{NMR}\left(75 \mathrm{MHz}, \mathrm{CDCl}_{3}, \mathrm{rt}\right) \delta 13.6,24.3,27.1$, $83.3,125.9,127.0,127.9,128.1,128.3,128.4,141.4,143.6,153.8$. The carbon signal attached to B was not observed due to low intensity; ${ }^{11} \mathrm{~B} \mathrm{NMR}\left(\mathrm{CDCl}_{3}, 96 \mathrm{MHz}, \mathrm{rt}\right) \delta 30.16$. MS (EI, m/z (relative intensity)): 334 $\left(\mathrm{M}^{+}, 70\right), 277$ (100), 234 (51), $218(32), 130$ (78).

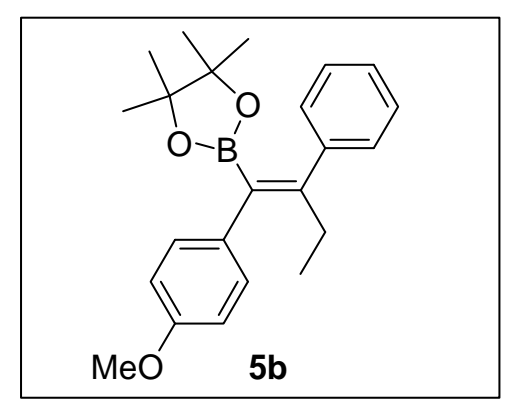

2-[(1E)-1-(4-Methoxyphenyl)-2-phenyl-1-butenyl]-4,4,5,5-tetramethyl-1,3,2-dioxaborolane

(5b).

$[865999-30-0]^{5}$ White solid. Isolated yield was $62 \%(677 \mathrm{mg}, 1.86 \mathrm{mmol}) . R_{f}=0.40$ (hexane/AcOEt $\left.=9: 1\right)$. Mp. 63-64 ${ }^{\circ} \mathrm{C}$. FT-IR (KBr, cm $\left.{ }^{-1}\right): 2972$ (m), 2830 (m), 2874 (m), 1604 (w), 1509 (m), 1462 (m), 1440 (m), 1353 (m), 1283 (m), 1245 (m), 1142 (m), 1033 (s), 854 (s), 703 (s). ${ }^{1} \mathrm{H}$ NMR (300 MHz, $\left.\mathrm{CDCl}_{3}, \mathrm{rt}\right) \delta 0.86$ (t, $J$ $=7.5 \mathrm{~Hz}, 3 \mathrm{H}), 1.00(\mathrm{~s}, 12 \mathrm{H}), 2.44$ (q, $J=7.5 \mathrm{~Hz}, 2 \mathrm{H}), 3.82(\mathrm{~s}, 3 \mathrm{H}), 6.89(\mathrm{~d}, J=8.7 \mathrm{~Hz}, 2 \mathrm{H}), 7.16(\mathrm{~d}, J=8.7 \mathrm{~Hz}$, 2H), 7.27-7.36 (m, 5H); ${ }^{13} \mathrm{C}$ NMR (75 MHz, $\left.\mathrm{CDCl}_{3}, \mathrm{rt}\right) \delta 13.3,24.4,27.0,55.1,83.2,113.6,126.9,127.8$, $128.4,129.5,133.8,143.7,153.4,157.8$. The carbon signal attached to $\mathrm{B}$ was not observed due to low intensity; ${ }^{11} \mathrm{~B} \mathrm{NMR}\left(\mathrm{CDCl}_{3}, 96 \mathrm{MHz}, \mathrm{rt}\right) \delta 30.01 . \mathrm{MS}$ (EI, m/z (relative intensity)): 364 ( $\left.\mathrm{M}^{+}, 100\right), 307$ (35), 264 (33), 249 (35), 160 (46).

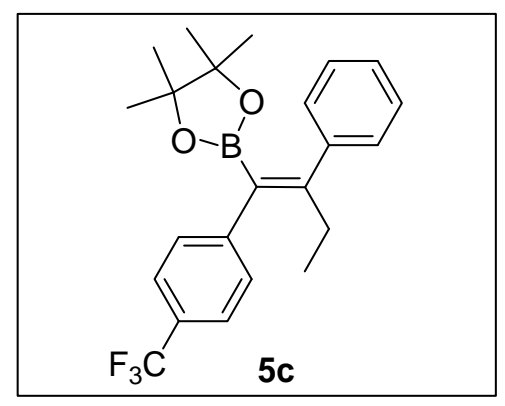

4,4,5,5-Tetramethyl-2-[(1E)-2-phenyl-1-[4-(trifluoromethyl)phenyl]-1-butenyl]-1,3,2-dioxaborolane

(5c). $[865999-27-5]^{5}$ White solid. Isolated yield was $40 \%(481 \mathrm{mg}, 1.20 \mathrm{mmol}) . R_{f}=0.45$ (hexane $/ \mathrm{AcOEt}=$ 
9:1). Mp. $77-79^{\circ} \mathrm{C} . \quad$ FT-IR $\left(\mathrm{KBr}, \mathrm{cm}^{-1}\right): 2978$ (m), 2932 (m), 2874 (m), 1613 (w), 1351 (m), 1325 (m), 1145 (m), $1105(\mathrm{~m}), 1066(\mathrm{~m}), 856(\mathrm{~s}), 701(\mathrm{~s}) .{ }^{1} \mathrm{H} \mathrm{NMR}\left(300 \mathrm{MHz}, \mathrm{CDCl}_{3}, \mathrm{rt}\right) \delta 0.85(\mathrm{t}, J=7.5 \mathrm{~Hz}, 3 \mathrm{H}), 1.00(\mathrm{~s}$, $12 \mathrm{H}), 2.38$ (q, $J=7.5 \mathrm{~Hz} 2 \mathrm{H}), 7.28-7.36(\mathrm{~m}, 7 \mathrm{H}), 7.60(\mathrm{~d}, J=8.1 \mathrm{~Hz}, 2 \mathrm{H}) ;{ }^{13} \mathrm{C} \mathrm{NMR}\left(75 \mathrm{MHz}, \mathrm{CDCl}_{3}, \mathrm{rt}\right)$ $\delta 13.1,24.3,27.3,83.5,124.4(\mathrm{q}, J=270.1 \mathrm{~Hz}), 125.1$ (q, $J=3.7 \mathrm{~Hz}), 127.3,127.9,128.0(\mathrm{q}, J=32.0 \mathrm{~Hz})$, 128.3, 128.7, 143.1, 145.4, 155.4. The carbon signal attached to B was not observed due to low intensity; ${ }^{11} \mathrm{~B}$ NMR $\left(\mathrm{CDCl}_{3}, 96 \mathrm{MHz}, \mathrm{rt}\right) \delta 30.07 ;{ }^{19} \mathrm{~F} \mathrm{NMR}\left(\mathrm{CDCl}_{3}, 282 \mathrm{MHz}, \mathrm{rt}\right) \delta-62.74 . \quad \mathrm{MS}$ (EI, m/z (relative intensity)): $402\left(\mathrm{M}^{+}, 27\right), 345$ (73), 302 (20), 286 (17), 101 (100).

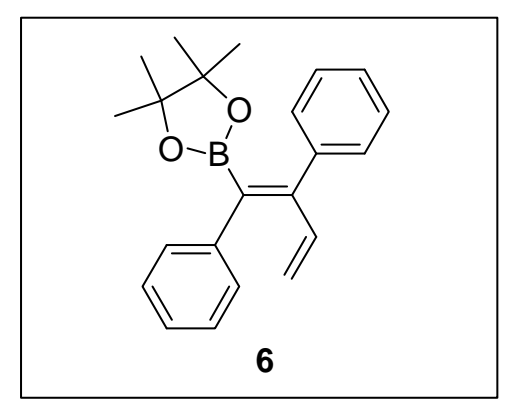

2-[(1E)-1,2-Diphenyl-1,3-butadienyl]-4,4,5,5-tetramethyl-1,3,2-dioxaborolane (6). A $50 \mathrm{~mL}$ Schlenk tube under argon was charged with $\mathrm{Cp}_{2} \mathrm{ZrCl}_{2}(1.05 \mathrm{~g}, 3.6 \mathrm{mmol})$ and $18 \mathrm{~mL}$ of THF. The mixture was cooled to $-78^{\circ} \mathrm{C}$, and then 2 equiv of n-BuLi ( $4.5 \mathrm{~mL}, 7.2 \mathrm{mmol}, 1.6 \mathrm{M}$ THF solution) was added dropwise via syringe. After the reaction mixture was stirred at $-78^{\circ} \mathrm{C}$ for $1 \mathrm{~h}$ under argon, ethylene gas was introduced for $1 \mathrm{~h}$, and $\mathbf{1 a}$ (682 mg, $3 \mathrm{mmol}$ ) was added at $-78^{\circ} \mathrm{C}$. The resulting mixture was gradually warmed to room temperature and stirred for $1 \mathrm{~h}$. To this was added vinyl ethyl ether $(0.640 \mathrm{~mL}, 4.5 \mathrm{mmol})$ at room temperature. After the mixture was stirred for $20 \mathrm{~h}$ at $50{ }^{\circ} \mathrm{C} . \mathrm{CuCl}(300 \mathrm{mg}, 3 \mathrm{mmol}), \mathrm{DMPU}(0.544 \mathrm{~mL}, 4.5 \mathrm{mmol}), \mathrm{Pd}\left(\mathrm{PPh}_{3}\right)_{4}(336$ $\mathrm{mg}, 0.3 \mathrm{mmol}$,), and iodobenzene $(0.369 \mathrm{~mL}, 3.3 \mathrm{mmol})$ was added successively at room temperature, and the mixture was stirred for $3 \mathrm{~h}$ at $50{ }^{\circ} \mathrm{C}$ The reaction mixture was quenched with $3 \mathrm{M} \mathrm{HCl}$, extracted two times with diethyl ether. Extract was dried over $\mathrm{MgSO}_{4}$ and concentrated. Purification with column chromatography on silica gel $\left(R_{f}=0.2\right.$; hexane/ $\left.\mathrm{Et}_{2} \mathrm{O}=19: 1\right)$ afforded the title compound as colorless liquid. Isolated yield was 26\% (259 mg, $0.78 \mathrm{mmol})$. FT-IR (KBr, cm $\left.{ }^{-1}\right): 2978$ (m), 2930 (m), 1559 (w), 1492 (m), 1442 (m), 1362 (m), 1306 (m), 1268 (m), 1215 (m), 1141 (m), 972 (m), 911 (s), 854 (s), 701 (s). ${ }^{1} \mathrm{H}$ NMR (300 MHz, CDCl 3 , rt) $\delta 0.98(\mathrm{~s}, 12 \mathrm{H}), 4.87(\mathrm{~d}, J=18.9 \mathrm{~Hz} 1 \mathrm{H}), 5.17$ (d, $J=12.3 \mathrm{~Hz} 1 \mathrm{H}), 6.74$ (q, $J=9.1 \mathrm{~Hz} 1 \mathrm{H}), 7.25-7.36(\mathrm{~m}, 10 \mathrm{H})$; ${ }^{13} \mathrm{C}$ NMR $\left(75 \mathrm{MHz}, \mathrm{CDCl}_{3}, \mathrm{rt}\right) \delta 24.3,83.4,119.4,126.4,127.2,127.7,128.0,129.2,130.1,136.3,140.1$; $141.1,148.5$. The carbon signal attached to $\mathrm{B}$ was not observed due to their low intensity; ${ }^{11} \mathrm{~B} \mathrm{NMR}\left(\mathrm{CDCl}_{3}\right.$, 
$96 \mathrm{MHz}, \mathrm{rt}) \delta 30.09 . \mathrm{MS}$ (EI, m/z (relative intensity)): $332\left(\mathrm{M}^{+}, 21\right), 275$ (17), 232 (41), 213 (41), 207 (77), 204 (100), 84 (68). HRMS Calcd for $\mathrm{C}_{22} \mathrm{H}_{25} \mathrm{BO}_{2}$ : 332.1948. Found: $\mathrm{M}^{+}, 332.1945$.

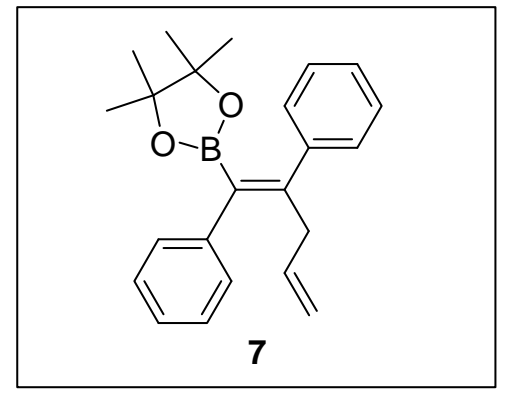

2-[(1E)-1,2-Diphenyl-1,4-pentadienyl]-4,4,5,5-tetramethyl-1,3,2-dioxaborolane (7). A $50 \mathrm{~mL}$ Schlenk tube under argon was charged with $\mathrm{Cp}_{2} \mathrm{ZrCl}_{2}(1.05 \mathrm{~g}, 3.6 \mathrm{mmol})$ and $18 \mathrm{~mL}$ of THF. The mixture was cooled to $-78{ }^{\circ} \mathrm{C}$, and then 2 equiv of n-BuLi (4.5 mL, 7.2 mmol, 1.6 M THF solution) was added dropwise via syringe. After the reaction mixture was stirred at $-78^{\circ} \mathrm{C}$ for $1 \mathrm{~h}$ under argon, ethylene gas ( $1 \mathrm{~atm}$ ) was introduced for $1 \mathrm{~h}$, and 1a $(682 \mathrm{mg}, 3 \mathrm{mmol})$ was added at $-78{ }^{\circ} \mathrm{C}$. The resulting mixture was gradually warmed to room temperature and stirred for $1 \mathrm{~h}$. To this was added allyloxytrimethylsilane $(0.743 \mathrm{~mL}, 4.5 \mathrm{mmol})$ at room temperature. After the mixture was stirred for $20 \mathrm{~h}$ at $50^{\circ} \mathrm{C}, \mathrm{CuCl}(300 \mathrm{mg}, 3 \mathrm{mmol})$, DMPU (0.544 mL, 4.5 $\mathrm{mmol}), \mathrm{Pd}\left(\mathrm{PPh}_{3}\right)_{4}(336 \mathrm{mg}, 0.3 \mathrm{mmol}$,$) , and iodobenzene (0.369 \mathrm{~mL}, 3.3 \mathrm{mmol})$ was added successively at room temperature, and the mixture was stirred for $3 \mathrm{~h}$ at $50{ }^{\circ} \mathrm{C}$. The reaction mixture was quenched with $3 \mathrm{M}$ $\mathrm{HCl}$, extracted two times with diethyl ether. Extract was dried over $\mathrm{MgSO}_{4}$ and concentrated. Purification with column chromatography on silica gel $\left(R_{f}=0.2\right.$; hexane/ $\left.\mathrm{Et}_{2} \mathrm{O}=19: 1\right)$ afforded the title compound as white solid. Isolated yield was $43 \%$ (450 mg, $1.3 \mathrm{mmol}) . \quad R_{f}=0.20$ (hexane $\left./ \mathrm{Et}_{2} \mathrm{O}=19: 1\right)$. Mp. 50-52 ${ }^{\circ} \mathrm{C}$. FT-IR (KBr, cm$\left.{ }^{-1}\right): 2976$ (m), 2927 (m), 1596 (w), 1493 (m), 1443 (m), 1355 (m), 1302 (m), 1265 (m), 1219 (m), 1141 (m), 978 (m), 906 (s), 854 (s), 701 (s). ${ }^{1} \mathrm{H}$ NMR (300 MHz, $\left.\mathrm{CDCl}_{3}, \mathrm{rt}\right) \delta 1.02$ (s, 12H), 3.18 (m, 2H), 4.88 $(\mathrm{m}, 2 \mathrm{H}), 5.63(\mathrm{~m}, 1 \mathrm{H}), 7.21-7.40(\mathrm{~m}, 10 \mathrm{H}) ;{ }^{13} \mathrm{C} \mathrm{NMR}\left(75 \mathrm{MHz}, \mathrm{CDCl}_{3}, \mathrm{rt}\right) \delta 24.3,38.6,83.4,115.9,126.1$, $127.2,127.8,128.1,128.3,128.4,135.8,141.2 ; 143.5,149.2$. The carbon signal attached to B was not observed due to their low intensity; ${ }^{11} \mathrm{~B} \mathrm{NMR}\left(\mathrm{CDCl}_{3}, 96 \mathrm{MHz}, \mathrm{rt}\right) \delta 30.24$. MS (EI, m/z (relative intensity)): 346 ( $\left.\mathrm{M}^{+}, 11\right), 247$ (18), 246 (100), 218 (39), 207 (21), 142 (23), 84 (16). Anal. Calcd for $\mathrm{C}_{23} \mathrm{H}_{27} \mathrm{BO}_{2}$ : C, 79.78 ; H, 7.86\%. Found: C, 79.54; H, 7.89\%. 


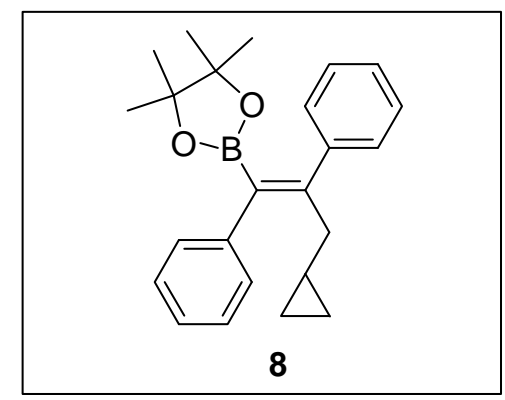

2-[(1E)-1,2-Diphenyl-3-cyclopropyl-1-propenyl]-4,4,5,5-tetramethyl-1,3,2-dioxaborolane (8). A $50 \mathrm{~mL}$ Schlenk tube under argon was charged with $\mathrm{Cp}_{2} \mathrm{ZrCl}_{2}(1.05 \mathrm{~g}, 3.6 \mathrm{mmol})$ and $18 \mathrm{~mL}$ of THF. The mixture was cooled to $-78{ }^{\circ} \mathrm{C}$, and then 2 equiv of $\mathrm{n}-\mathrm{BuLi}(4.5 \mathrm{~mL}, 7.2 \mathrm{mmol}, 1.6 \mathrm{M}$ THF solution) was added dropwise via syringe. After the reaction mixture was stirred at $-78{ }^{\circ} \mathrm{C}$ for $1 \mathrm{~h}$ under argon, ethylene gas $(1 \mathrm{~atm})$ was introduced for $1 \mathrm{~h}$, and $1 \mathrm{a}(682 \mathrm{mg}, 3 \mathrm{mmol})$ was added at $-78^{\circ} \mathrm{C}$. The resulting mixture was gradually warmed to room temperature and stirred for $1 \mathrm{~h}$. To this was added 4-bromo-1-butene $(0.456 \mathrm{~mL}, 4.5 \mathrm{mmol})$ at room temperature. After the mixture was stirred for $20 \mathrm{~h}$ at $50^{\circ} \mathrm{C}, \mathrm{CuCl}(300 \mathrm{mg}, 3 \mathrm{mmol}), \mathrm{DMPU}(0.544 \mathrm{~mL}, 4.5$ mmol), $\mathrm{Pd}\left(\mathrm{PPh}_{3}\right)_{4}(336 \mathrm{mg}, 0.3 \mathrm{mmol})$, and iodobenzene $(0.369 \mathrm{~mL}, 3.3 \mathrm{mmol})$ was added successively at room temperature, and the mixture was stirred for $3 \mathrm{~h}$ at $50^{\circ} \mathrm{C}$. The reaction mixture was quenched with $3 \mathrm{M}$ $\mathrm{HCl}$, extracted two times with diethyl ether. Extract was dried over $\mathrm{MgSO}_{4}$ and concentrated. Purification with column chromatography on silica gel $\left(R_{f}=0.35\right.$; hexane/Et $\left.2 \mathrm{O}=9: 1\right)$ afforded the title compound. Isolated yield was 54\% (584 mg, $1.62 \mathrm{mmol})$. White solid. Isolated yield was 54\% (584mg, $1.62 \mathrm{mmol}) . \quad R_{f}=0.35$ (hexane:Et $\left.{ }_{2} \mathrm{O}=9: 1\right)$. Mp. 56-58 ${ }^{\circ} \mathrm{C}$. FT-IR $\left(\mathrm{KBr}, \mathrm{cm}^{-1}\right): 3003(\mathrm{~m}), 2978(\mathrm{~m}), 1599(\mathrm{w}), 1386(\mathrm{~m}), 1370(\mathrm{~m})$, 1356 (m), 1303 (m), 1264 (m), 1212 (m), 1143 (m), 1020 (m), 969 (m), 855 (s), 764 (s), 705 (s). ${ }^{1} \mathrm{H}$ NMR (300 $\left.\mathrm{MHz} \mathrm{CDCl}_{3}, \mathrm{rt}\right) \delta-0.20(\mathrm{q}, J=5 \mathrm{~Hz}, 2 \mathrm{H}), 0.23(\mathrm{dt}, J=7.5,7.5 \mathrm{~Hz}, 2 \mathrm{H}), 0.56$ (quin, $\left.J=13.5 \mathrm{~Hz}, 1 \mathrm{H}\right), 0.99(\mathrm{~s}$, 12H), $2.31(\mathrm{~d}, J=11.5 \mathrm{~Hz}, 2 \mathrm{H}), 7.19-7.42(\mathrm{~m}, 10 \mathrm{H}) ;{ }^{13} \mathrm{C} \mathrm{NMR}\left(75 \mathrm{MHz}, \mathrm{CDCl}_{3}, \mathrm{rt}\right) \delta 4.6,10.0,24.3,38.2$, $83.2,125.8,126.8,127.7,128.0,128.51,128.54,141.2,144.2,151.8$. The carbon signal attached to B was not observed due to their low intensity; ${ }^{11} \mathrm{~B} \mathrm{NMR}\left(\mathrm{CDCl}_{3}, 96 \mathrm{MHz}, \mathrm{rt}\right) \delta 30.01$. MS (EI, m/z (relative intensity)): $360\left(\mathrm{M}^{+}, 43\right), 260$ (44), 232 (94), 143 (100), 101 (61). Anal. Calcd for $\mathrm{C}_{24} \mathrm{H}_{29} \mathrm{BO}_{2}: \mathrm{C}, 80.01 ; \mathrm{H}$, 8.11\%. Found: C, 79.59; H, 8.17\%. 


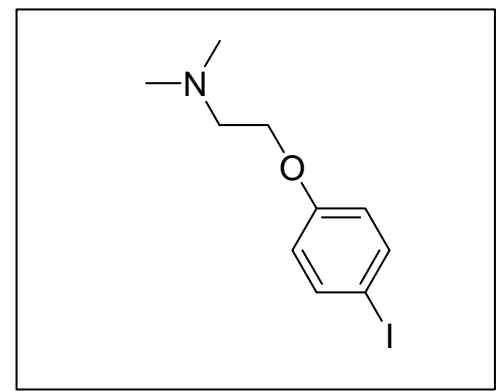

Preparation of $\boldsymbol{N}$-[2-(4-Iodophenoxy)ethyl]- $\boldsymbol{N}, \boldsymbol{N}$-dimethylamine. ${ }^{6}$ Potassium hydroxide $(226 \mathrm{mg}, 4$ mmol) was added to a solution of 4-iodophenol $(888 \mathrm{mg}, 4 \mathrm{mmol})$ in ethanol $(10 \mathrm{~mL})$ at room temperature. The reaction was stirred for $3 \mathrm{~h}$ and the solvent evaporated to afford 4-iodophenoxide salt as a yellow oil. Sodium hydroxide (200 mg, $5 \mathrm{mmol})$ in water $(1 \mathrm{~mL})$ was added to a cold solution of 2dimethylaminoethylchloride hydrochloride $(600 \mathrm{mg}, 4.2 \mathrm{mmol})$ in water $(2 \mathrm{~mL})$ at $0{ }^{\circ} \mathrm{C}$. The reaction mixture was saturated with solid $\mathrm{NaCl}$ and extracted with toluene $(5 \times 3 \mathrm{~mL})$. The combined toluene extracts were dried over $\mathrm{KOH}$ and filtered. The resulting solution was added to the 4-iodophenoxide salt and refluxed for 12 h. The reaction was cooled to room temperature and filtered. The filtrate was washed with saturated aqueous $\mathrm{NaOH}(3 \times 10 \mathrm{~mL})$ and brine $(5 \mathrm{~mL})$. The organic phase was dried, filtered, and purified by bulb to bulb distillation (160-170 ${ }^{\circ} \mathrm{C} / 2.8$ Torr) to give $703 \mathrm{mg}(2.4 \mathrm{mmol}, 60 \%)$ of the title compound as a brown oil. ${ }^{1} \mathrm{H}$ NMR $\left(\mathrm{CDCl}_{3}, 300 \mathrm{MHz}, \mathrm{rt}\right) \delta 2.33(\mathrm{~s}, 6 \mathrm{H}), 2.72(\mathrm{t}, J=5.7 \mathrm{~Hz}, 2 \mathrm{H}), 4.02(\mathrm{~d}, J=5.7 \mathrm{~Hz}, 2 \mathrm{H}), 6.66-6.70(\mathrm{~m}$, 2H), 7.52-7.56 (m, 2H). ${ }^{1} \mathrm{H}$ NMR spectrum in agreement with literature.

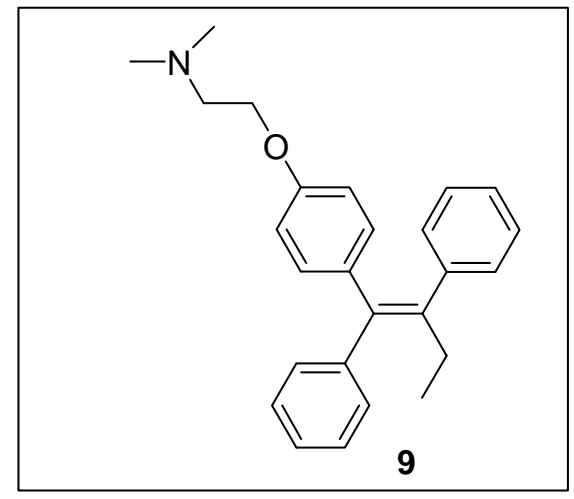

(Z)-Tamoxifen ((Z)-2-[4-(1,2-Diphenyl-1-butenyl)phenoxy]-N,N-dimethylethanamine) (9). [10540-29-

(6) Tessier, P. E.; Penwell, A. J.; Souza, F. E. S.; Fallis, A. G. Org.Lett. 2003, 5, 2989-2992. 
1] $]^{7}$ A mixture of $5 a(18 \mathrm{mg}, 0.054 \mathrm{mmol}), N$-[2-(4-iodophenoxy)ethyl]- $N, N$-dimethylamine (70 mg, 0.24 $\mathrm{mmol}$ ), $\mathrm{Pd}(\mathrm{dba})_{2}(12 \mathrm{mg}, 0.02 \mathrm{mmol}), 0.1 \mathrm{M} \mathrm{P}^{t} \mathrm{Bu}_{3} /$ hexane $(400 \mu \mathrm{l}, 0.04 \mathrm{mmol})$, and $3 \mathrm{M} \mathrm{NaOH}$ aqueous solution $(300 \mu \mathrm{L}, 0.9 \mathrm{mmol})$ in THF $(2 \mathrm{~mL})$ was stirred at $60{ }^{\circ} \mathrm{C}$ for $48 \mathrm{~h}$. The reaction mixture was quenched with saturated aq. $\mathrm{NH}_{4} \mathrm{Cl}(1 \mathrm{~mL})$. The resulting mixture was diluted with chloroform $(10 \mathrm{~mL})$ and washed with water $(3 \mathrm{~mL})$. The organic layer was separated, dried over anhydrous magnesium sulfate, and concentrated under vacuum. The crude product was purified by column chromatography $\left(\mathrm{CHCl}_{3} / \mathrm{MeOH} / \mathrm{Et}_{3} \mathrm{~N}=100: 10: 1\right)$ to give 9 as pale yellow solid (52 mg, $0.14 \mathrm{mmol}, 70 \%$ yield). FT-IR $\left(\mathrm{KBr}, \mathrm{cm}^{-1}\right): 3021(\mathrm{~m}), 2930(\mathrm{~m}), 2761$ (m), $1610(\mathrm{w}), 1512$ (m), 1247 (m). ${ }^{1} \mathrm{H}$ NMR (300 MHz, CDCl 3 , rt) $\delta 0.93$ (t, $\left.J=7.5 \mathrm{~Hz}, 3 \mathrm{H}\right), 2.33(\mathrm{~s}, 6 \mathrm{H})$, $2.46(\mathrm{q}, J=7.5 \mathrm{~Hz}, 2 \mathrm{H}), 2.69$ (t, $J=5.7 \mathrm{~Hz}, 2 \mathrm{H}), 3.95(\mathrm{t}, J=5.7 \mathrm{~Hz}, 2 \mathrm{H}), 6.56(\mathrm{~d}, J=8.7 \mathrm{~Hz}, 2 \mathrm{H}), 6.77$ (d, $J=$ $8.7 \mathrm{~Hz}, 2 \mathrm{H}), 7.11-7.37(\mathrm{~m}, 10 \mathrm{H}) ;{ }^{13} \mathrm{C} \mathrm{NMR}\left(75 \mathrm{MHz}, \mathrm{CDCl}_{3}, \mathrm{rt}\right) \delta 13.6,29.0,45.7,58.2,65.4,113.3,126.0$, $126.5,127.8,128.1,129.4,129.7,131.8,135.6,138.1,141.3,142.4,143.8,156.6$. MS (EI, m/z (relative intensity)): $371\left(\mathrm{M}^{+}, 23\right), 252(5), 165(5), 152(4), 129(4), 72$ (100).

(7) Itami, K.; Kamei, T.; Yoshida, J.-i. J. Am. Chem. Soc. 2003, 125, 14670-14671. 

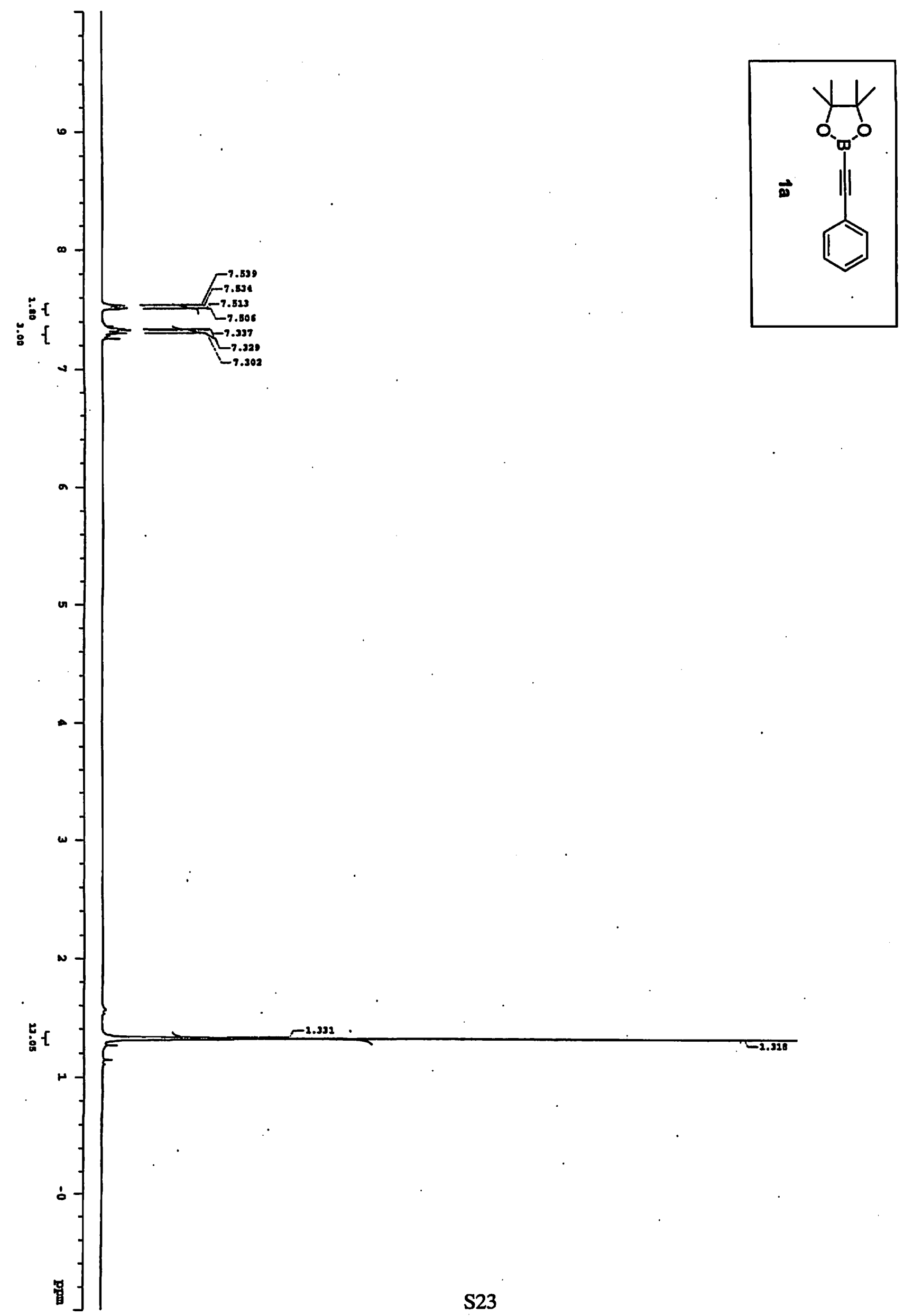


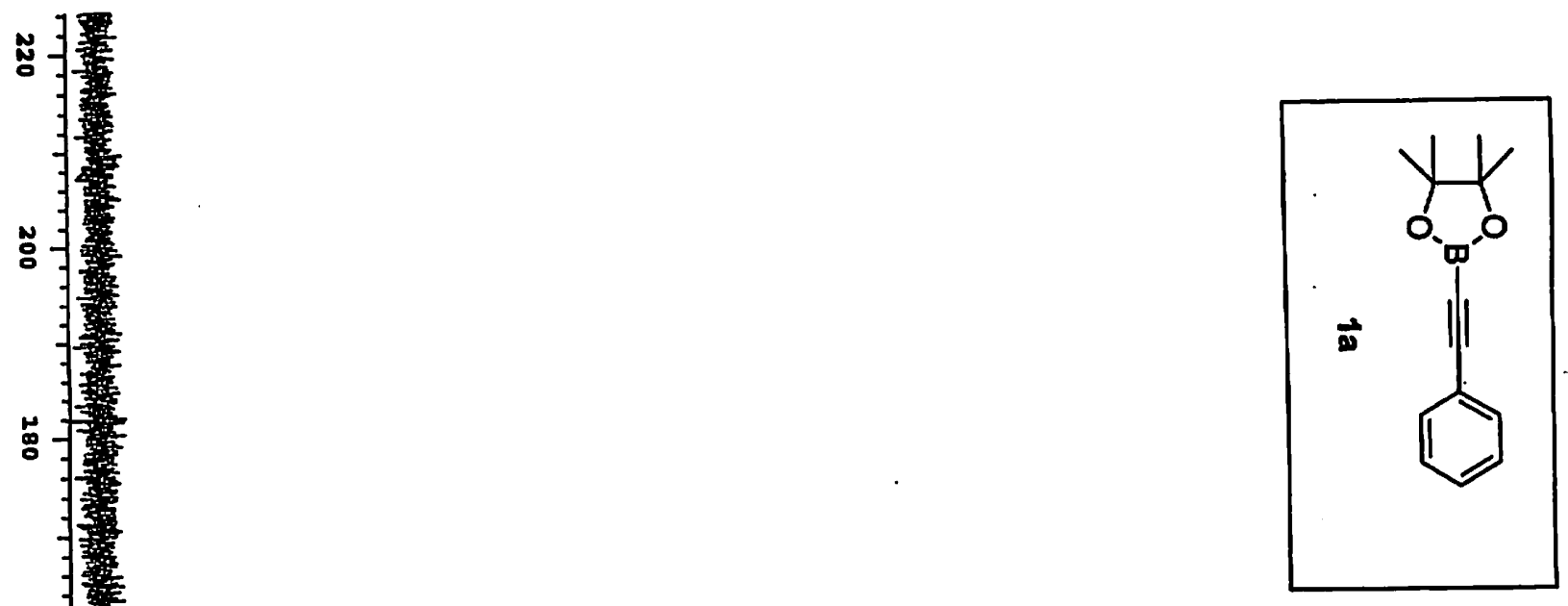

品手

呰

:

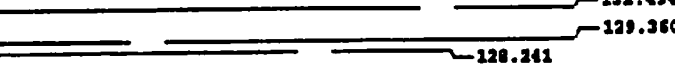

의

\&

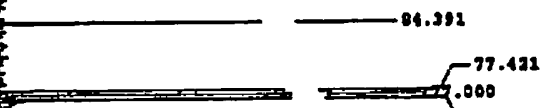

.000

a -

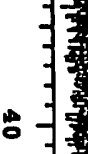

控

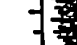

- 


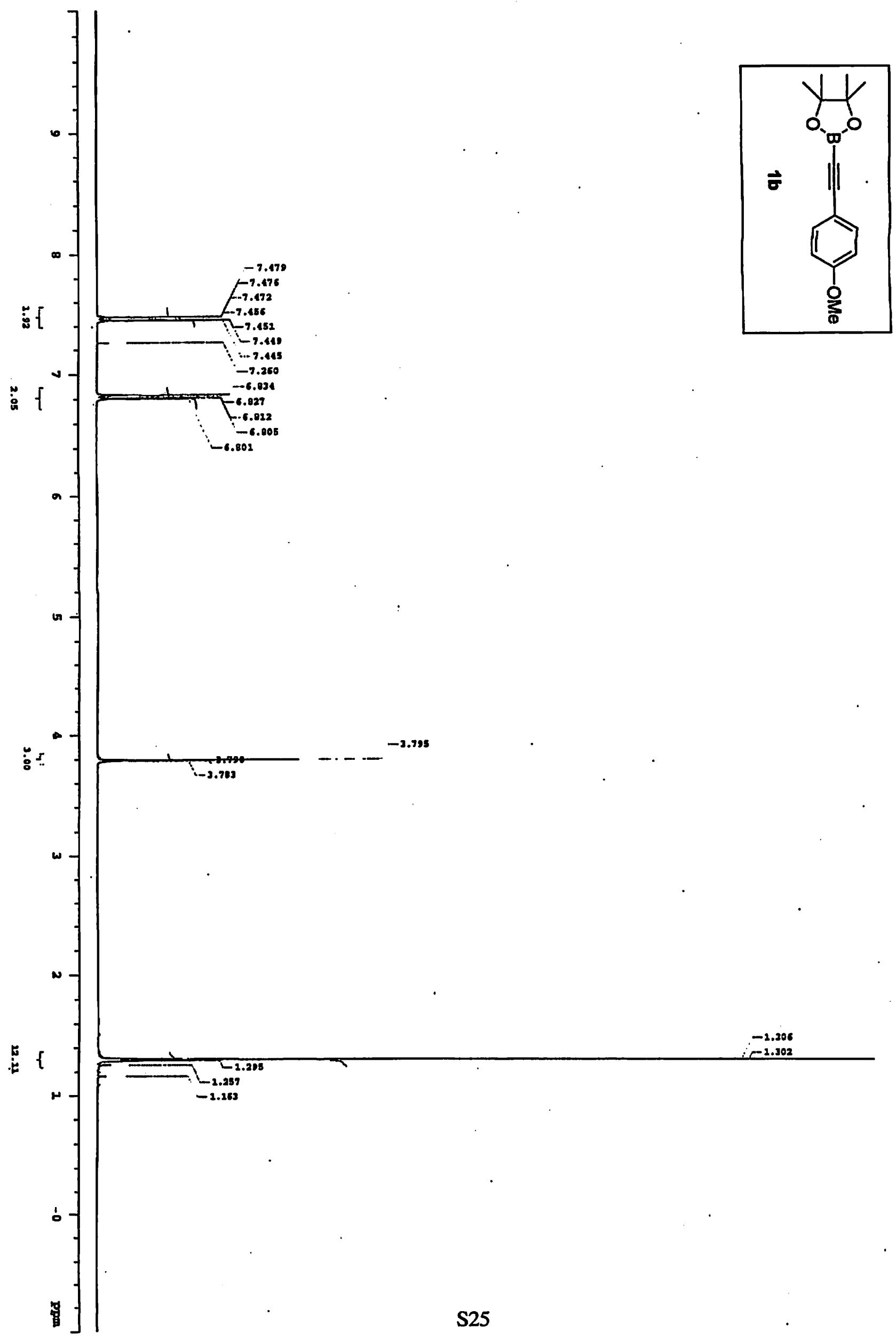




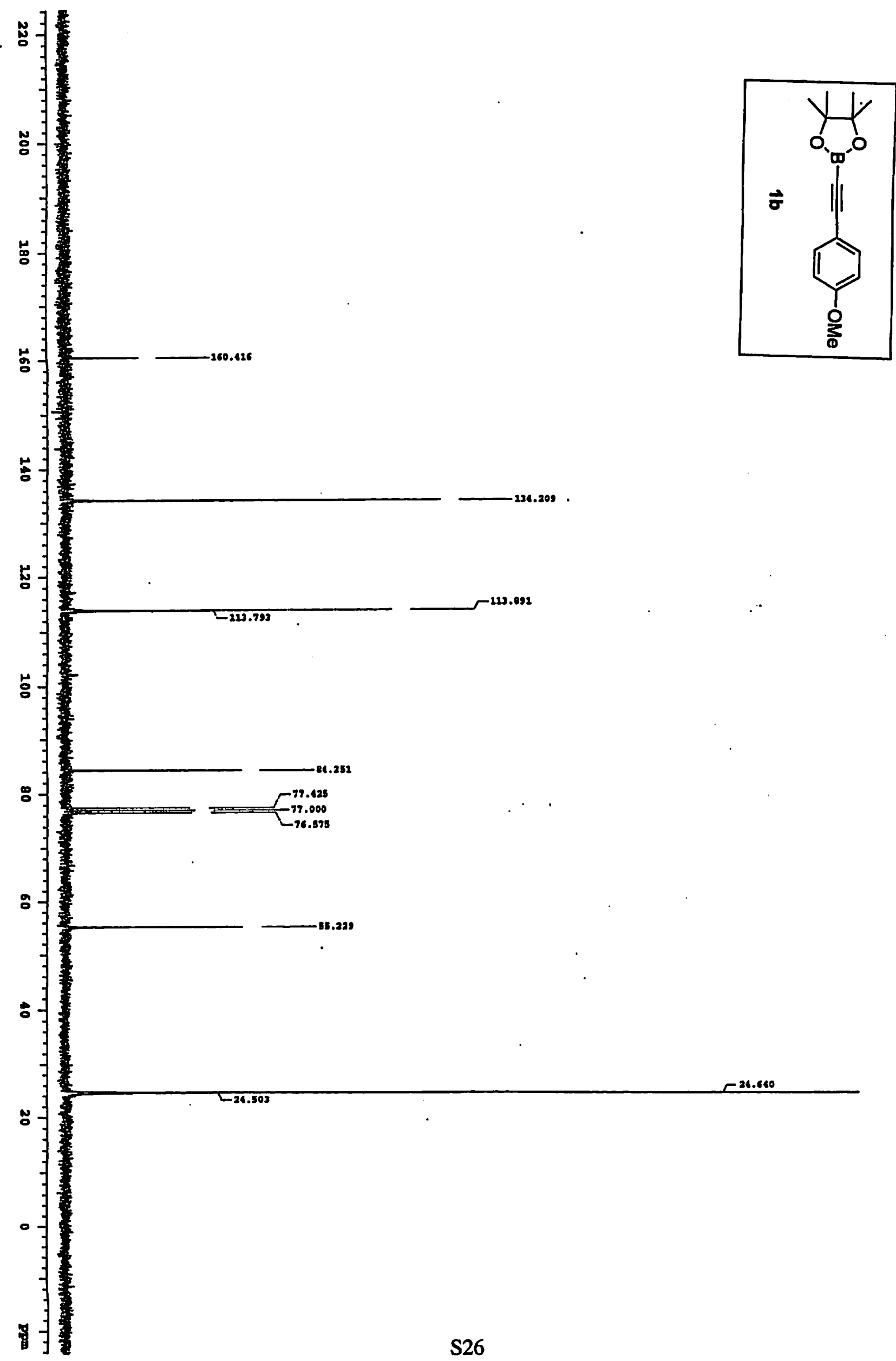




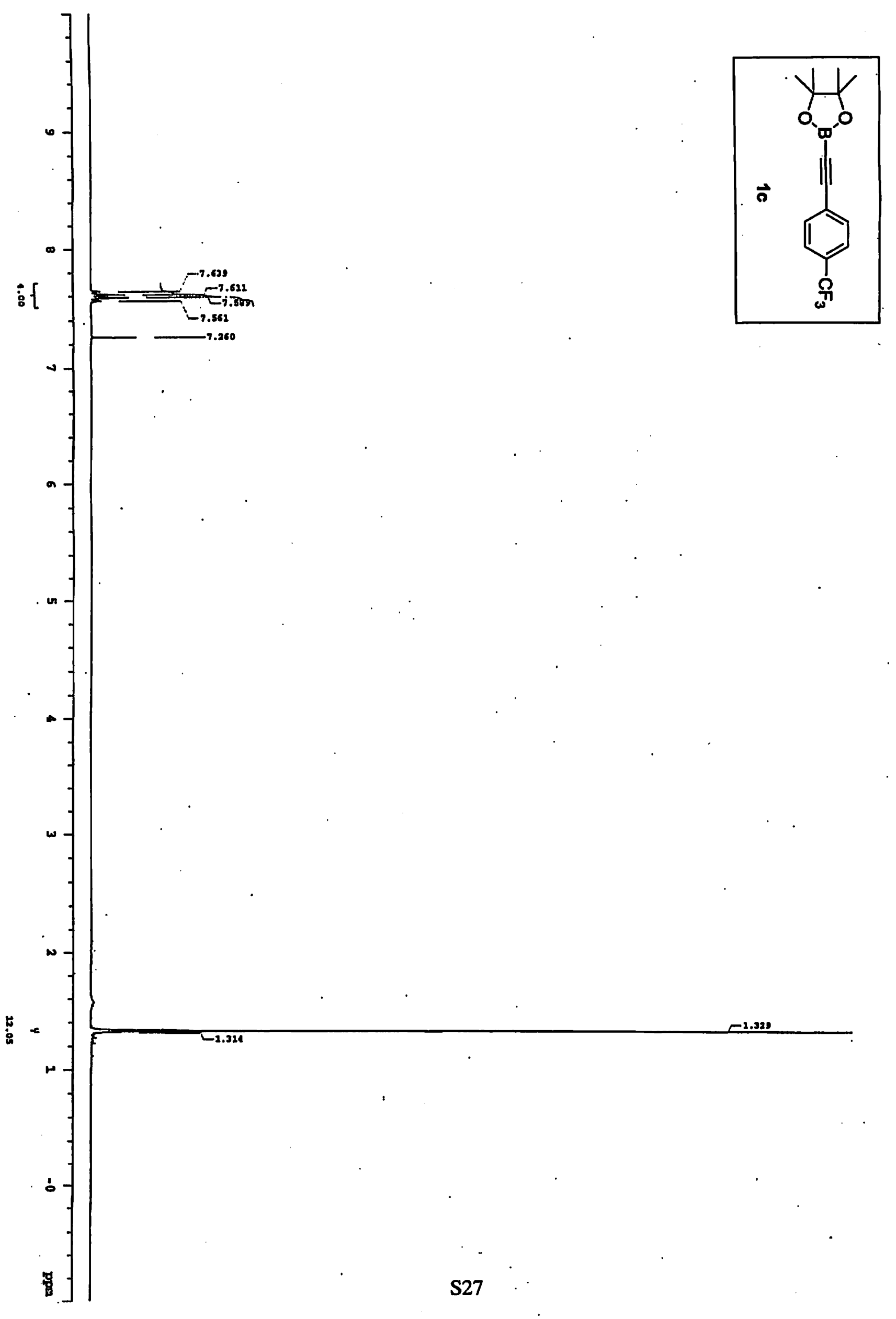




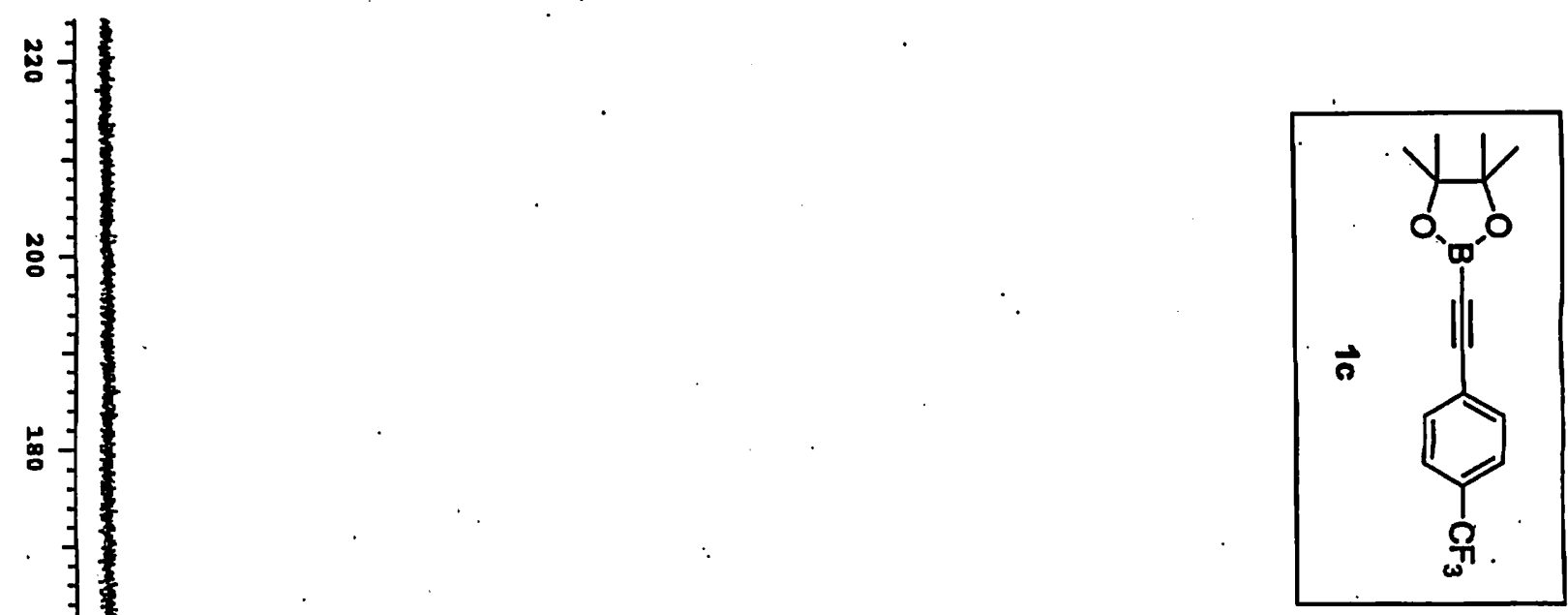

용

。․

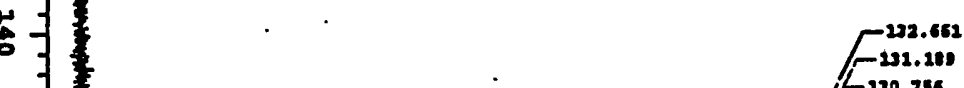

$F_{123.502}^{225.600}$

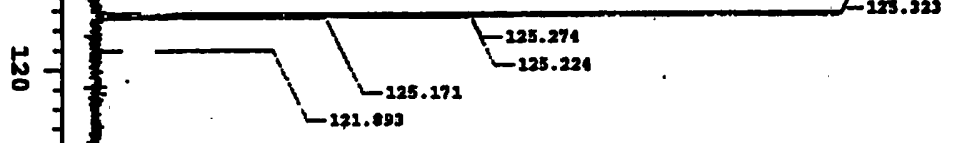

:

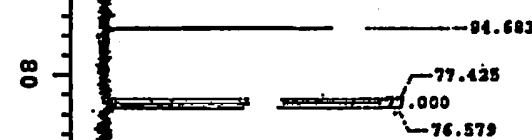

s -

ธ

:

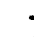

$:-$

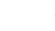

1

菌 


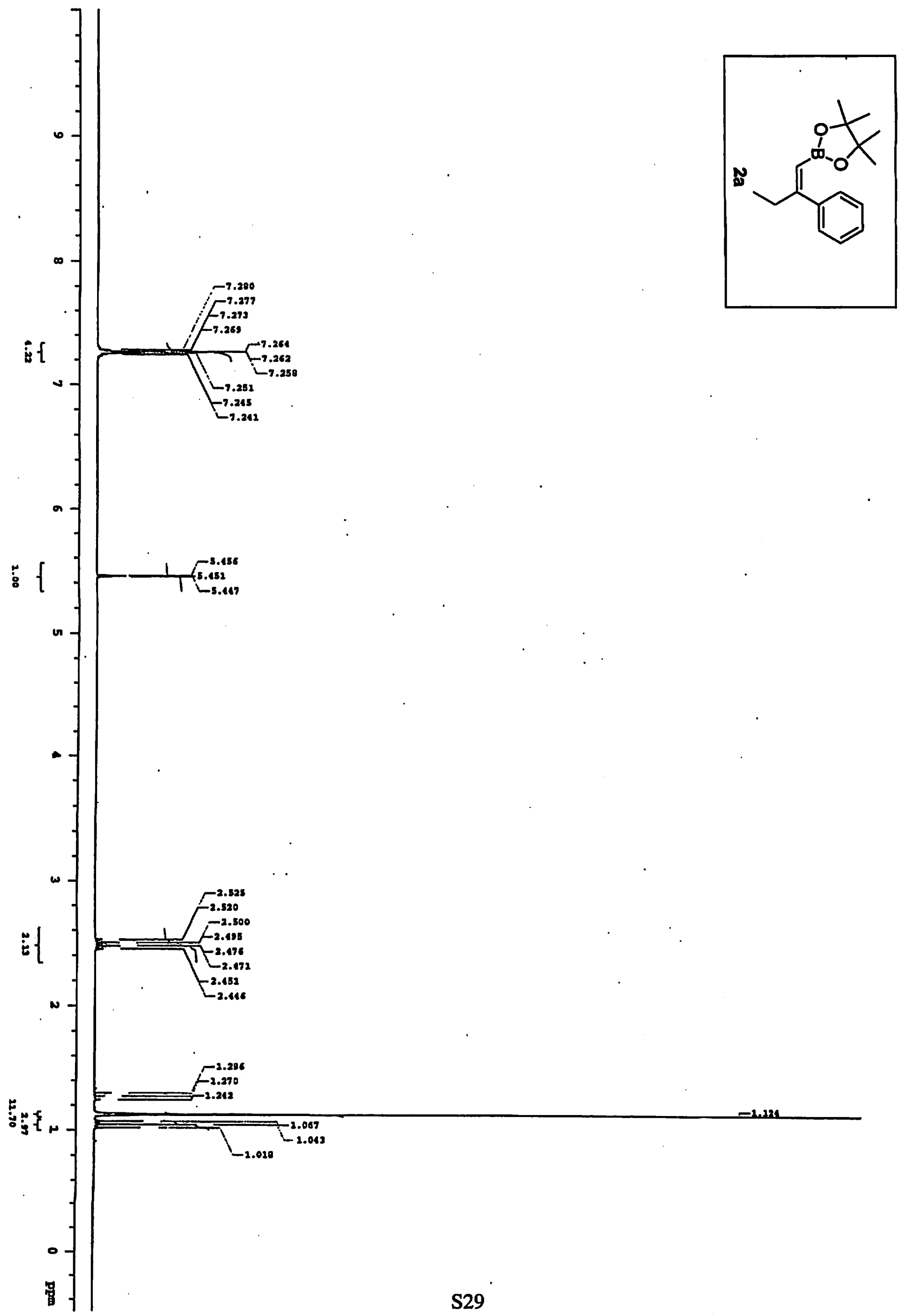




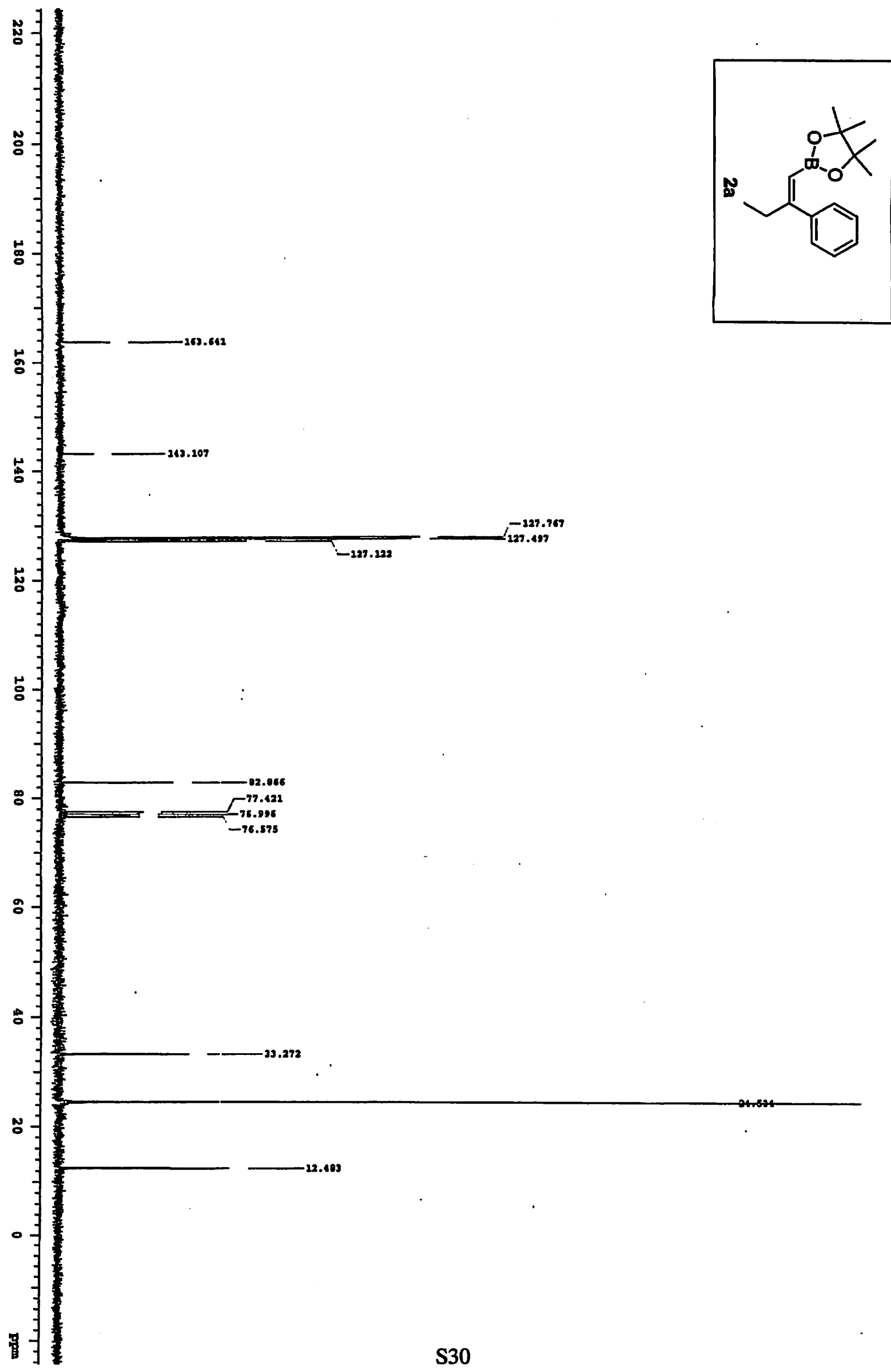




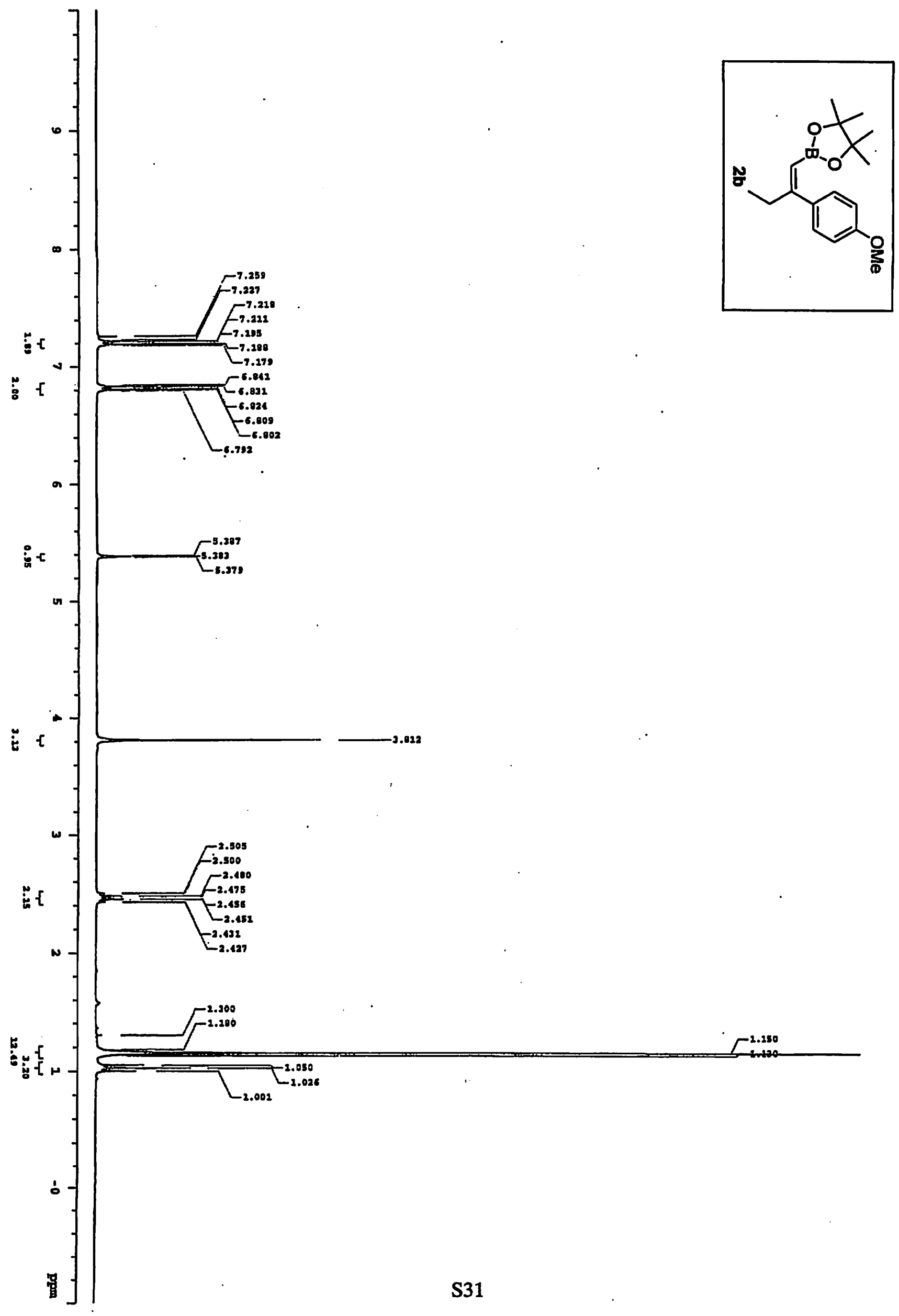




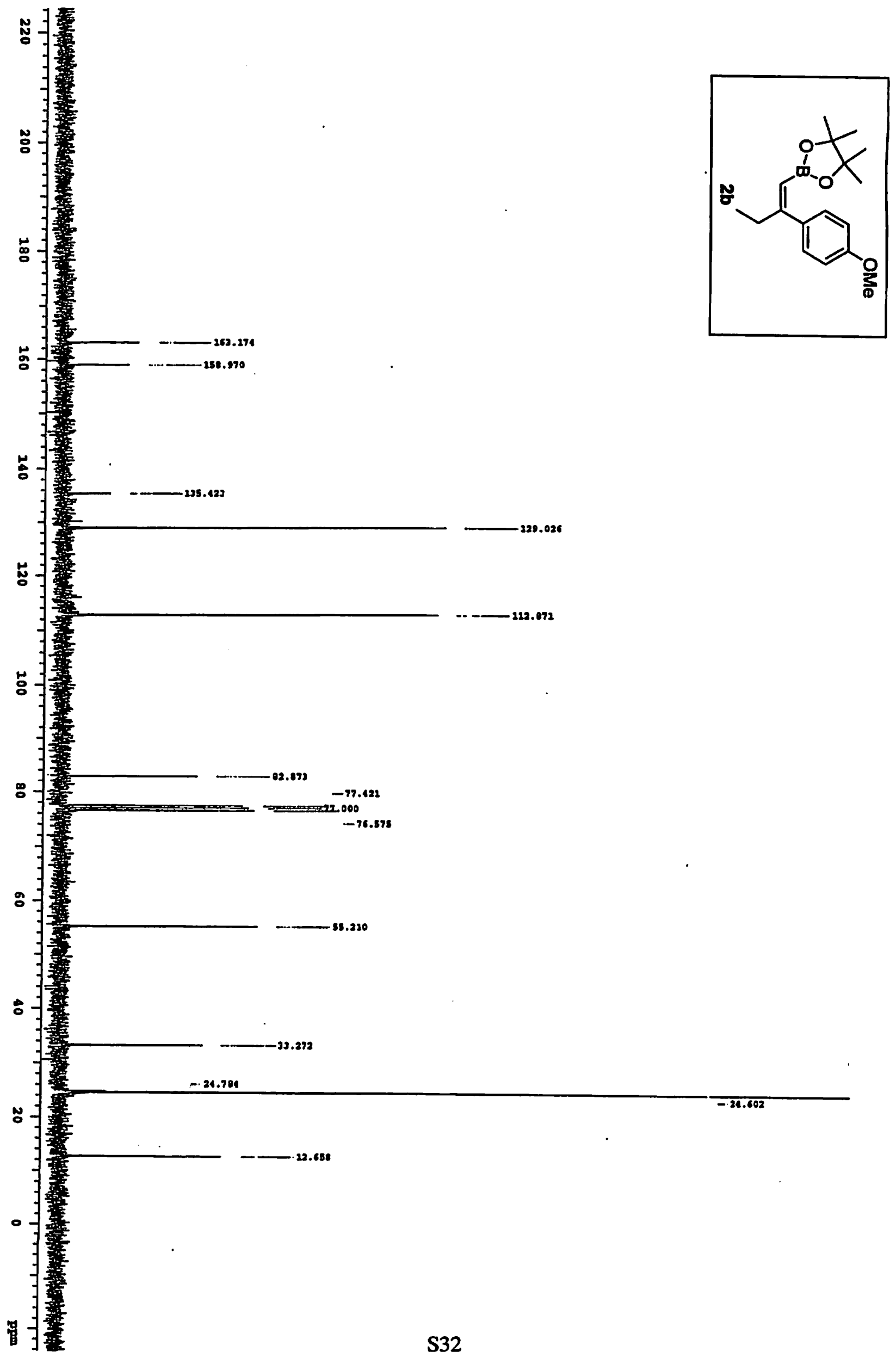




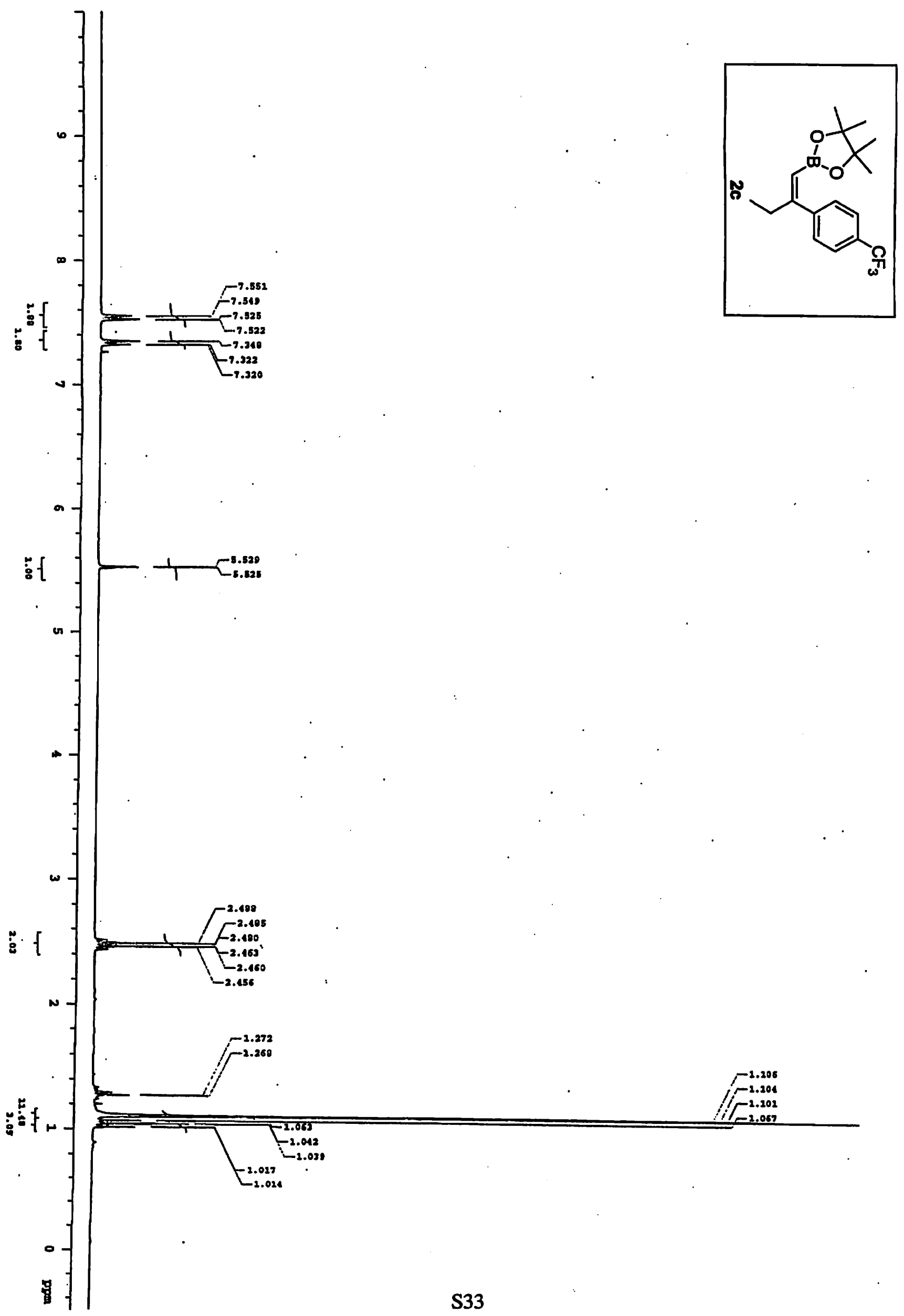




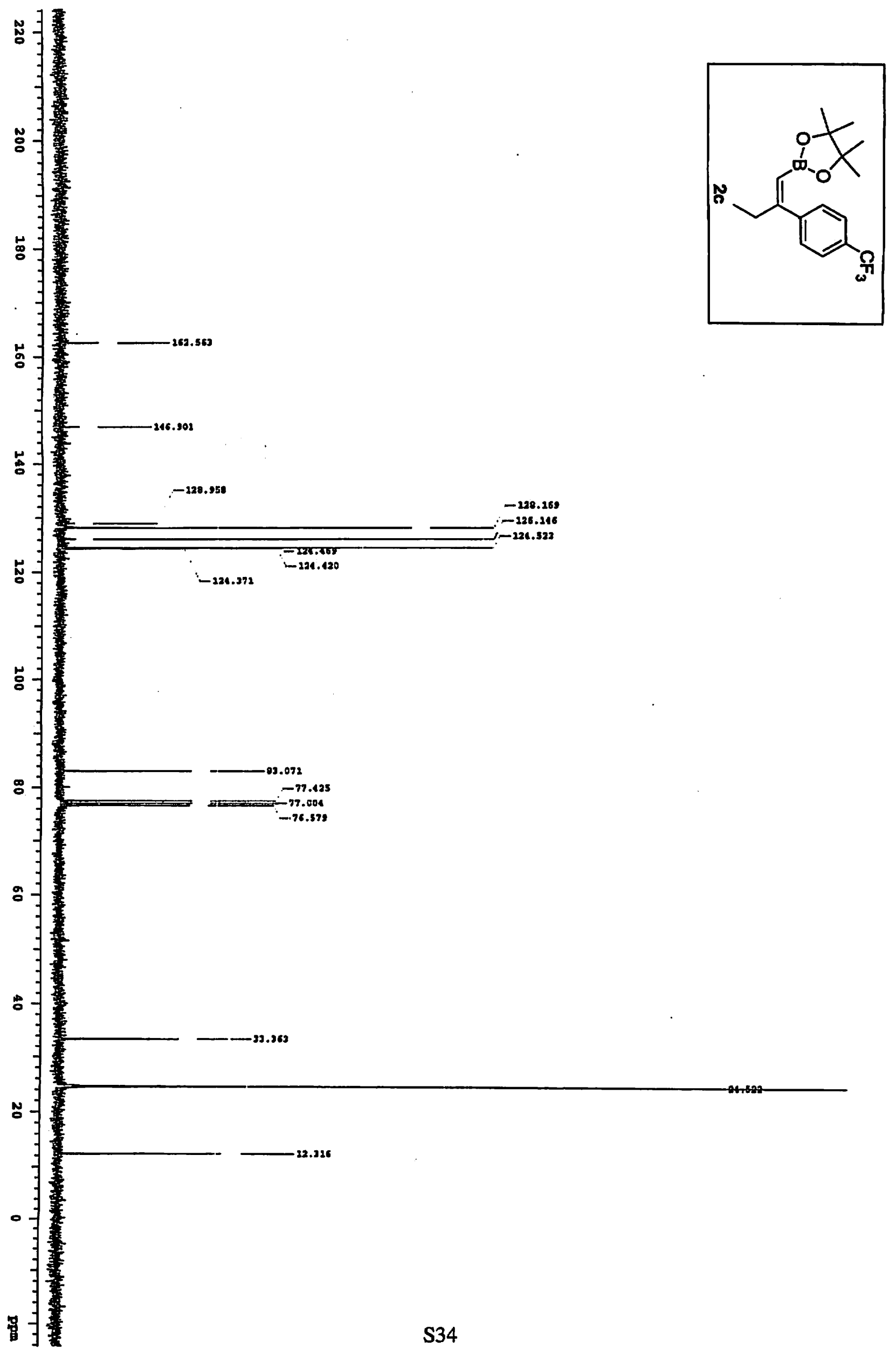




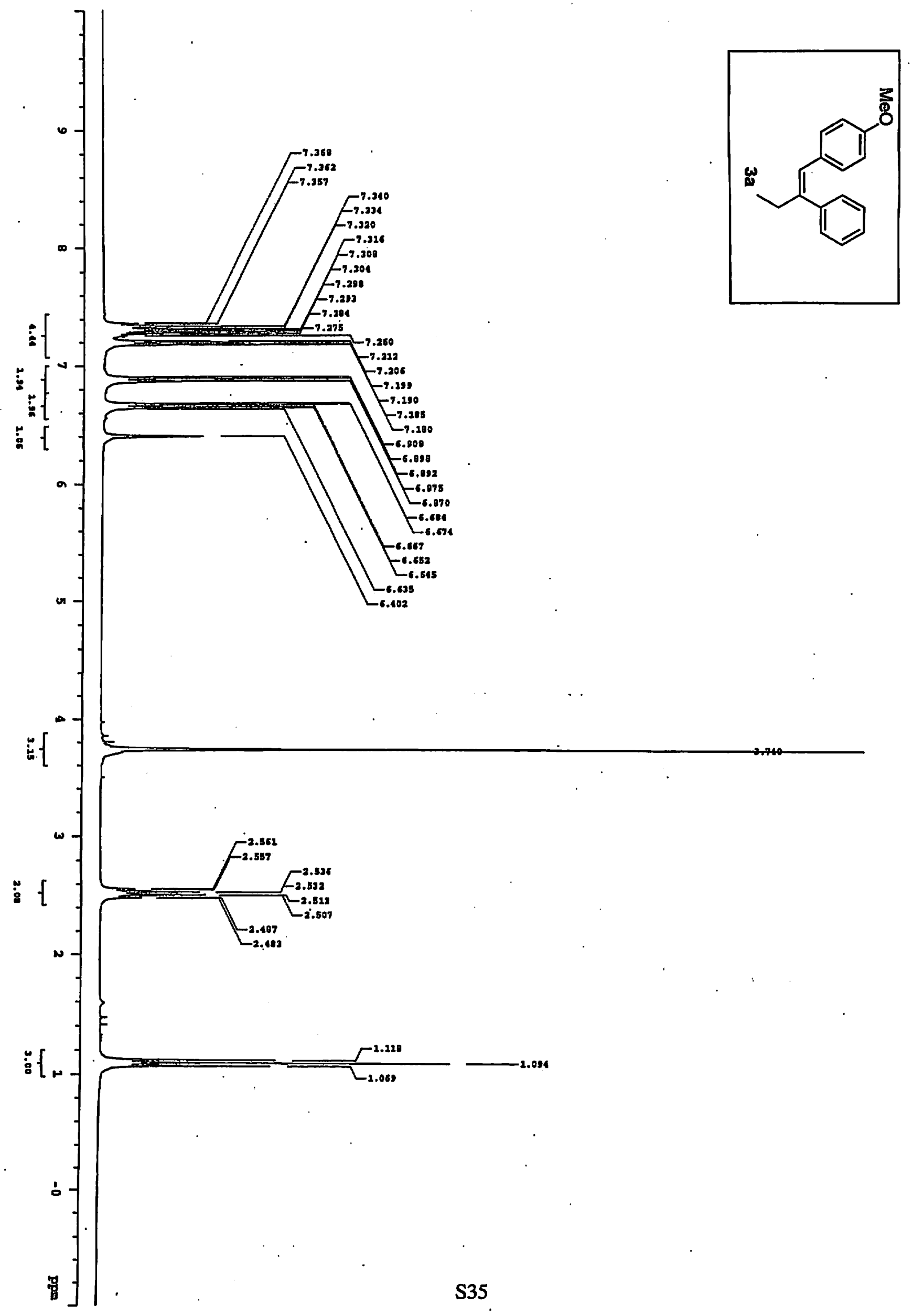




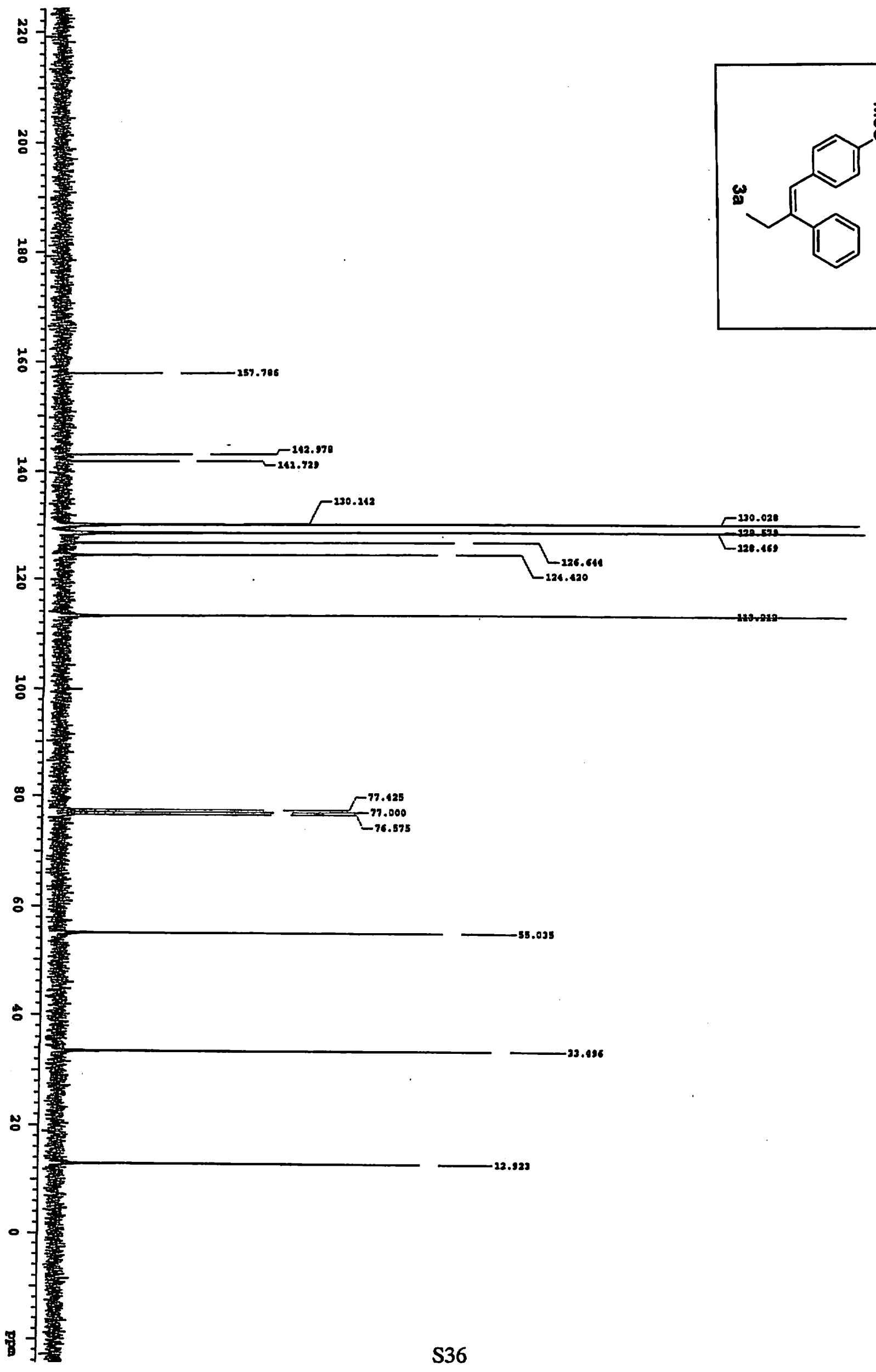




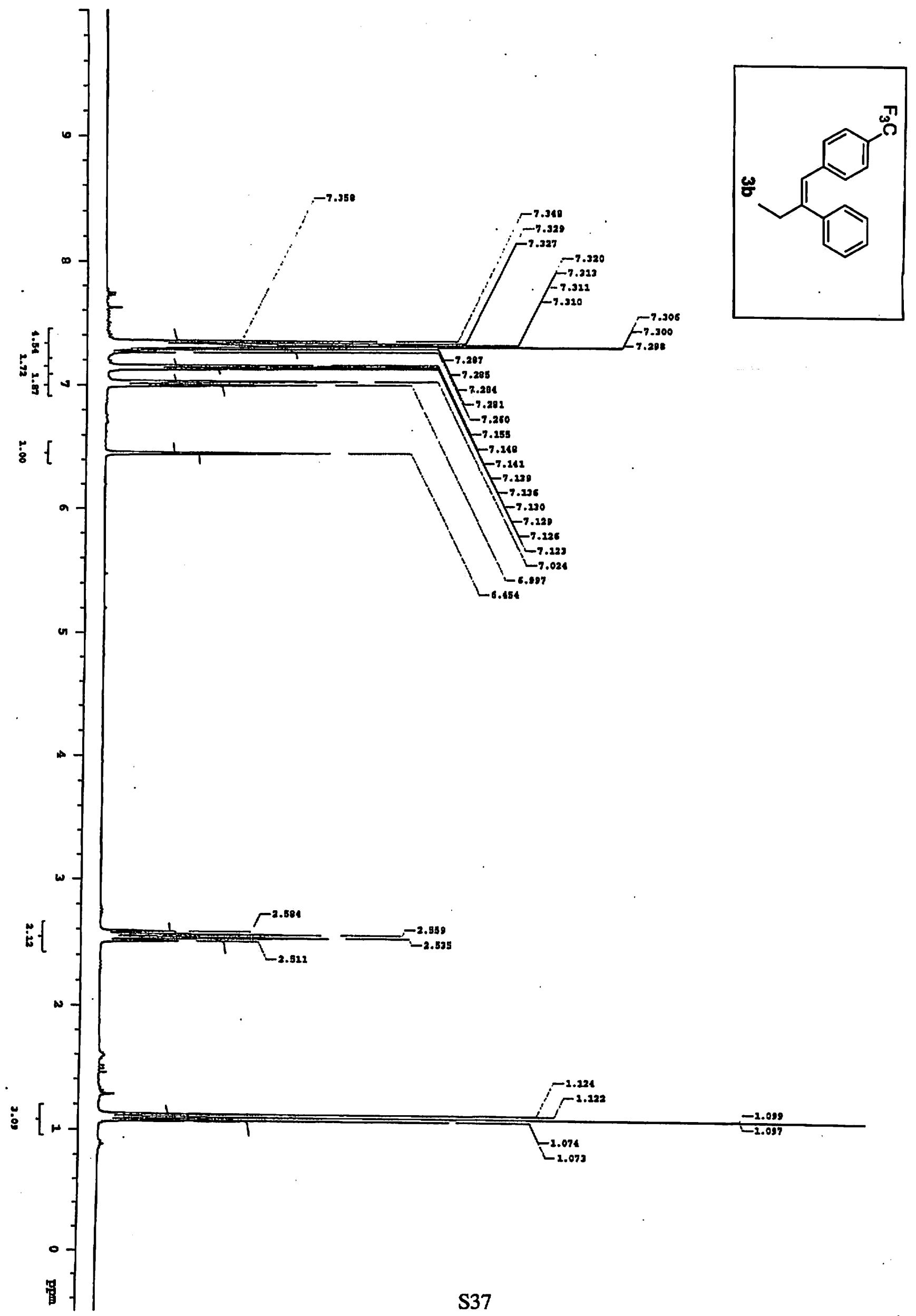




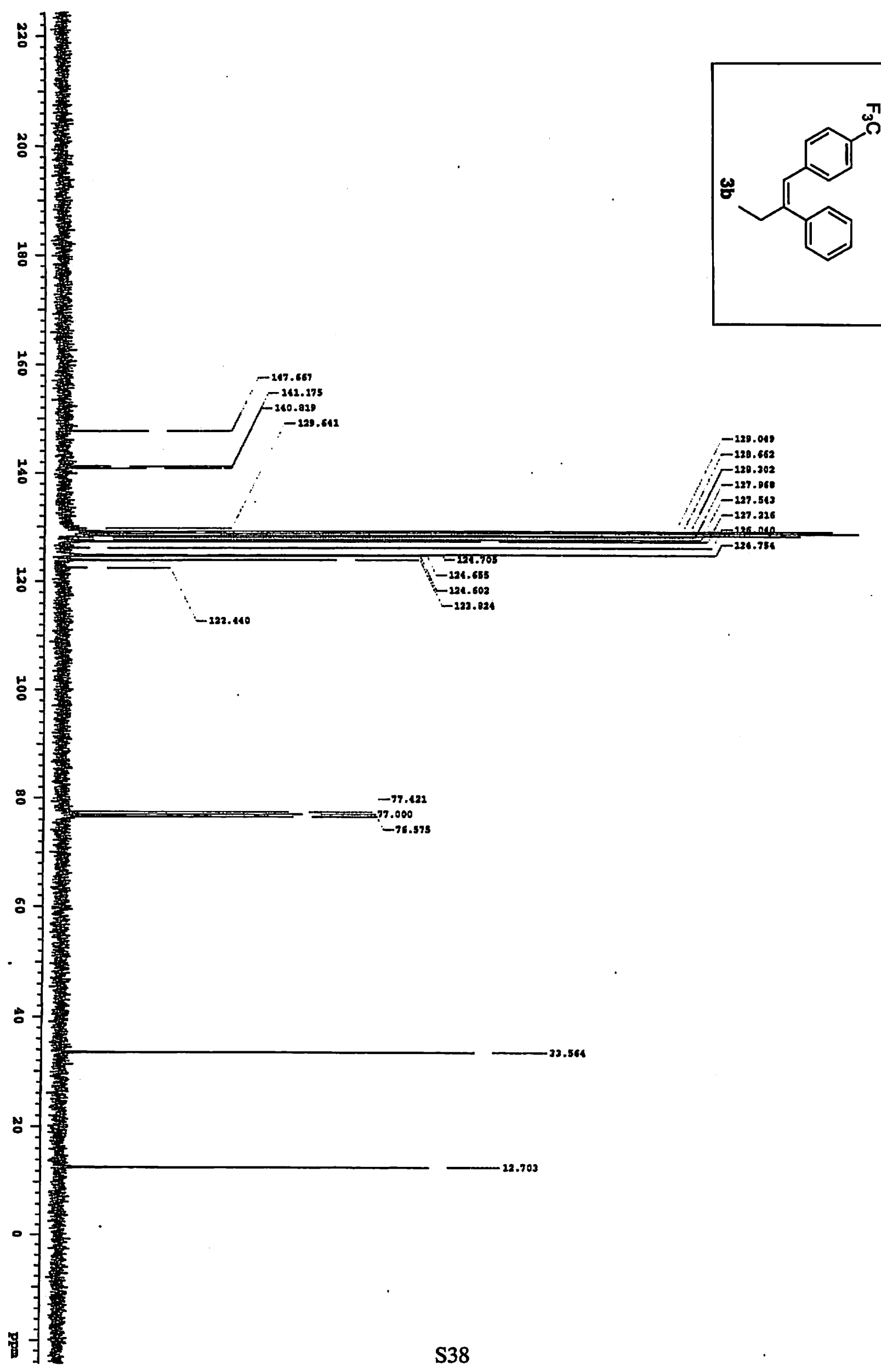



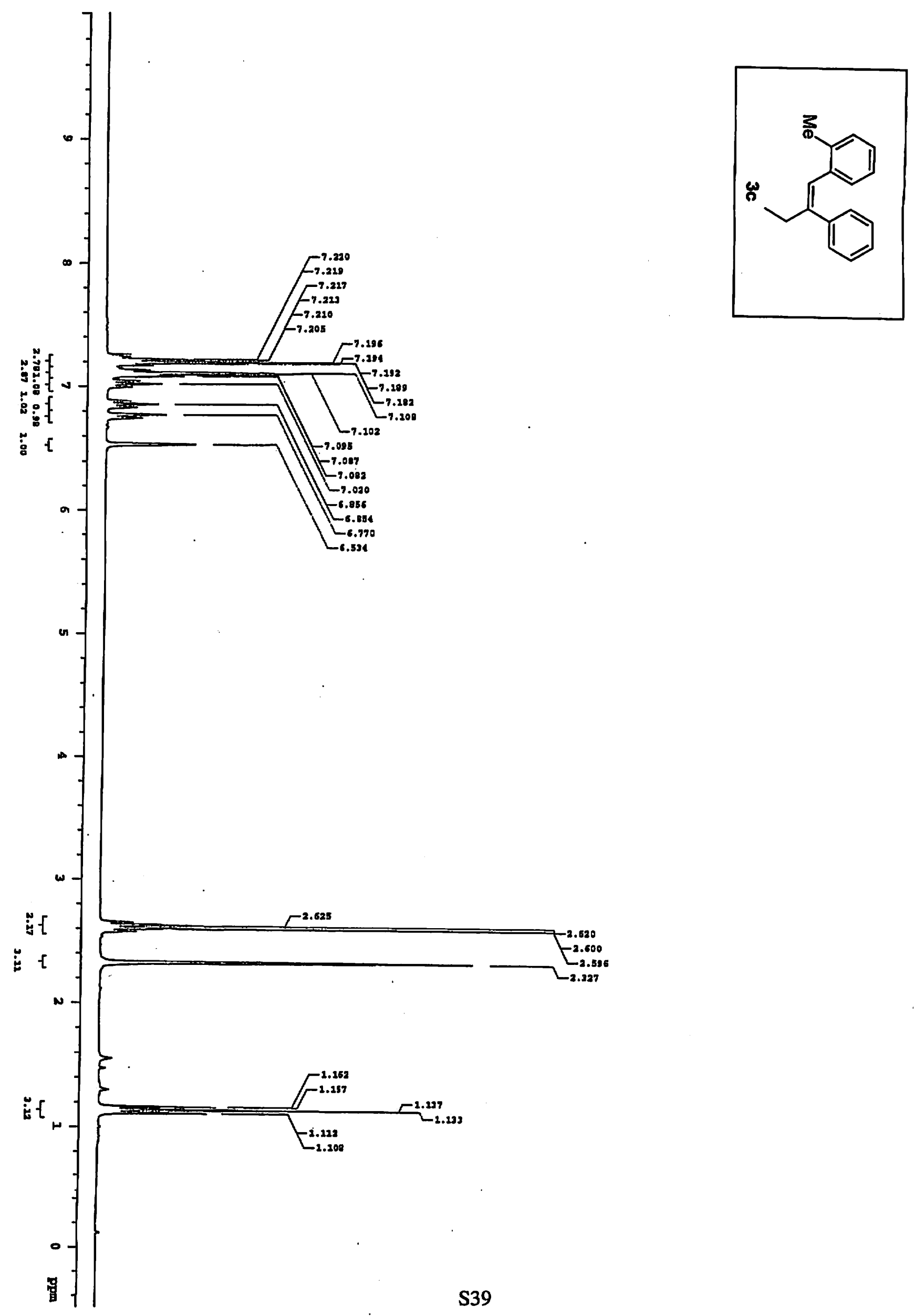


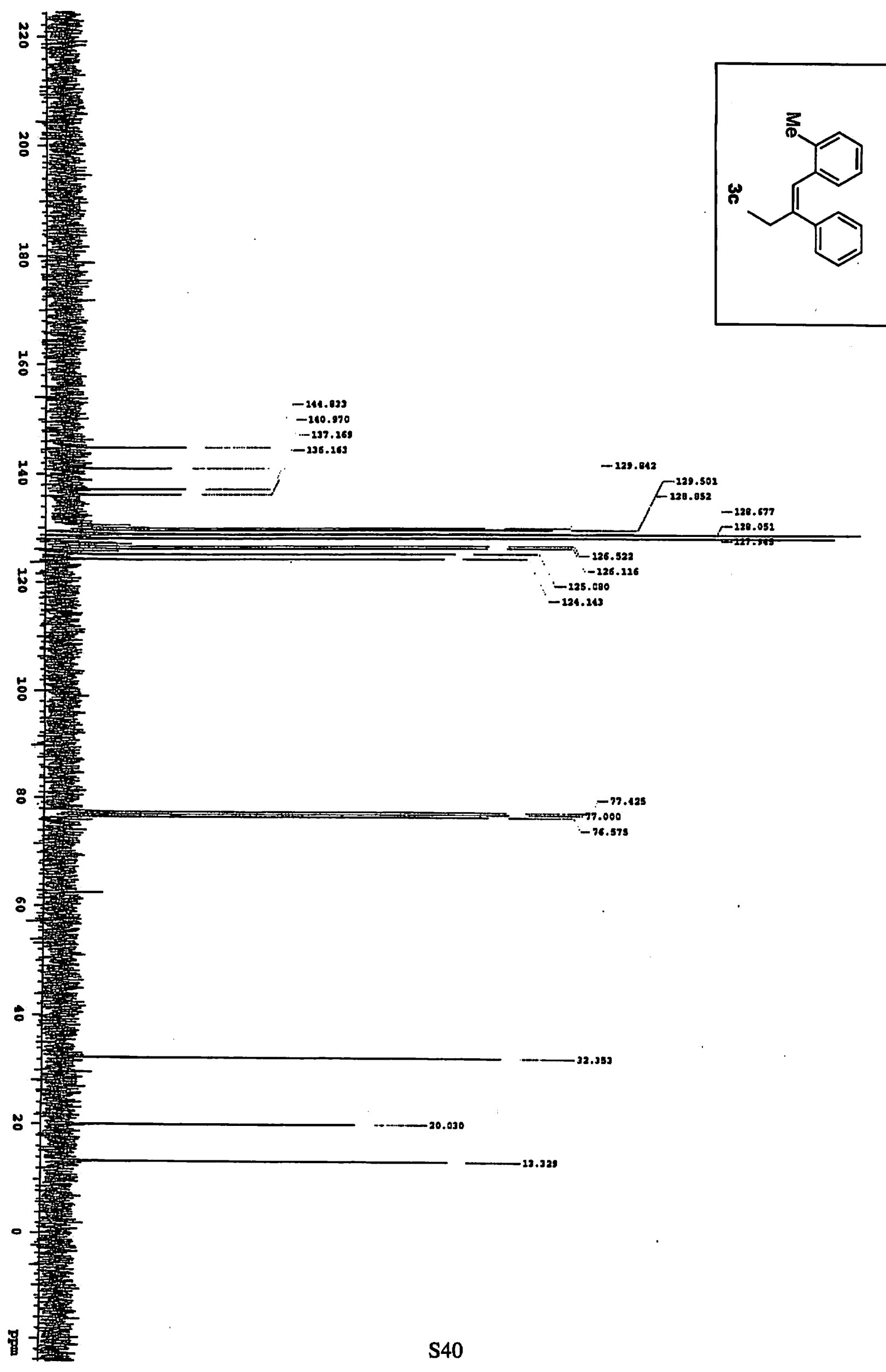



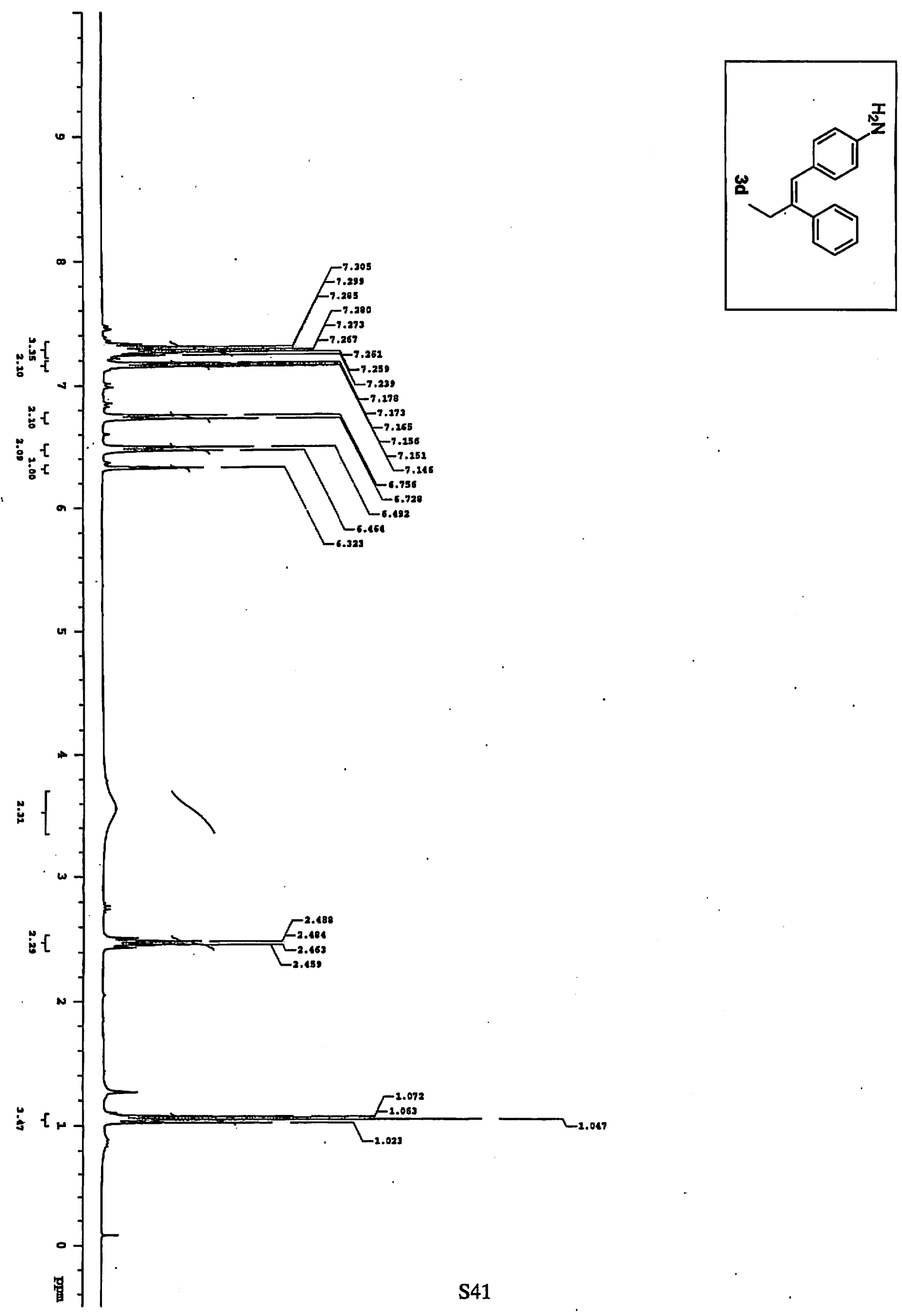


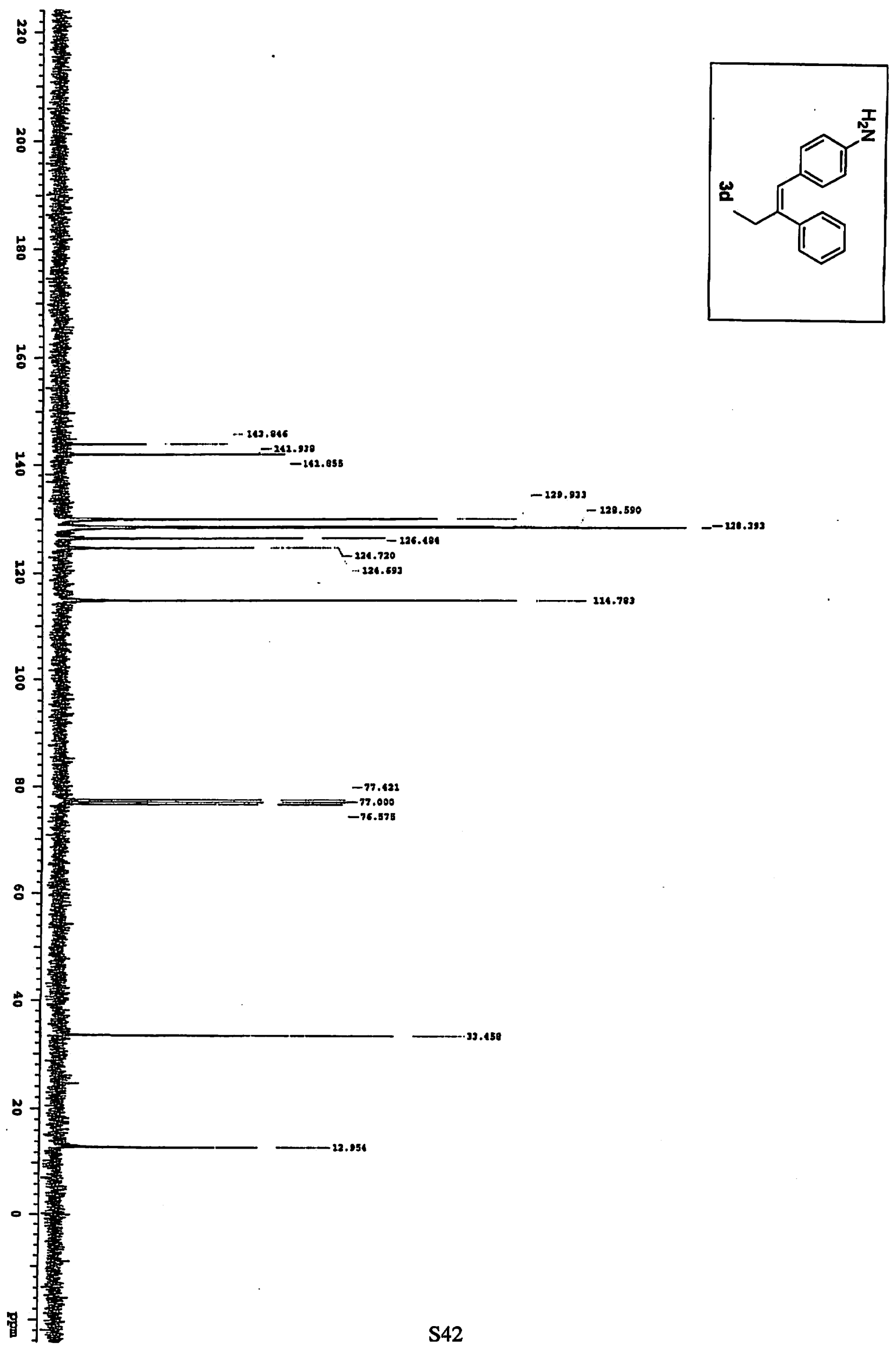




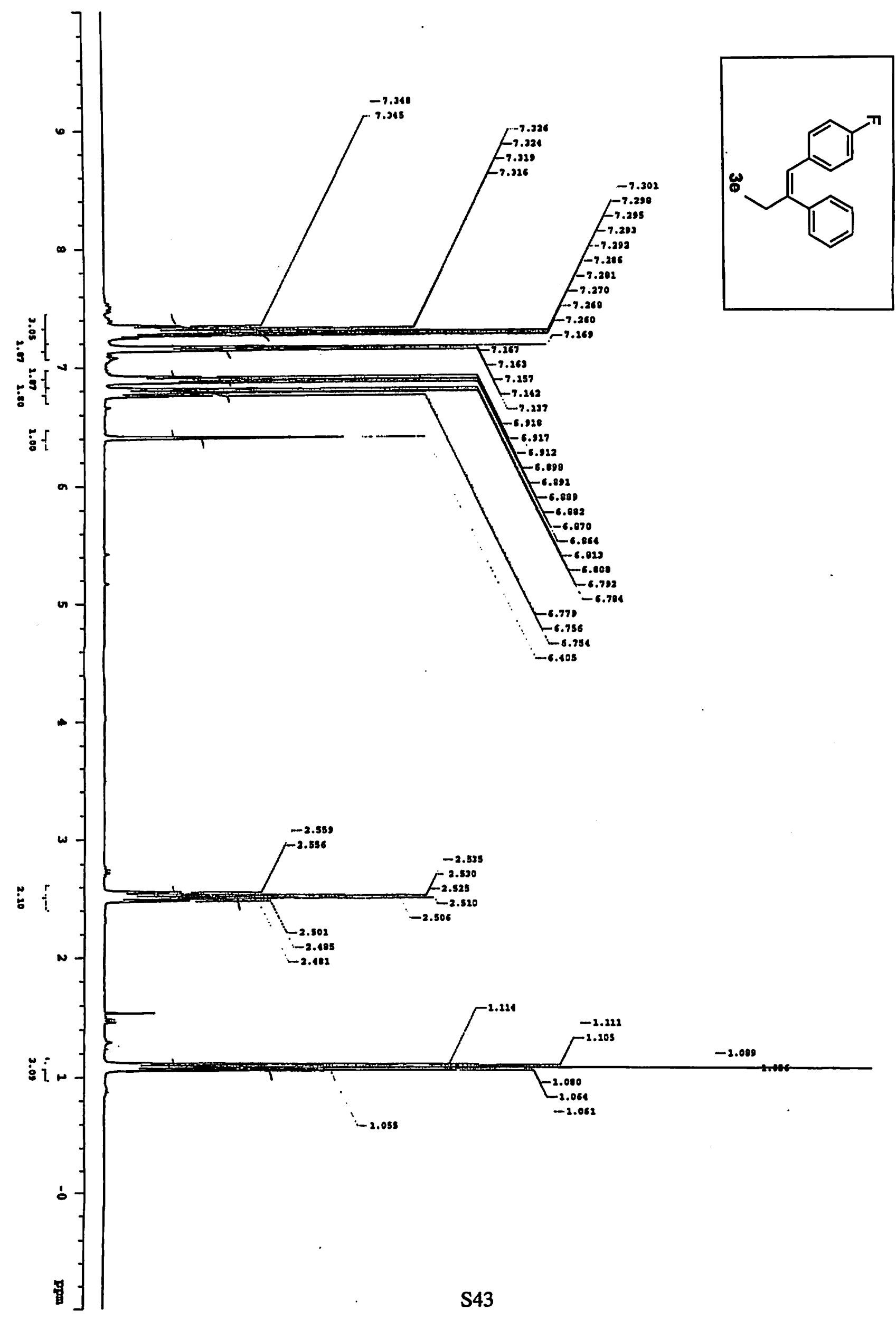




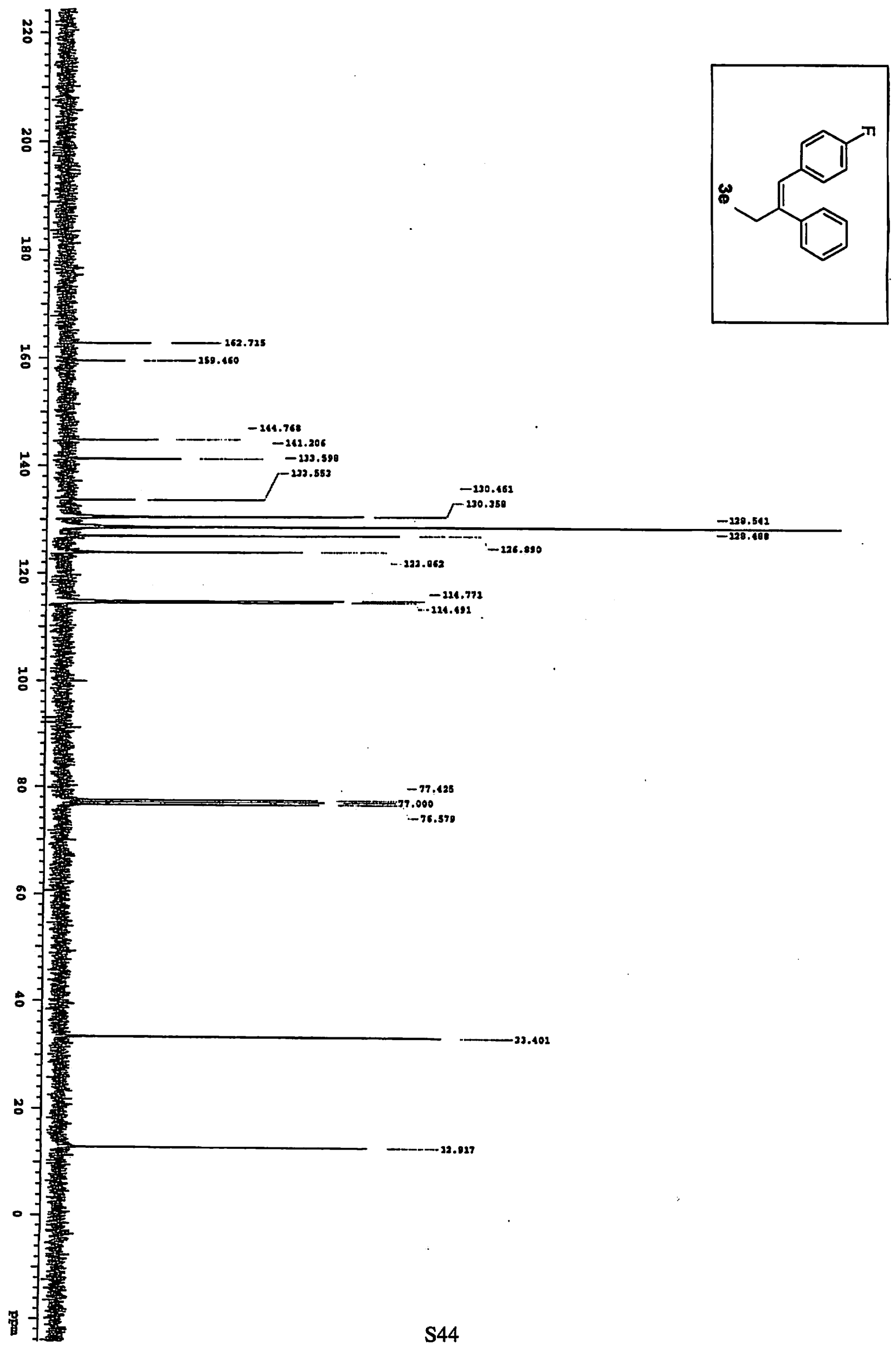




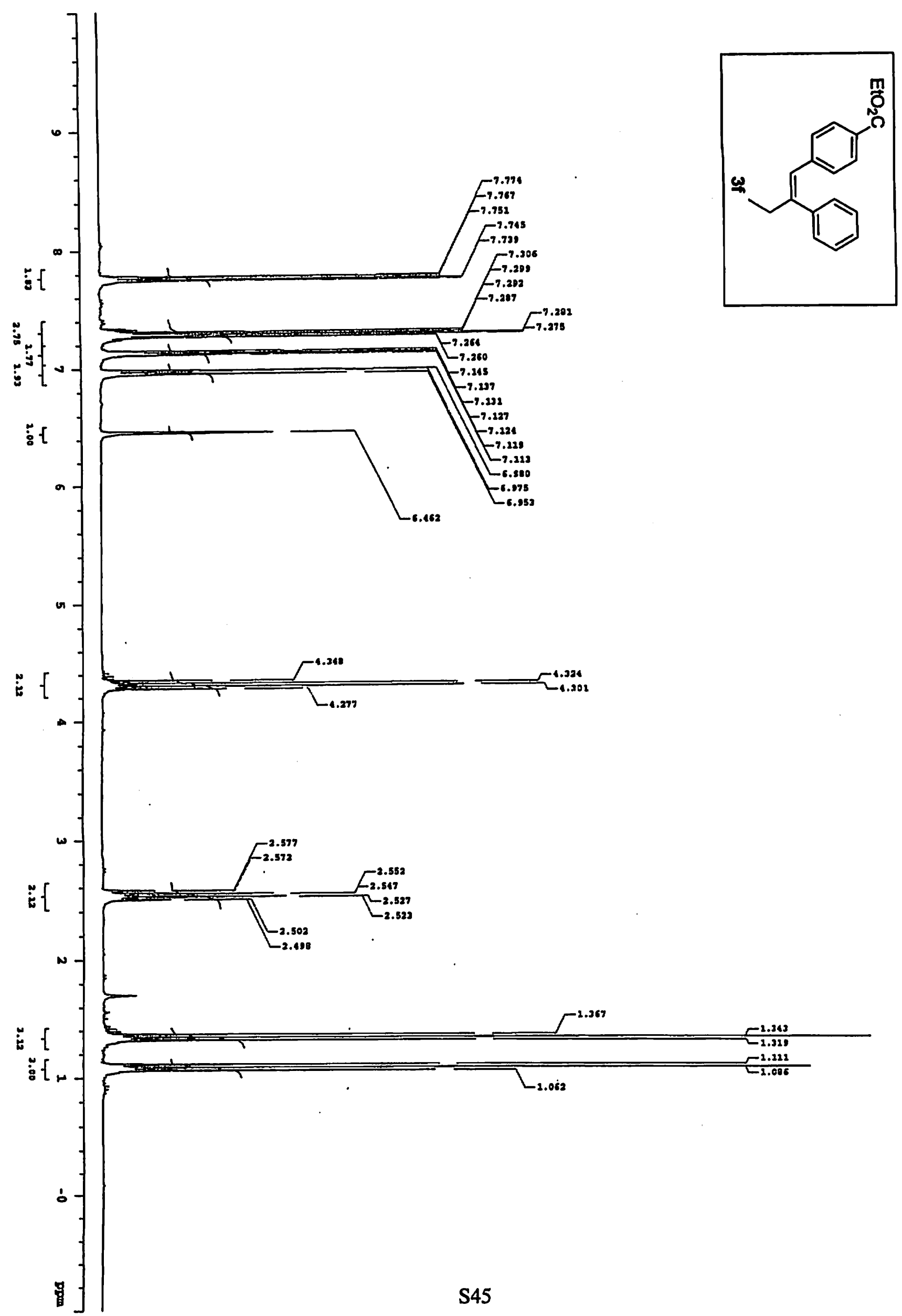




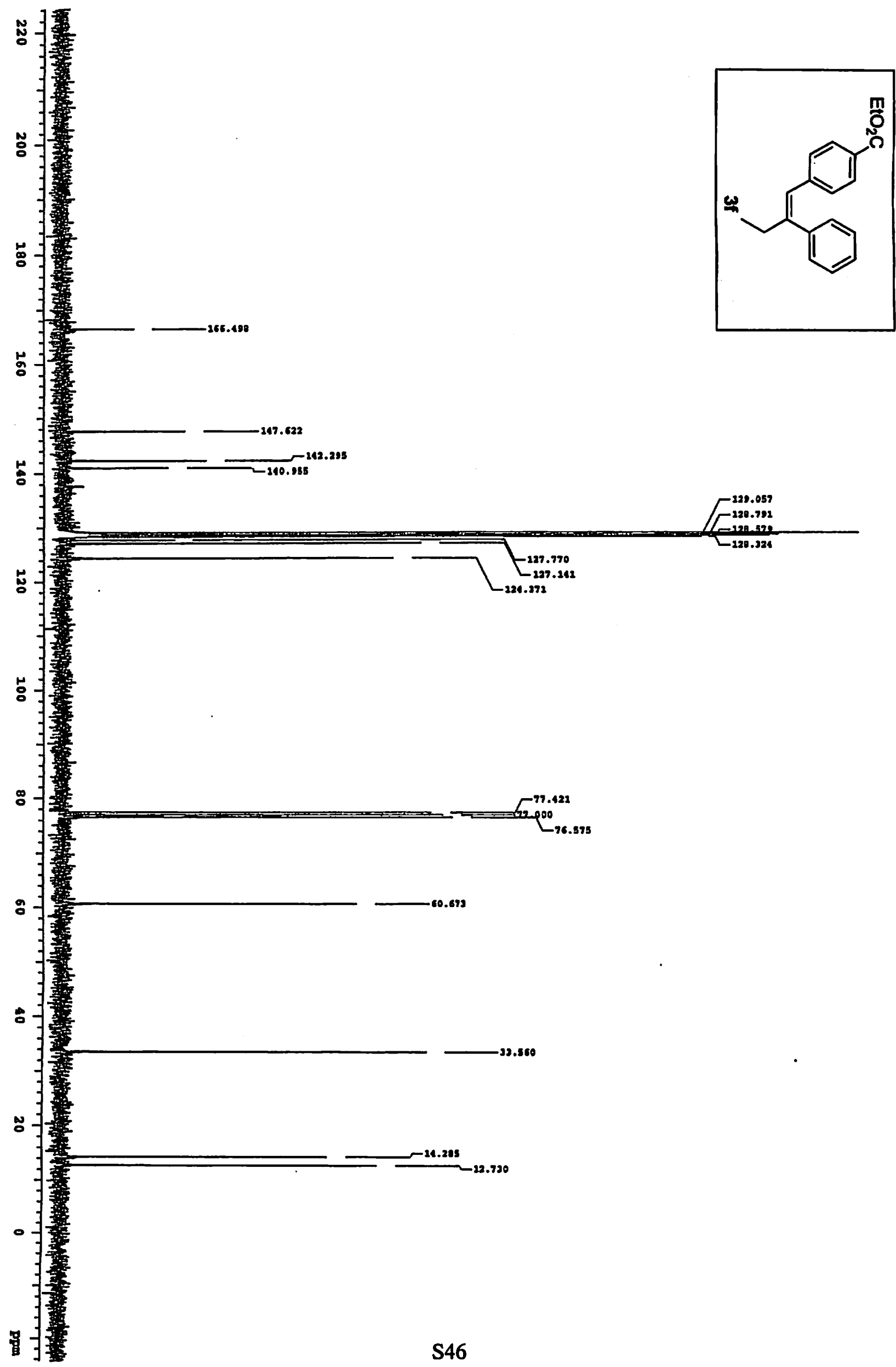




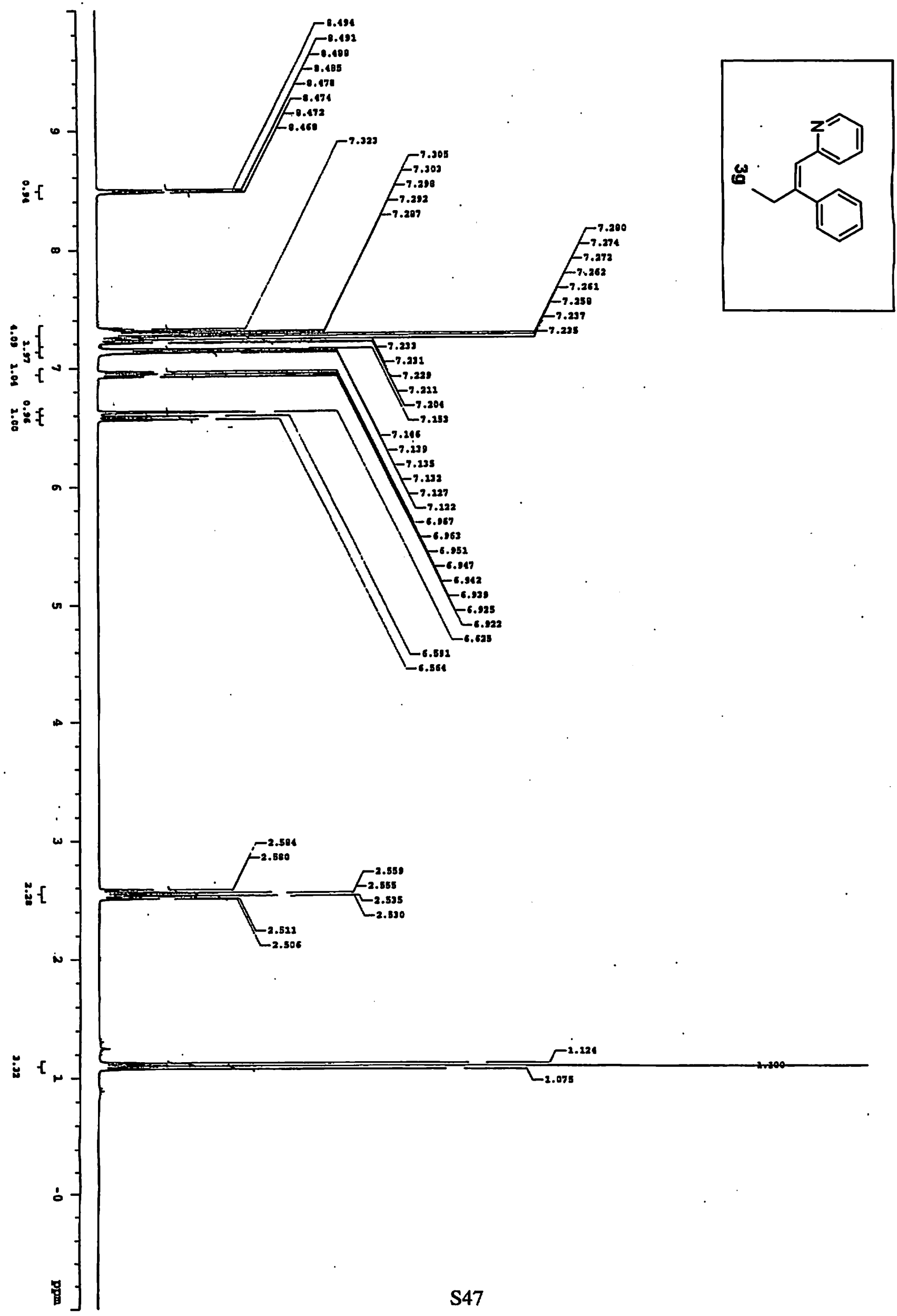




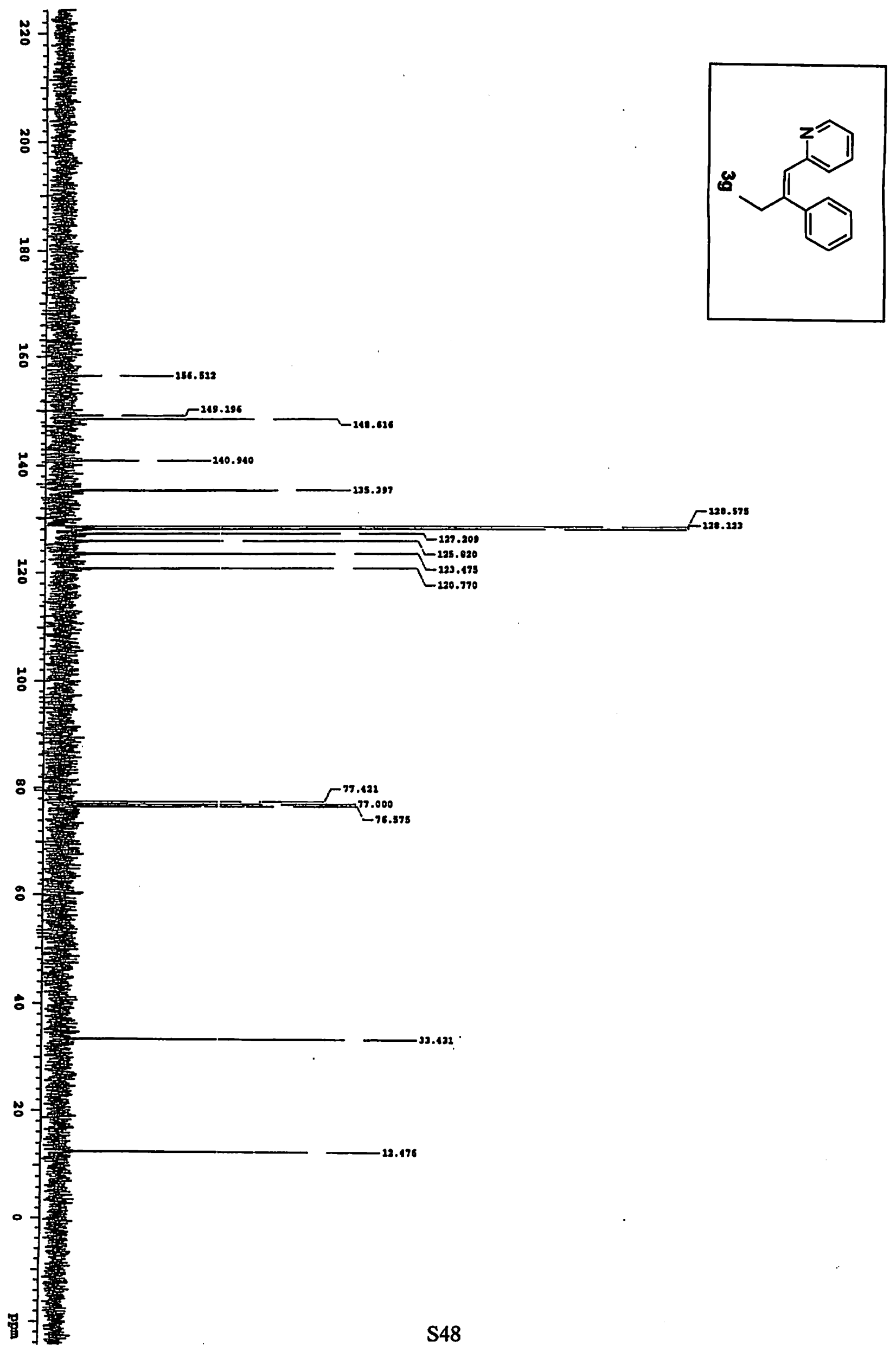




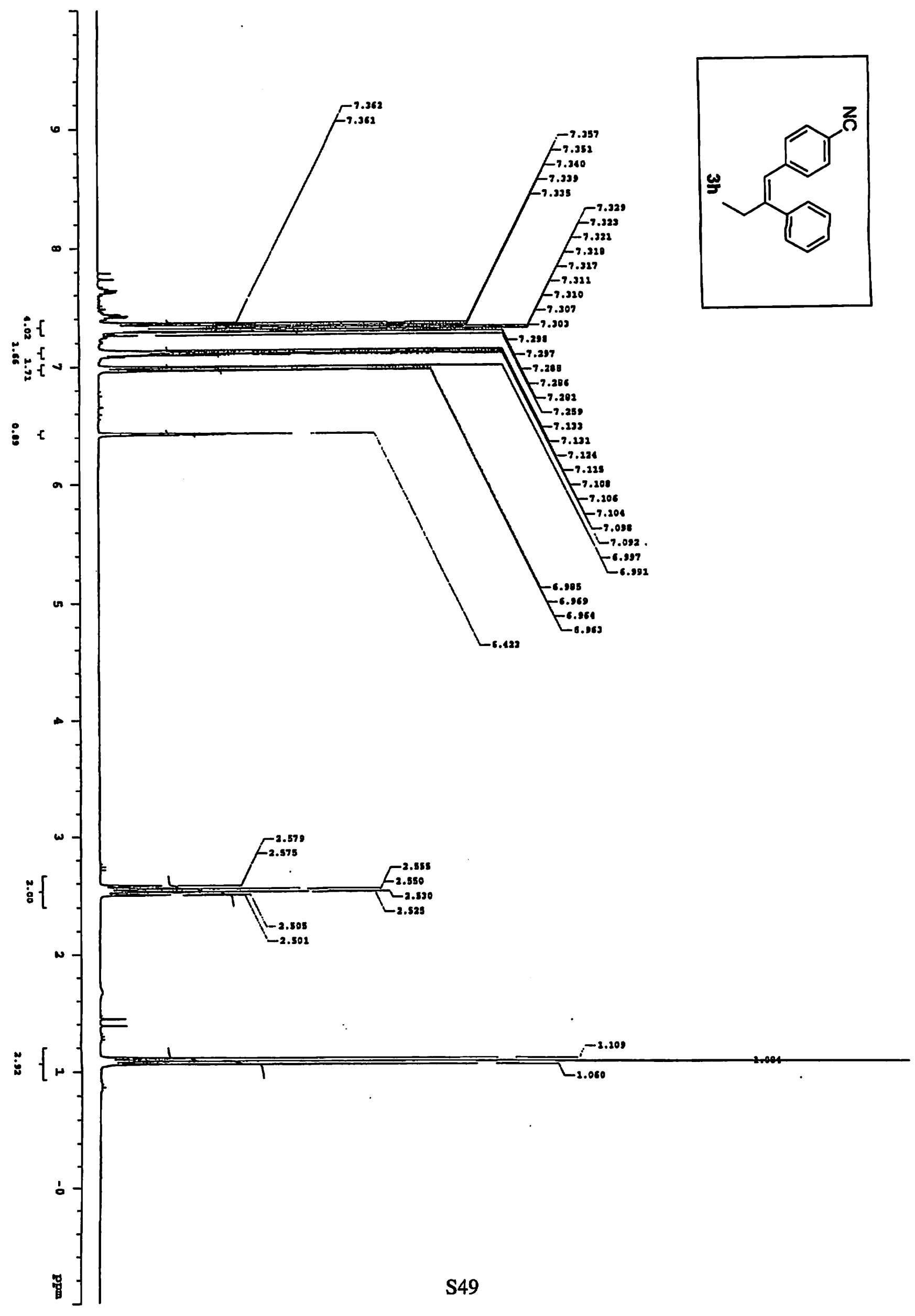




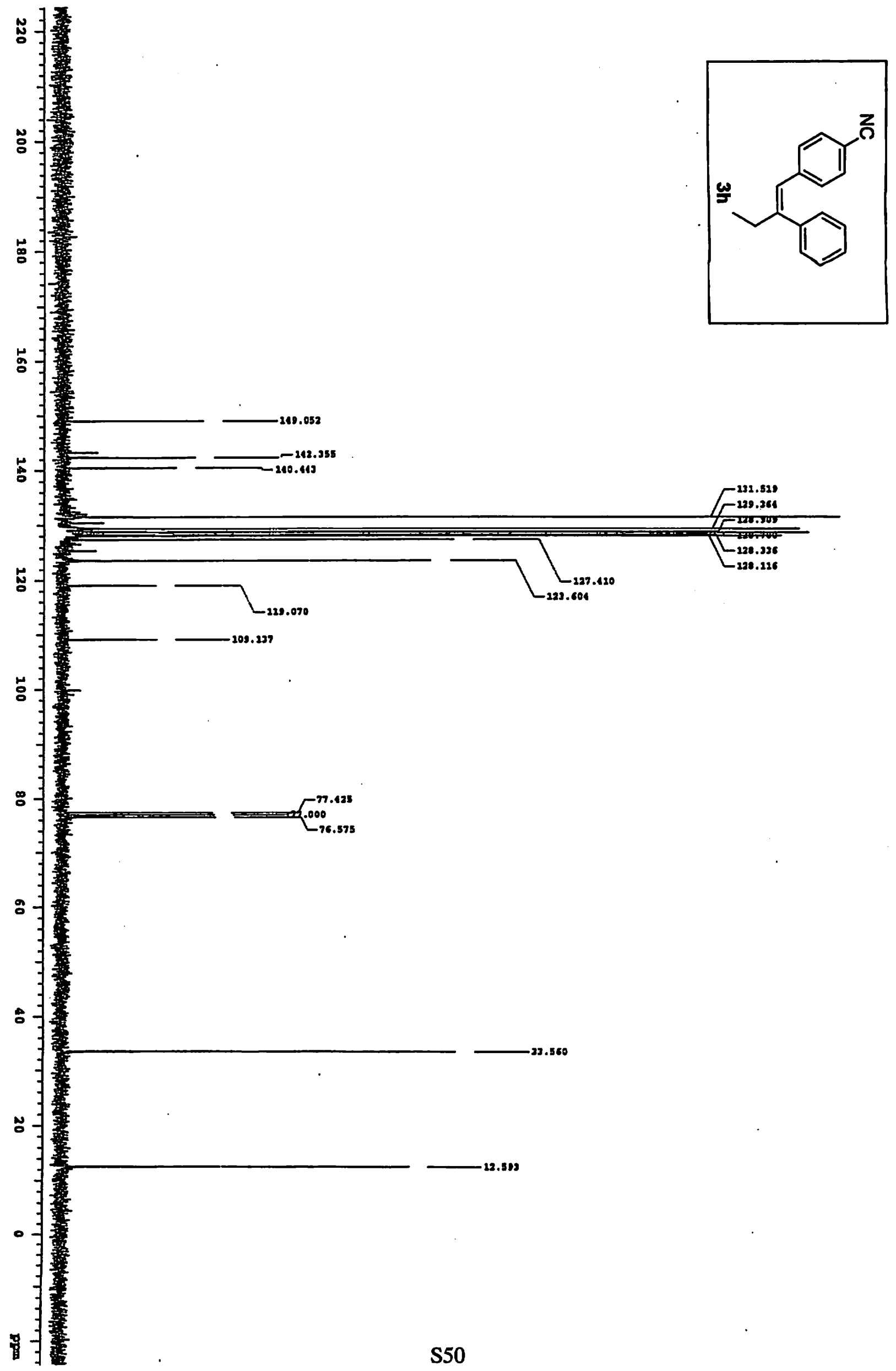




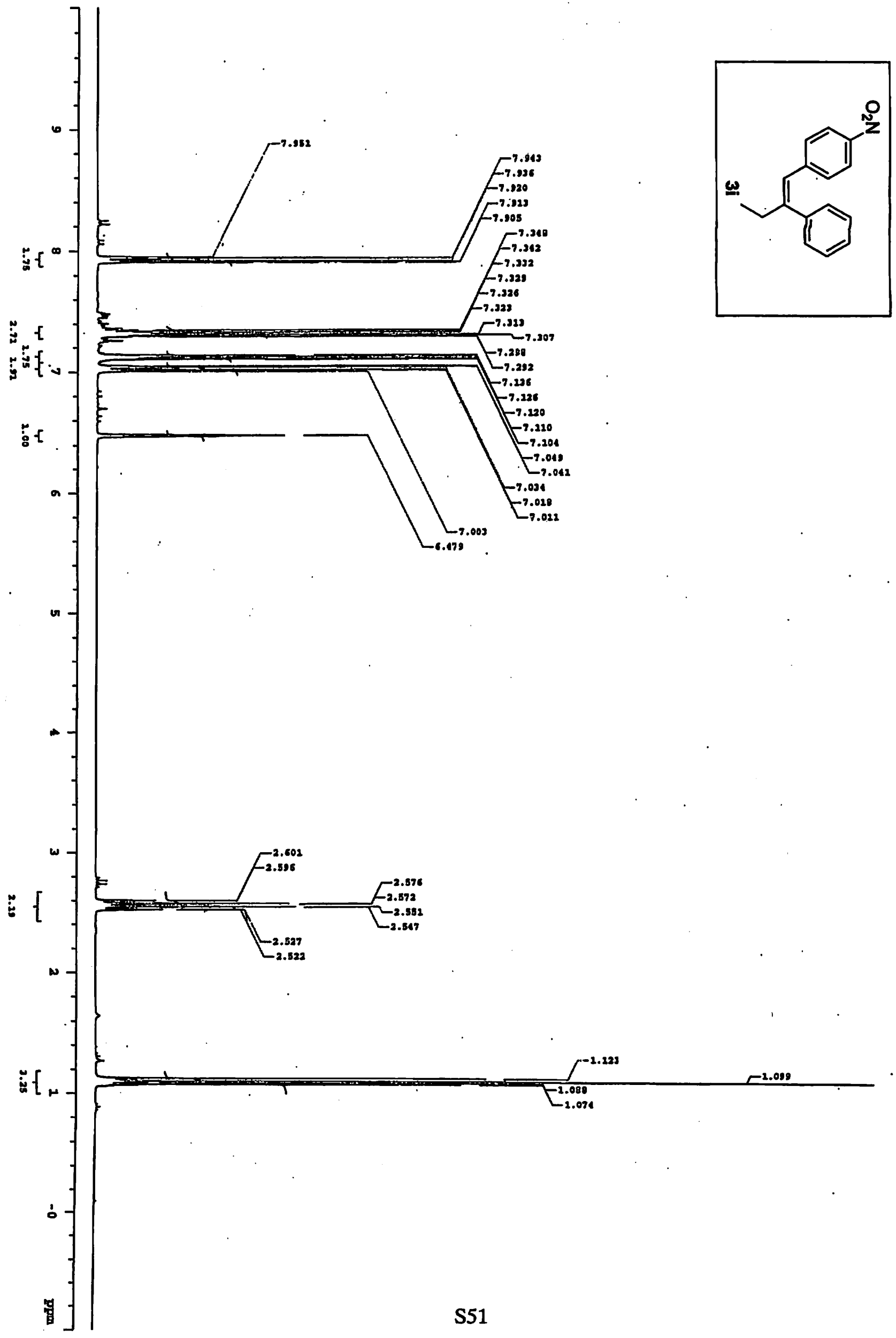




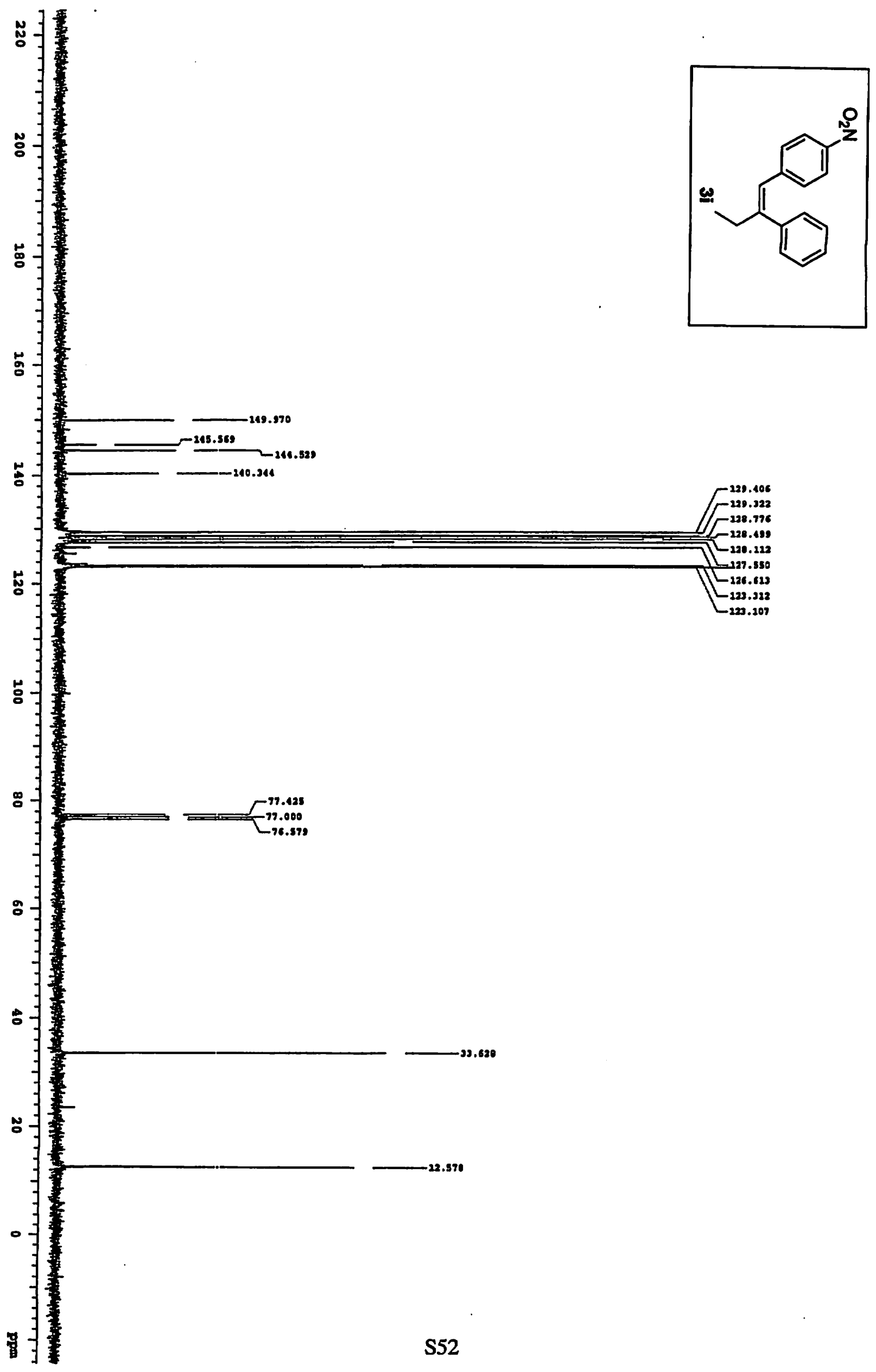




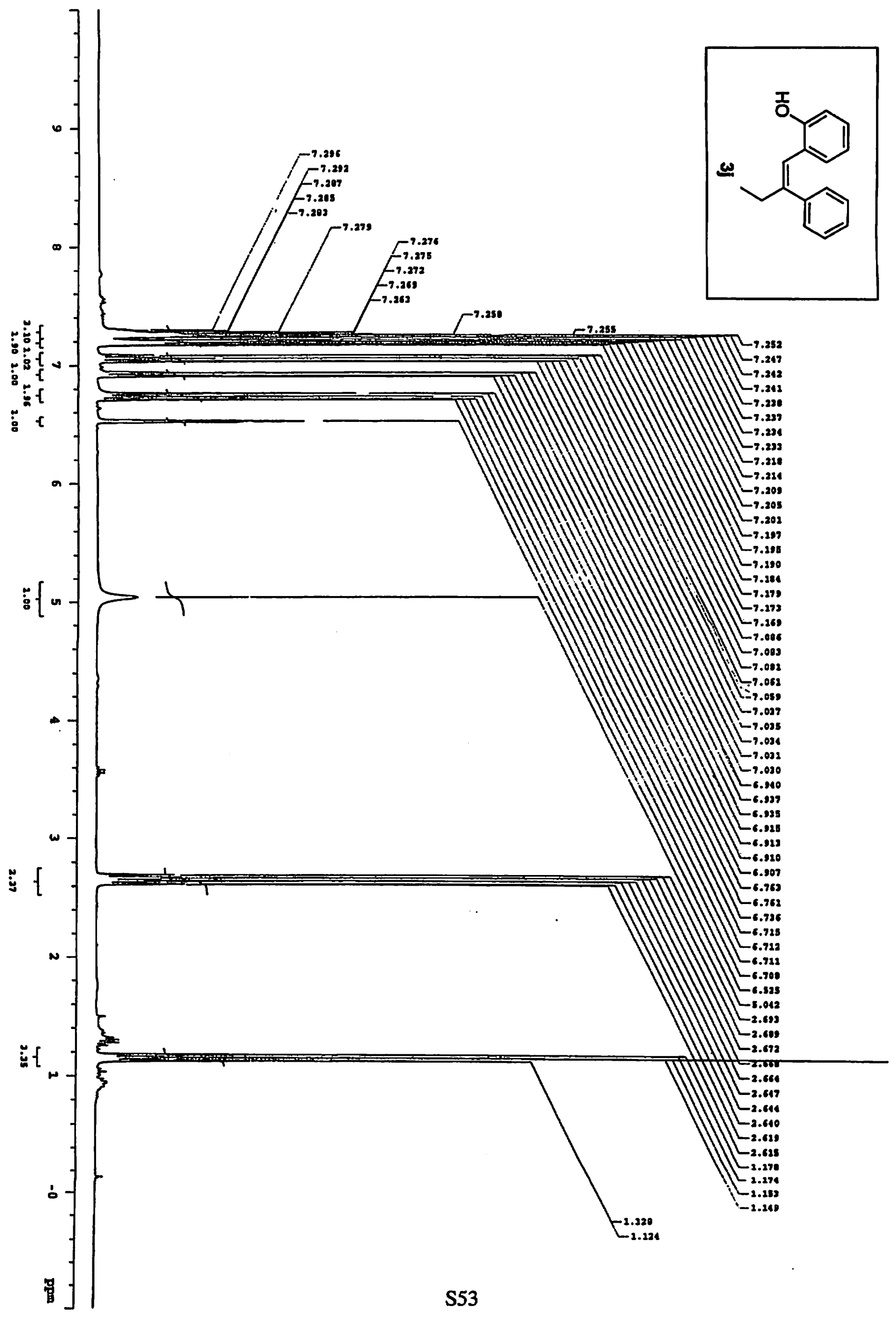




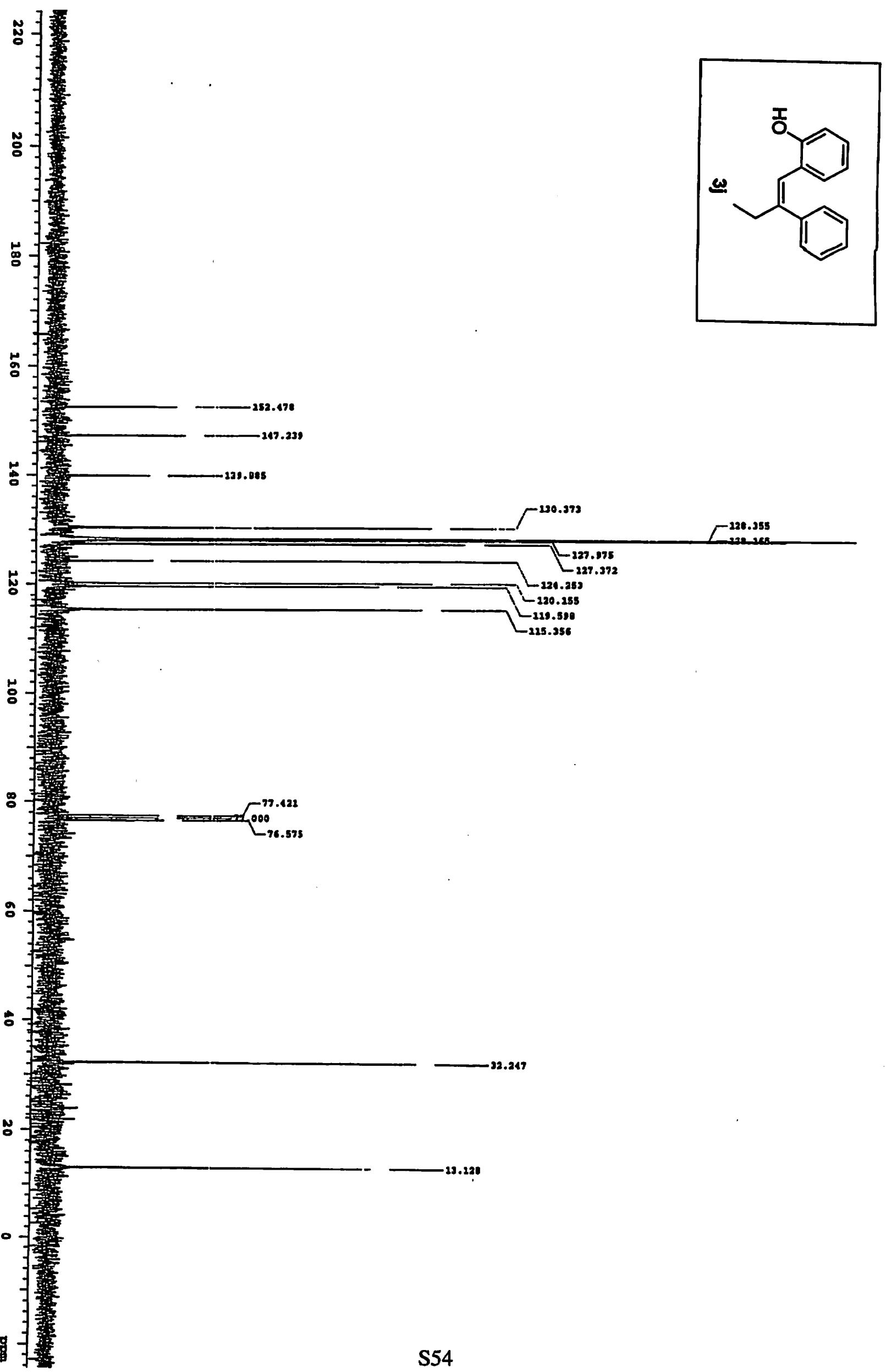




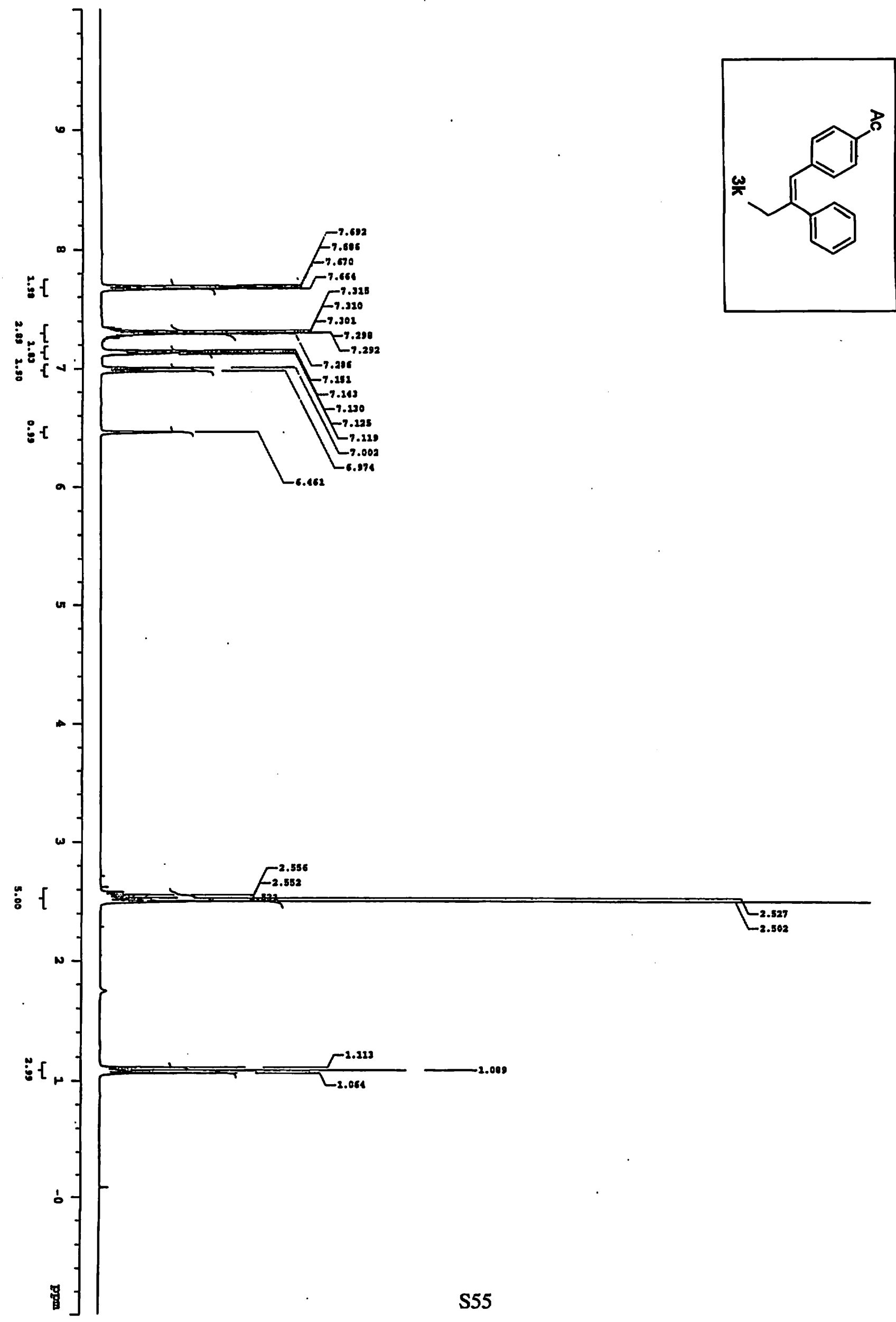




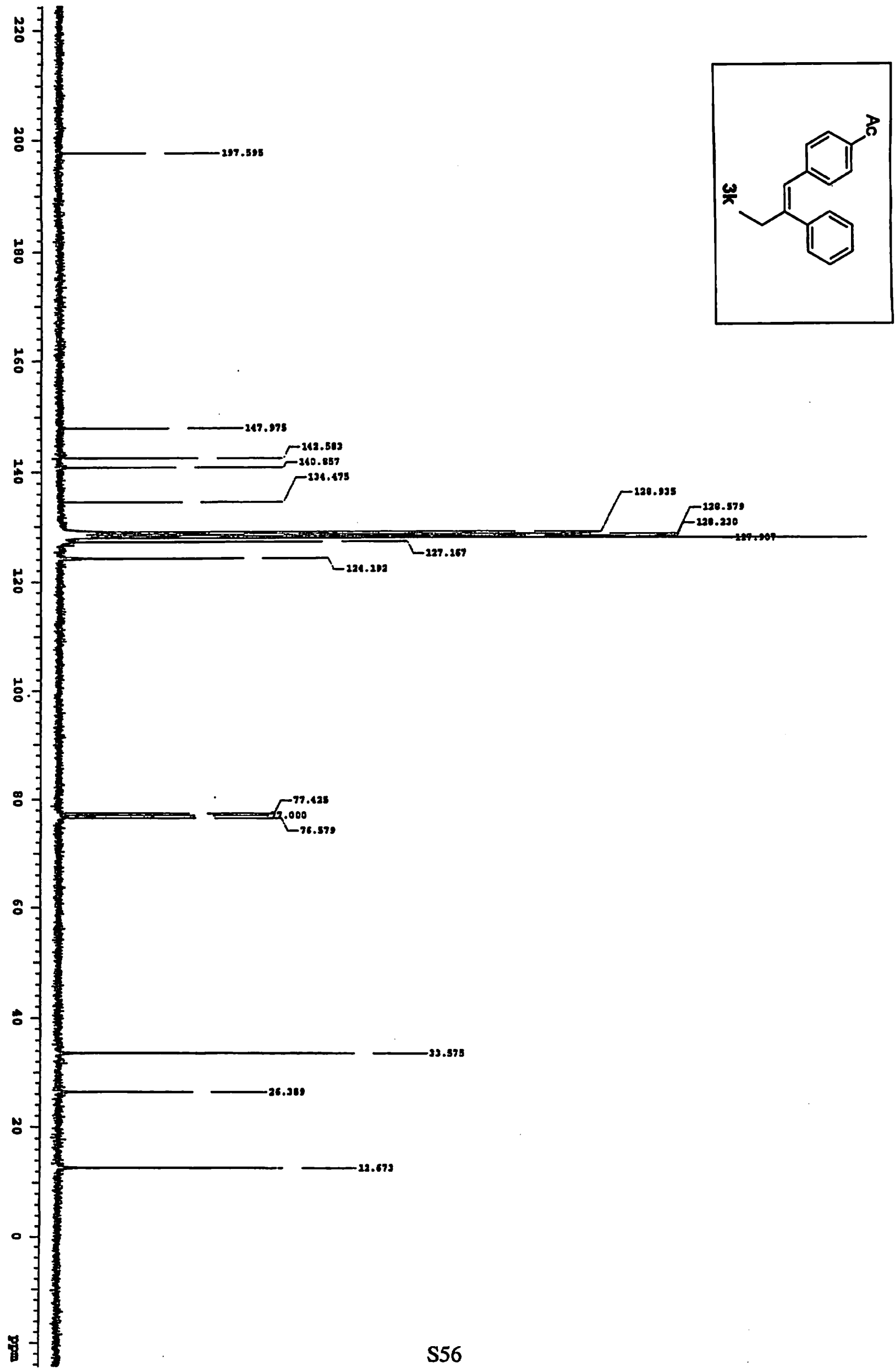




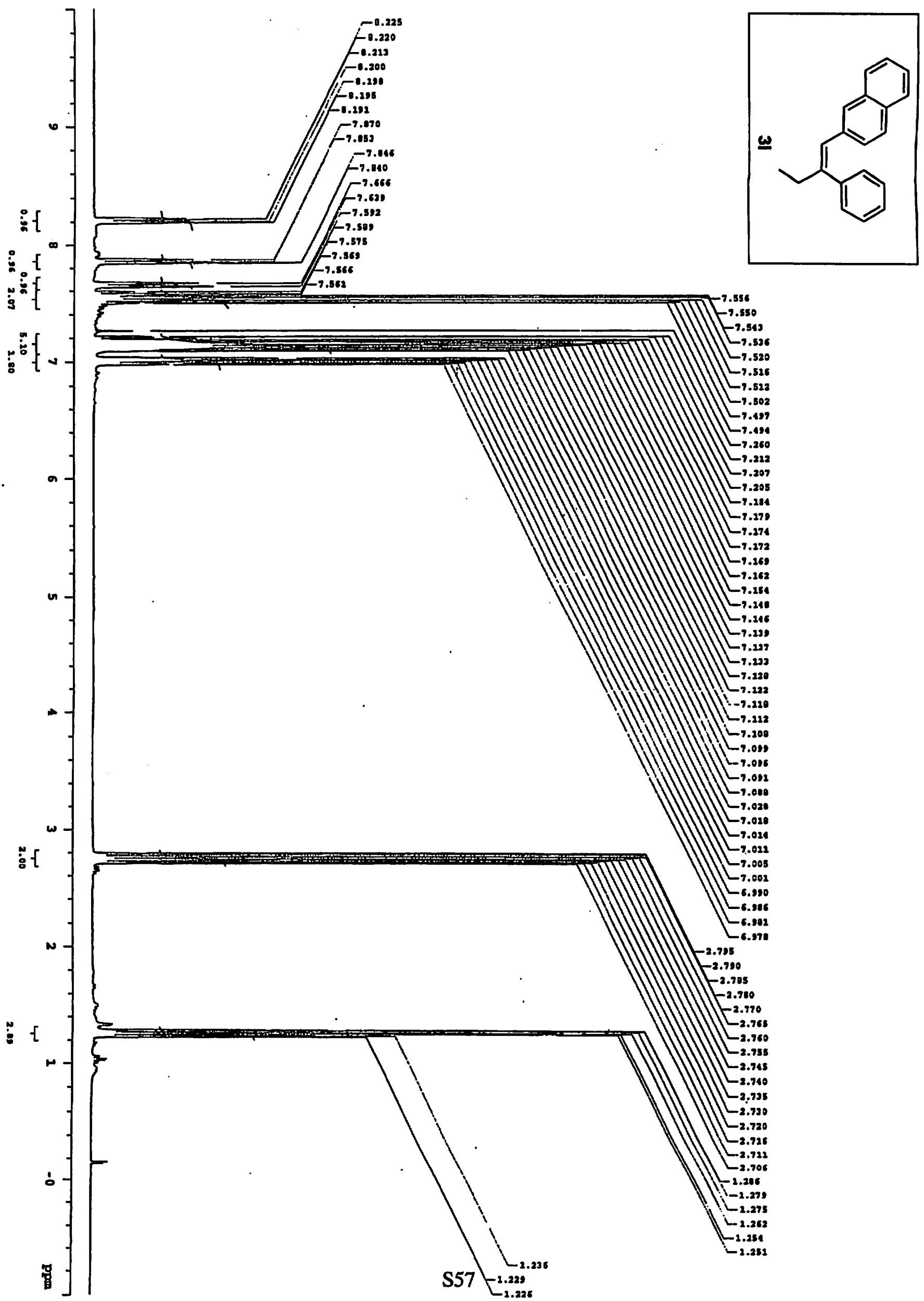




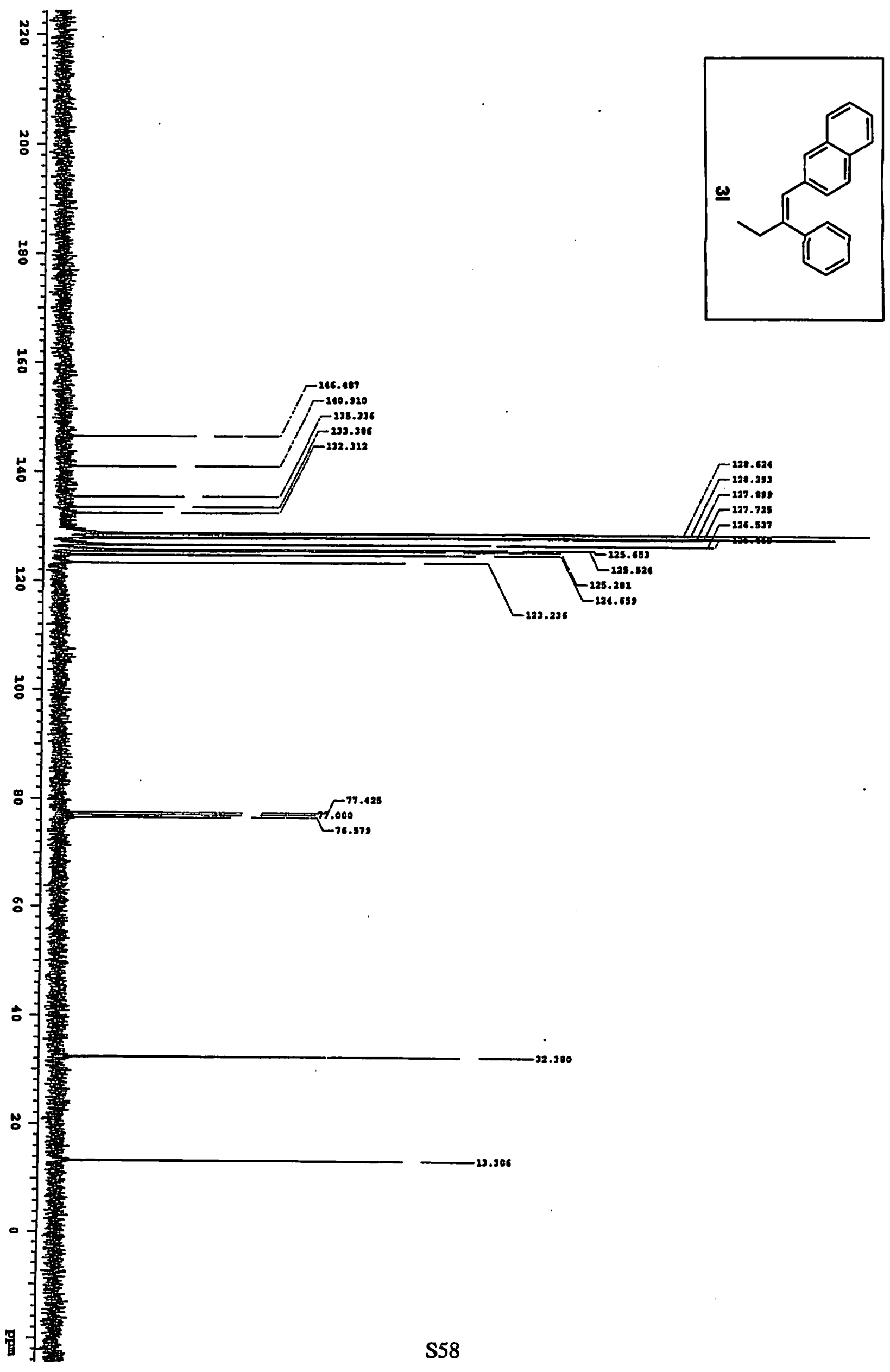




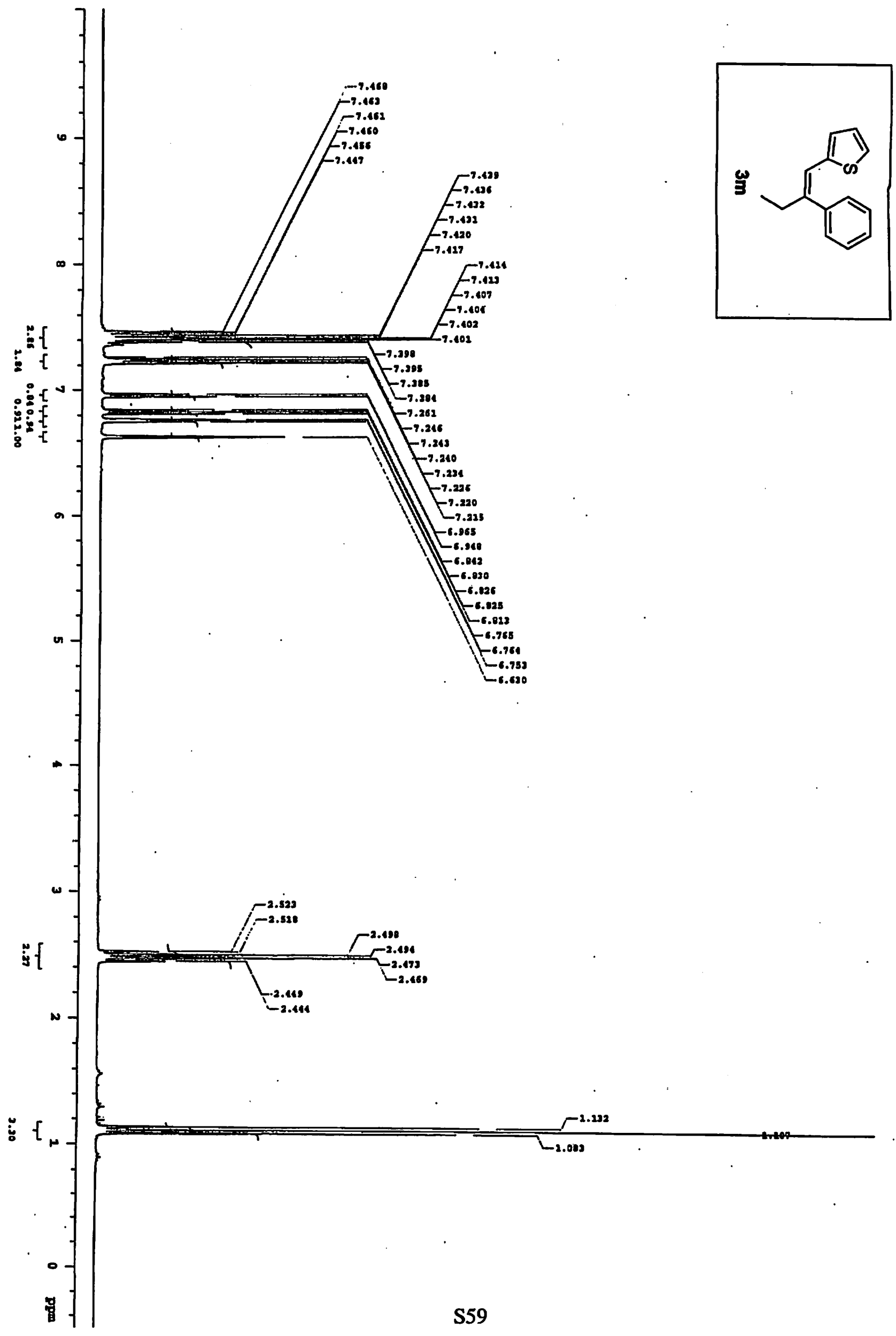




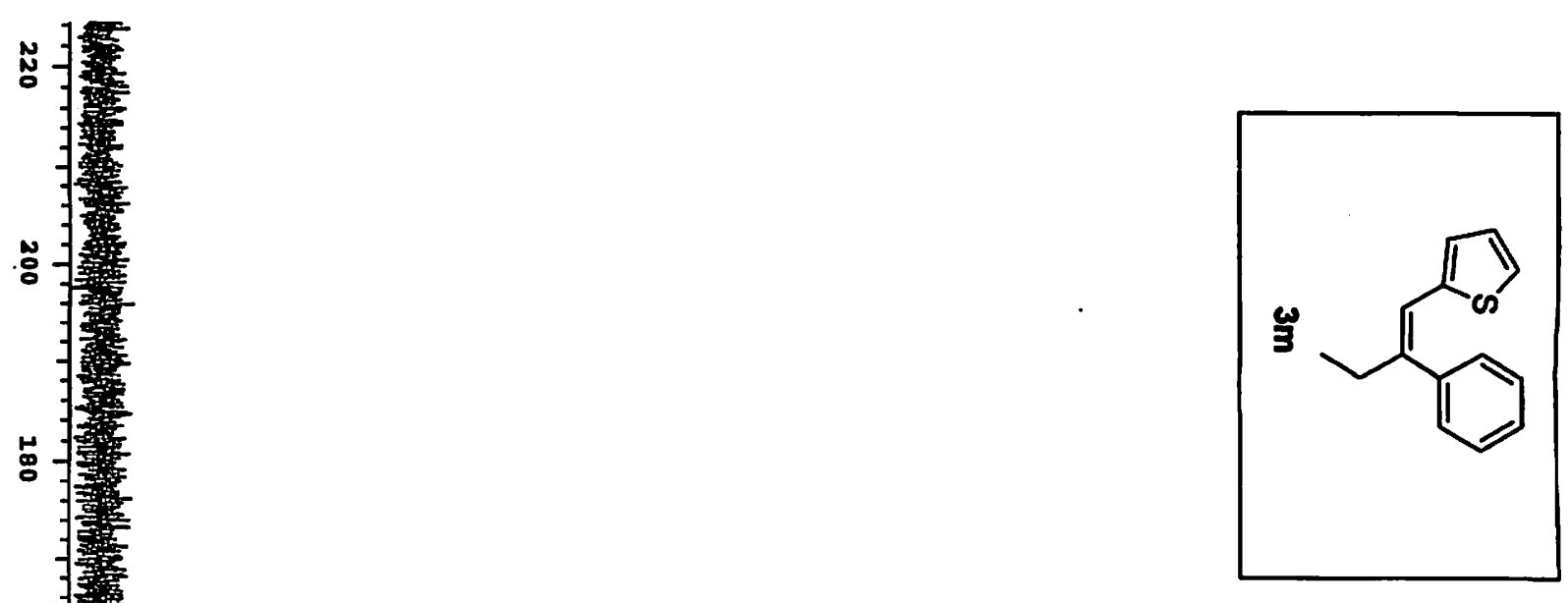

옹

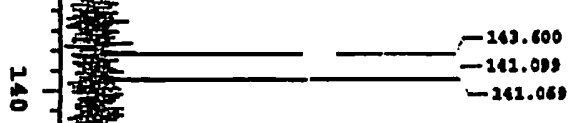

$-220.070$

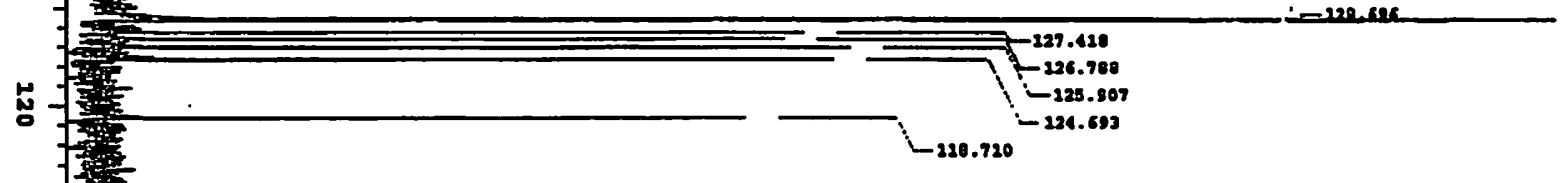

攘

$\Gamma^{77.428}$

$-76.578$

:

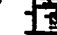

s-
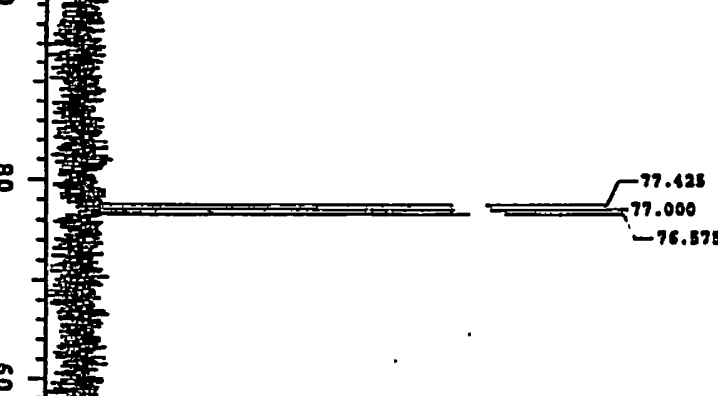

. 


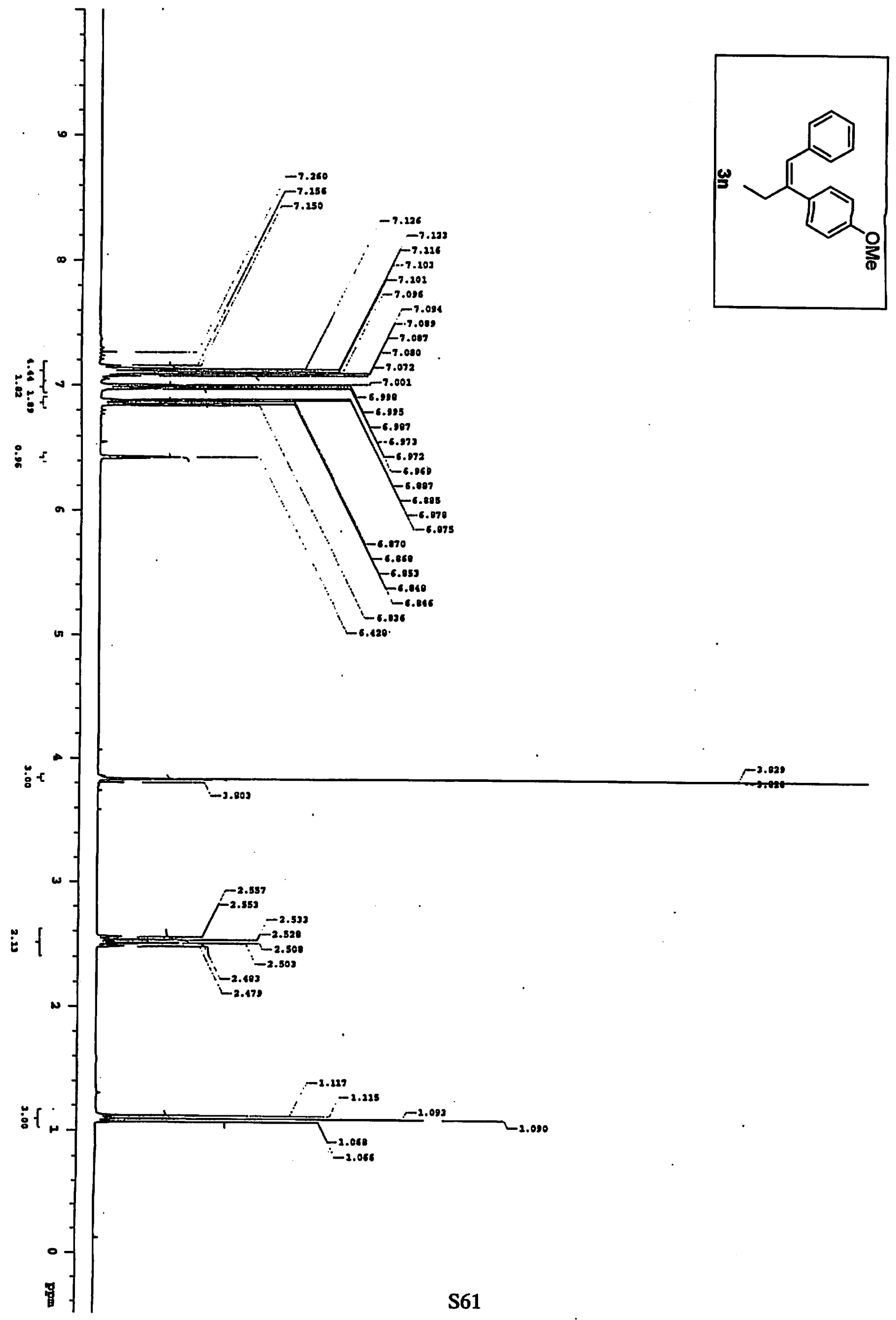




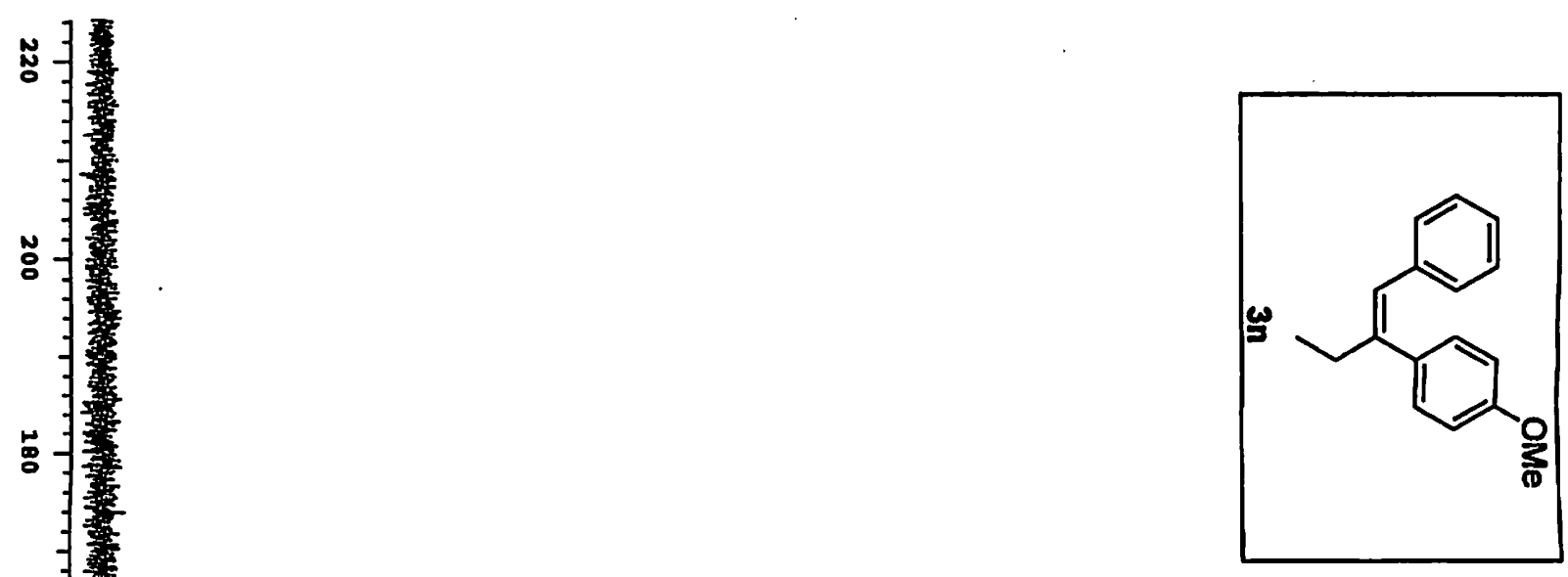

응-

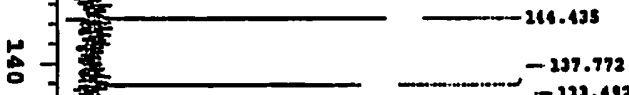

$-231.482$

$\Gamma_{-220.963}^{228.628}$

$-27.425$

-76.000
-76.535

s -

.

:

용

:

\&

:

-1

-

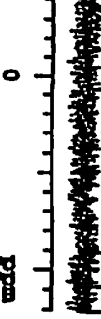

$-22.938$ 


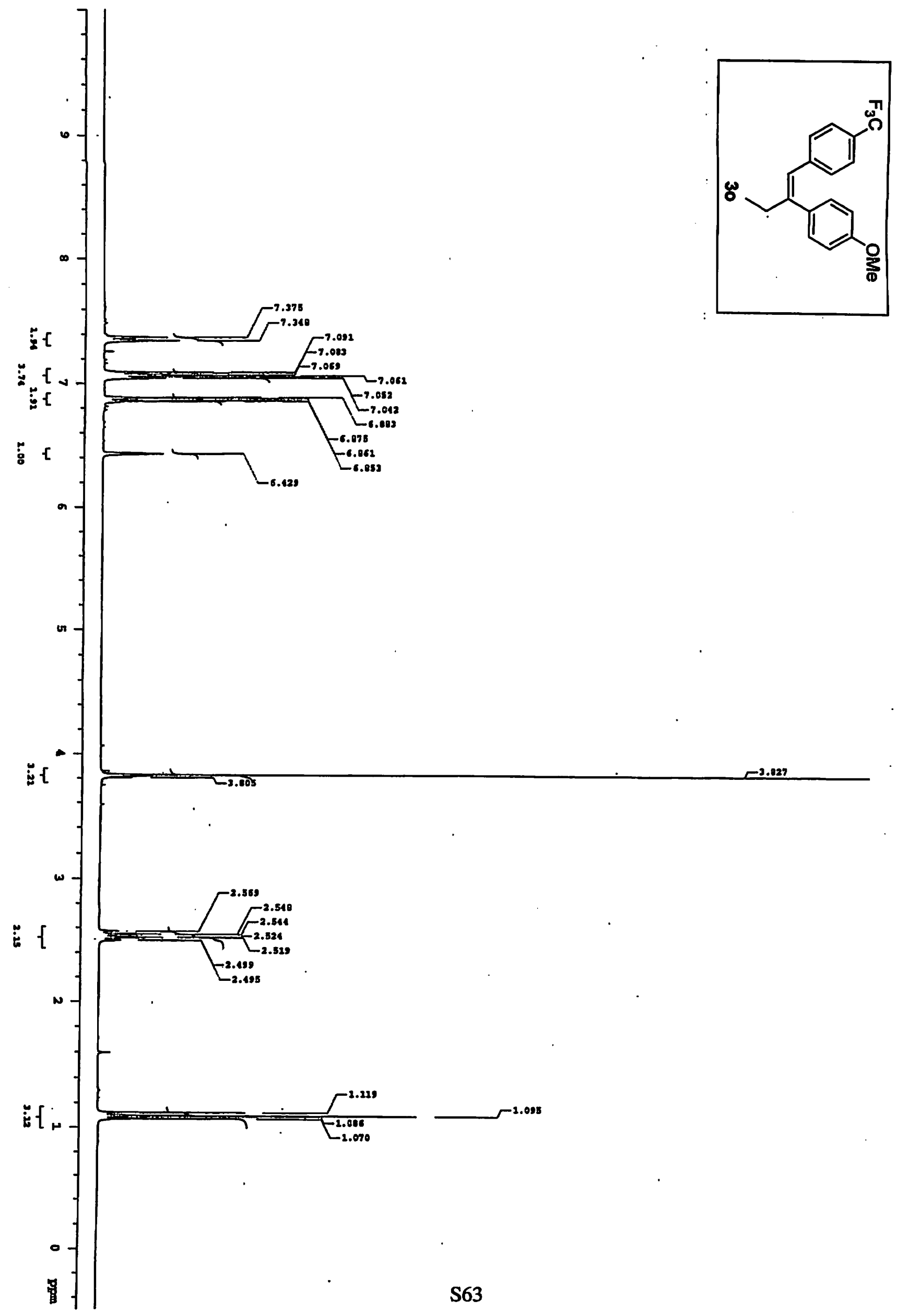




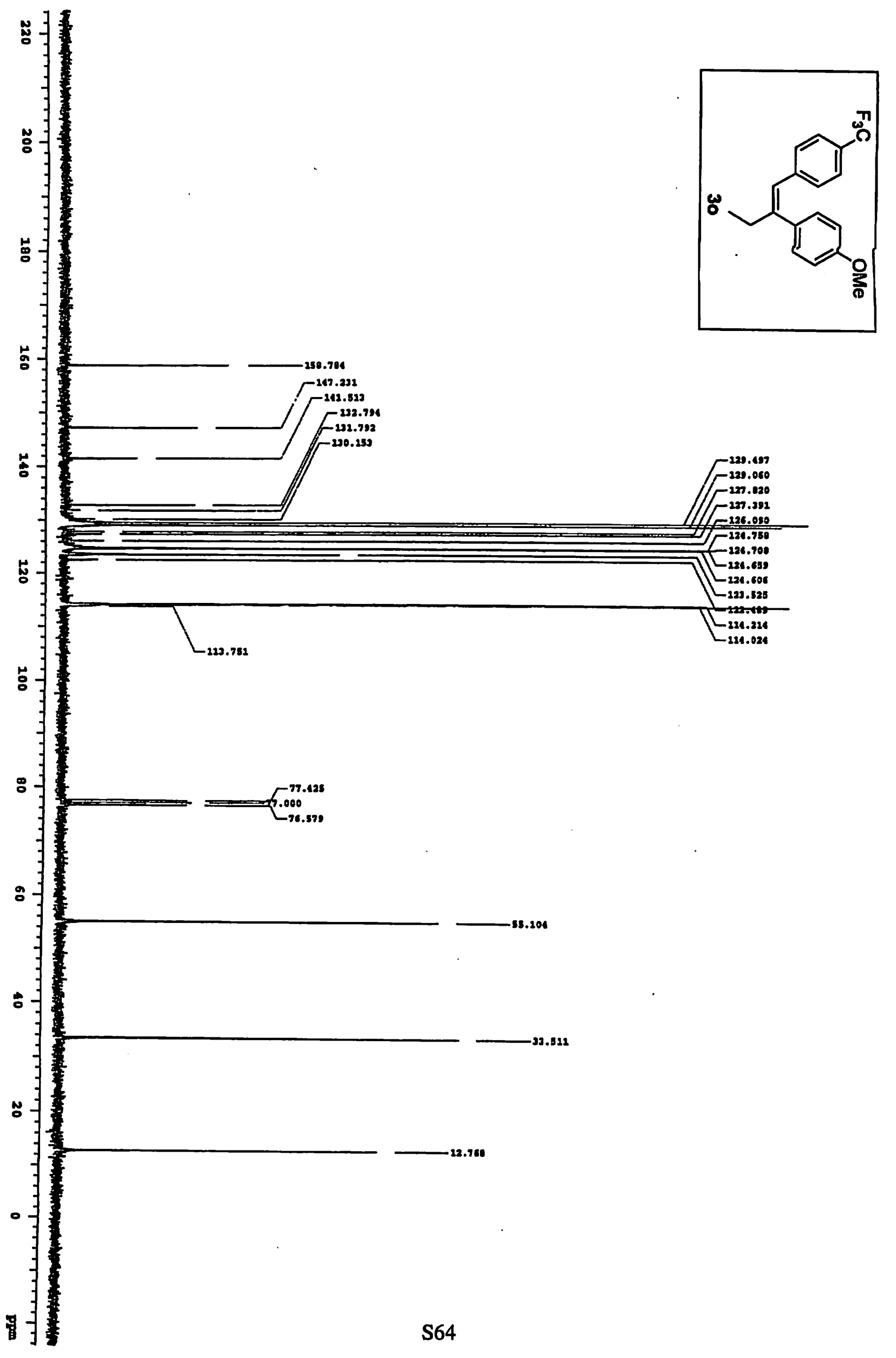




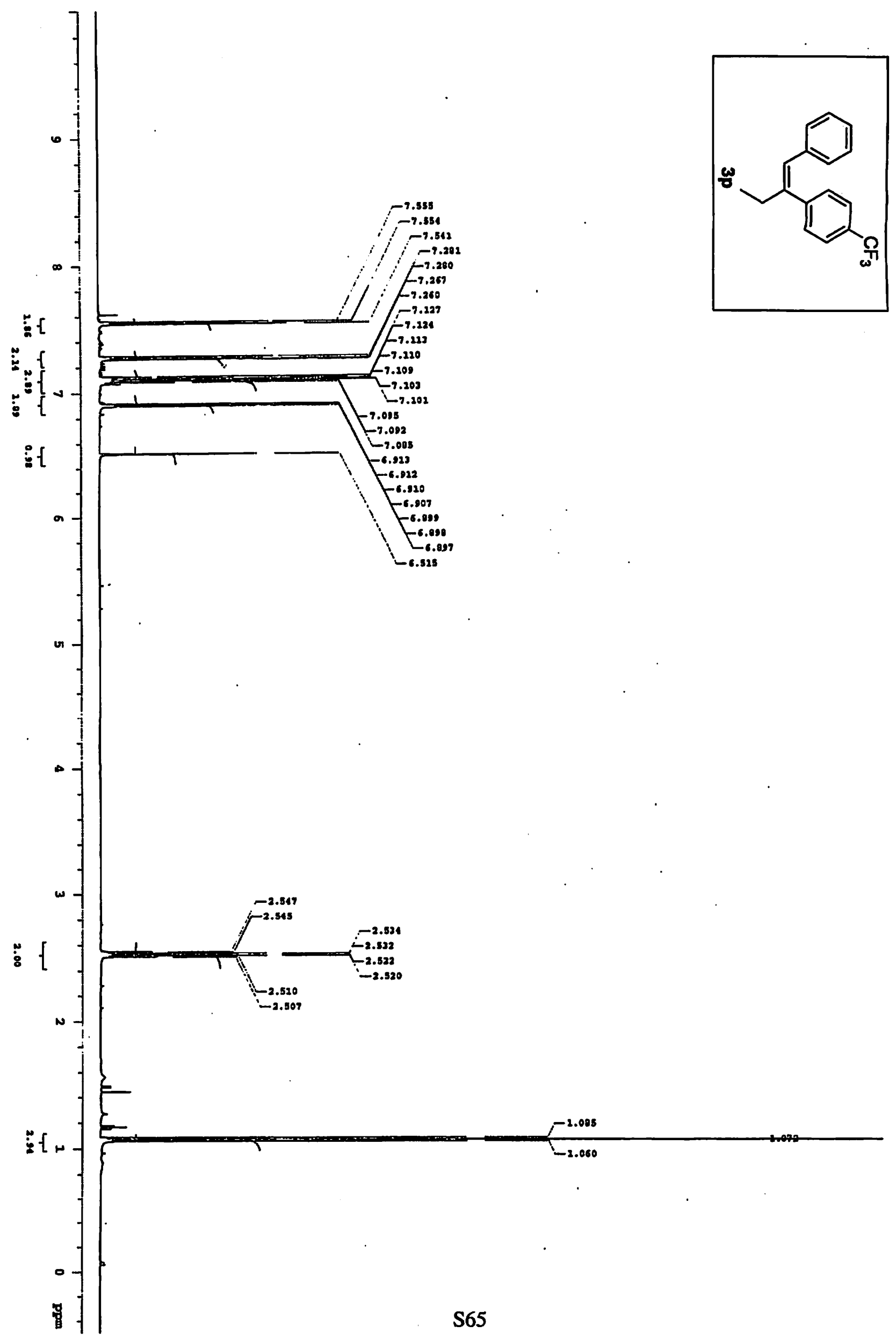




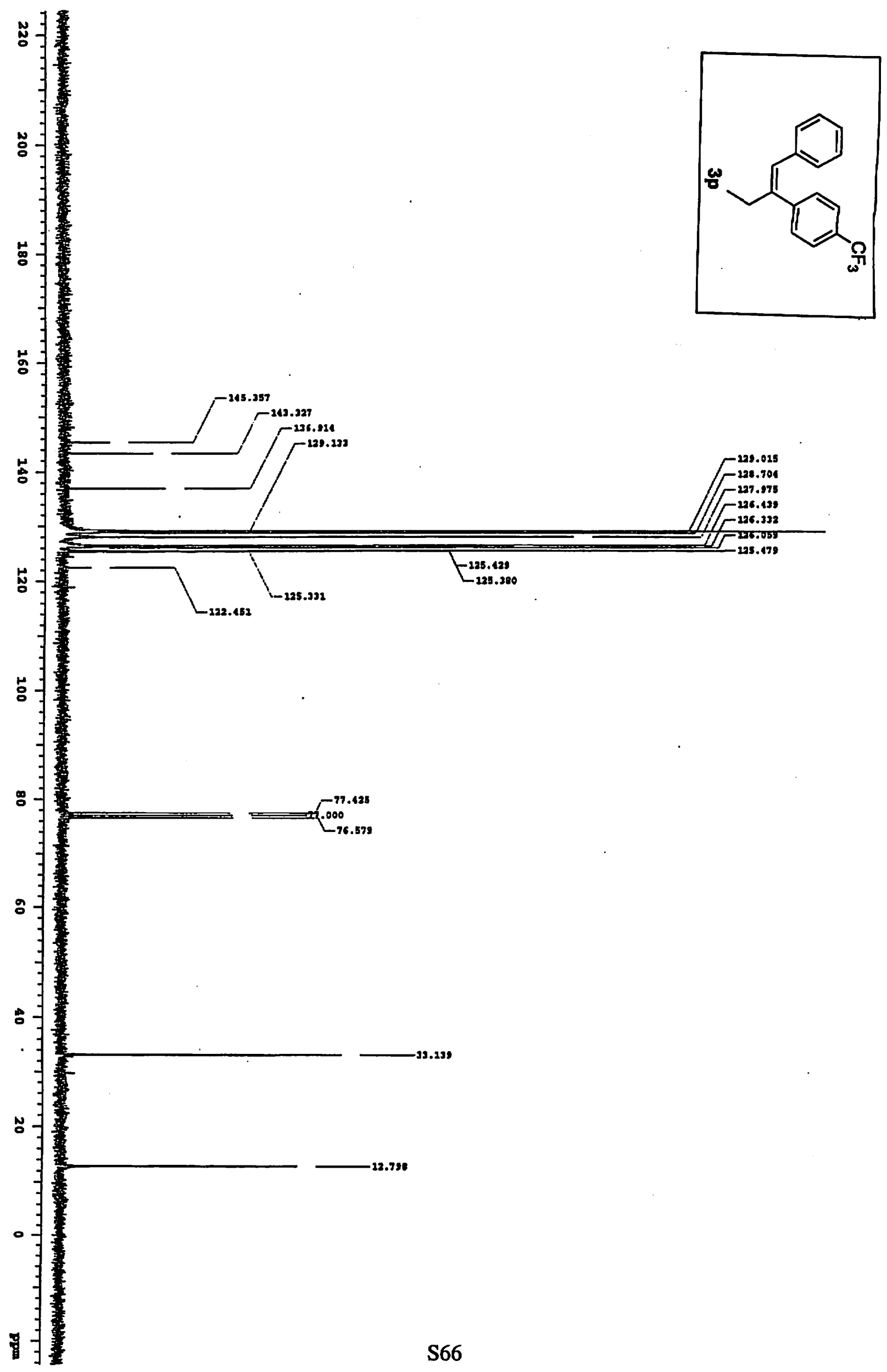




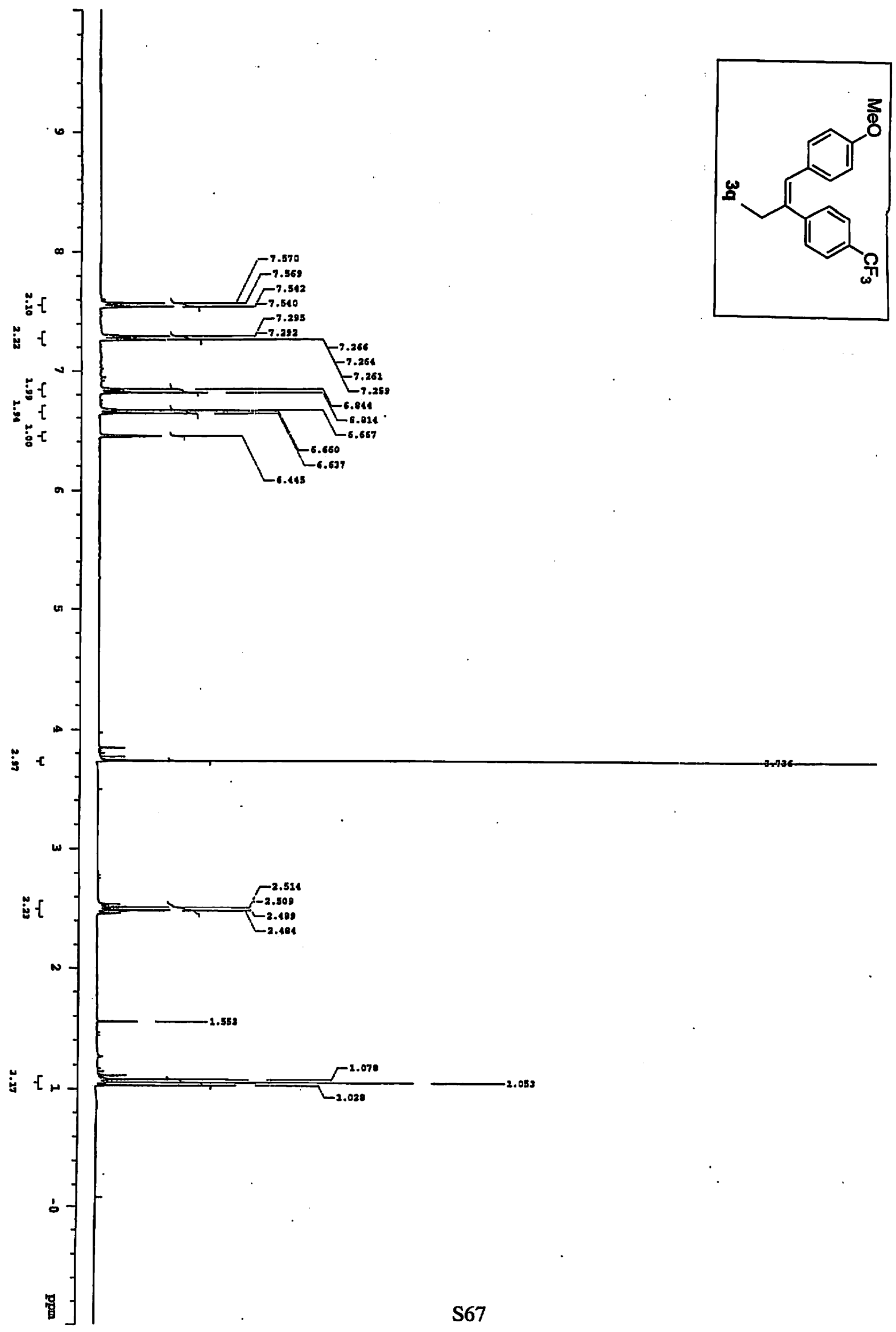




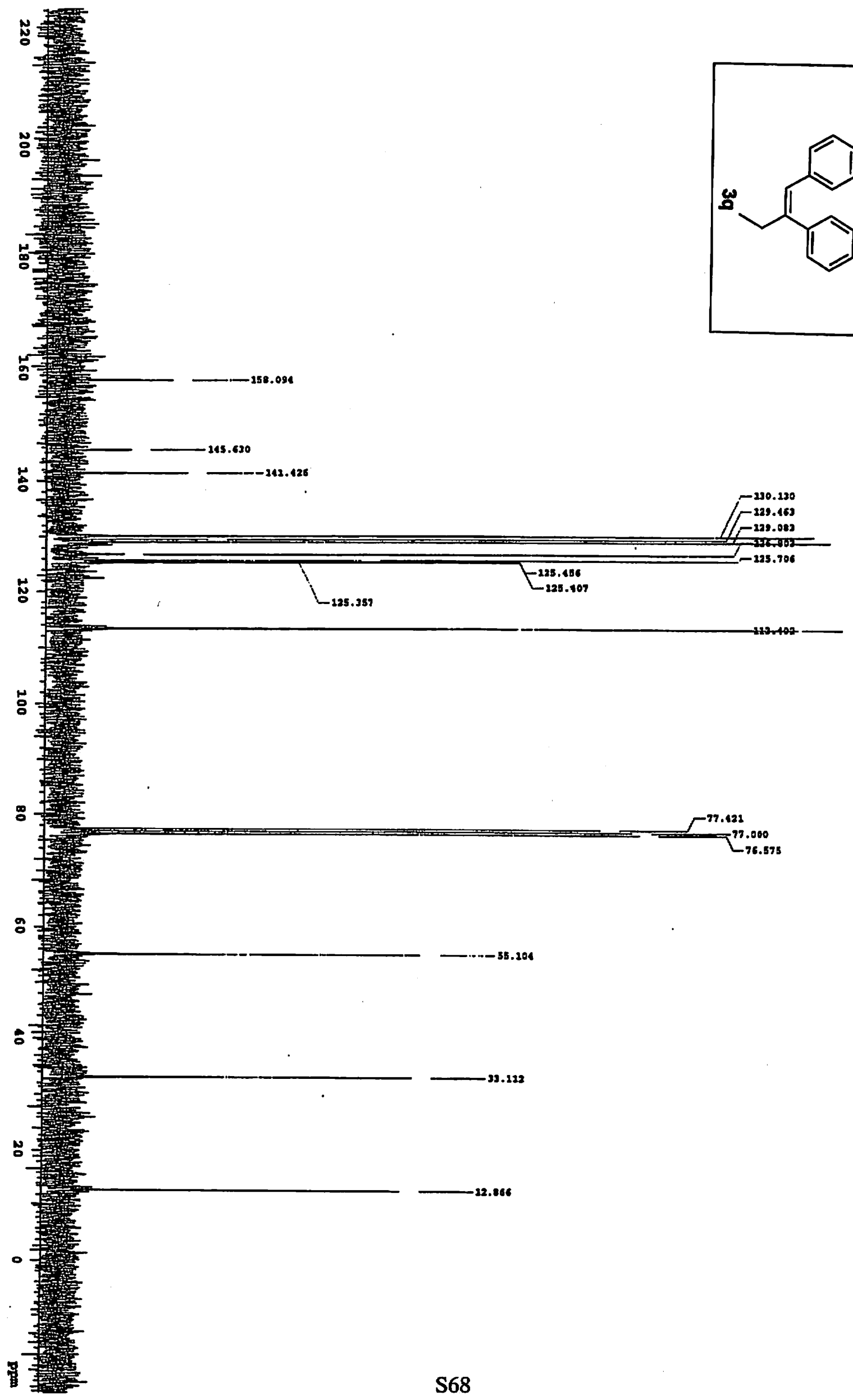




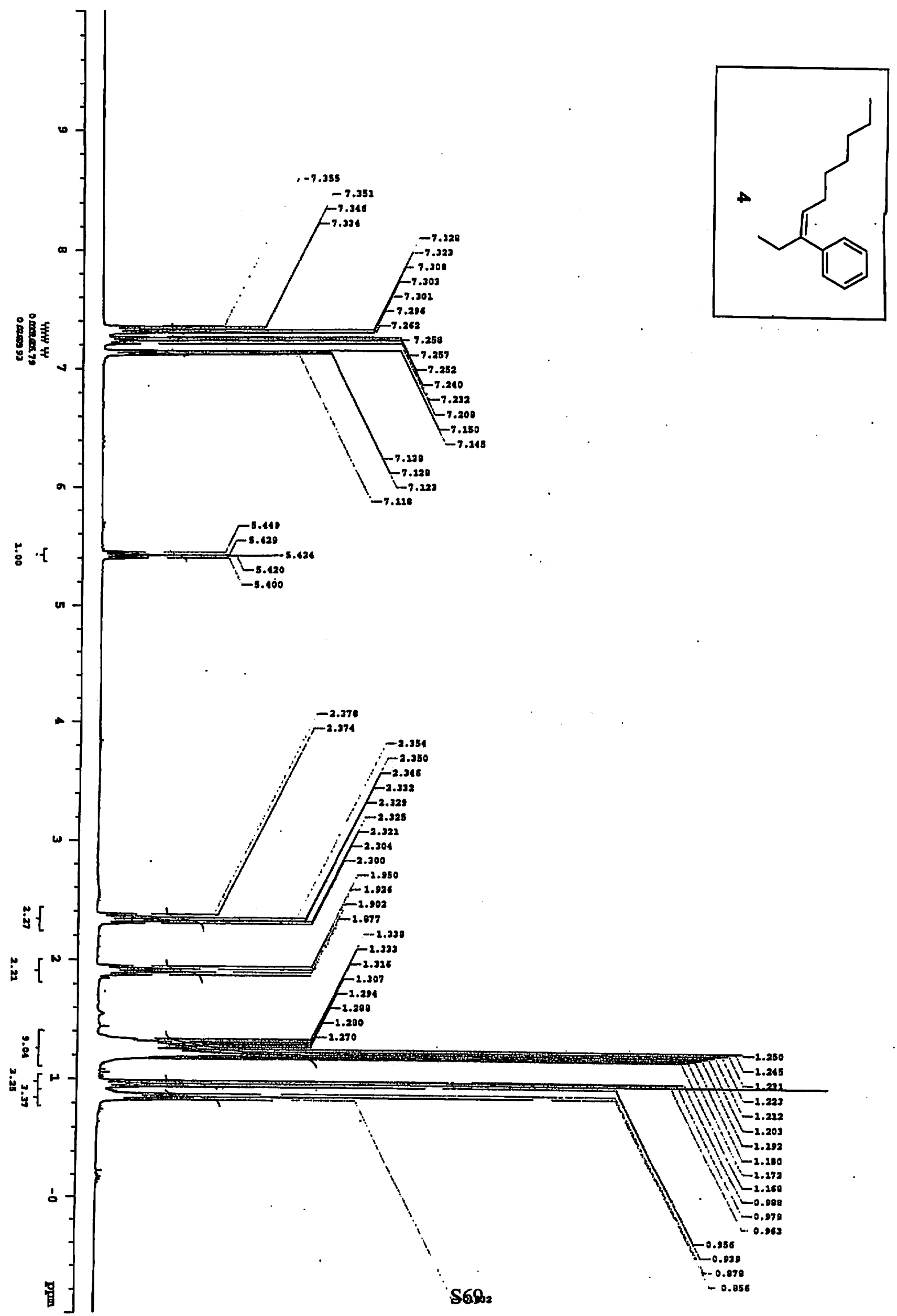




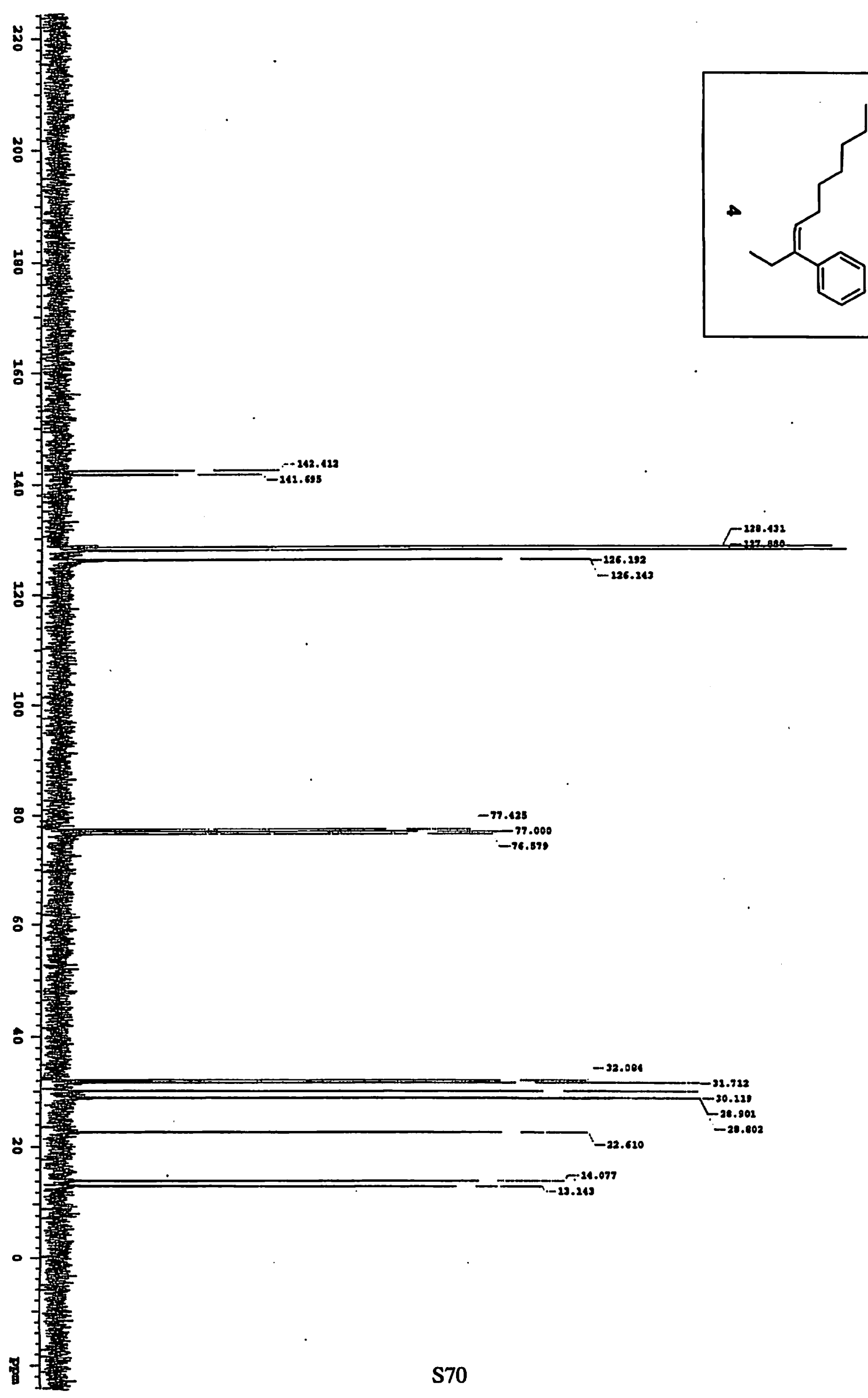




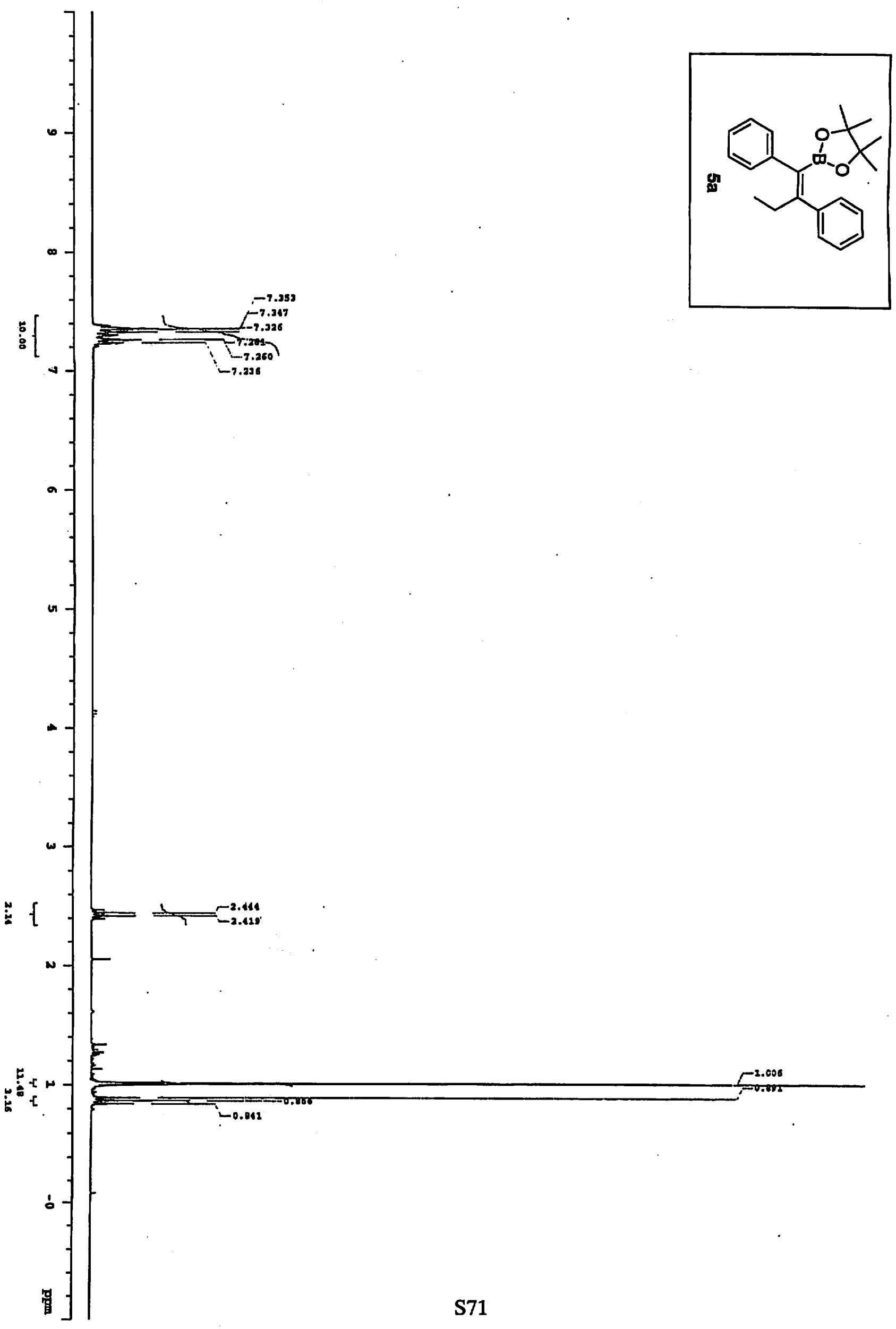




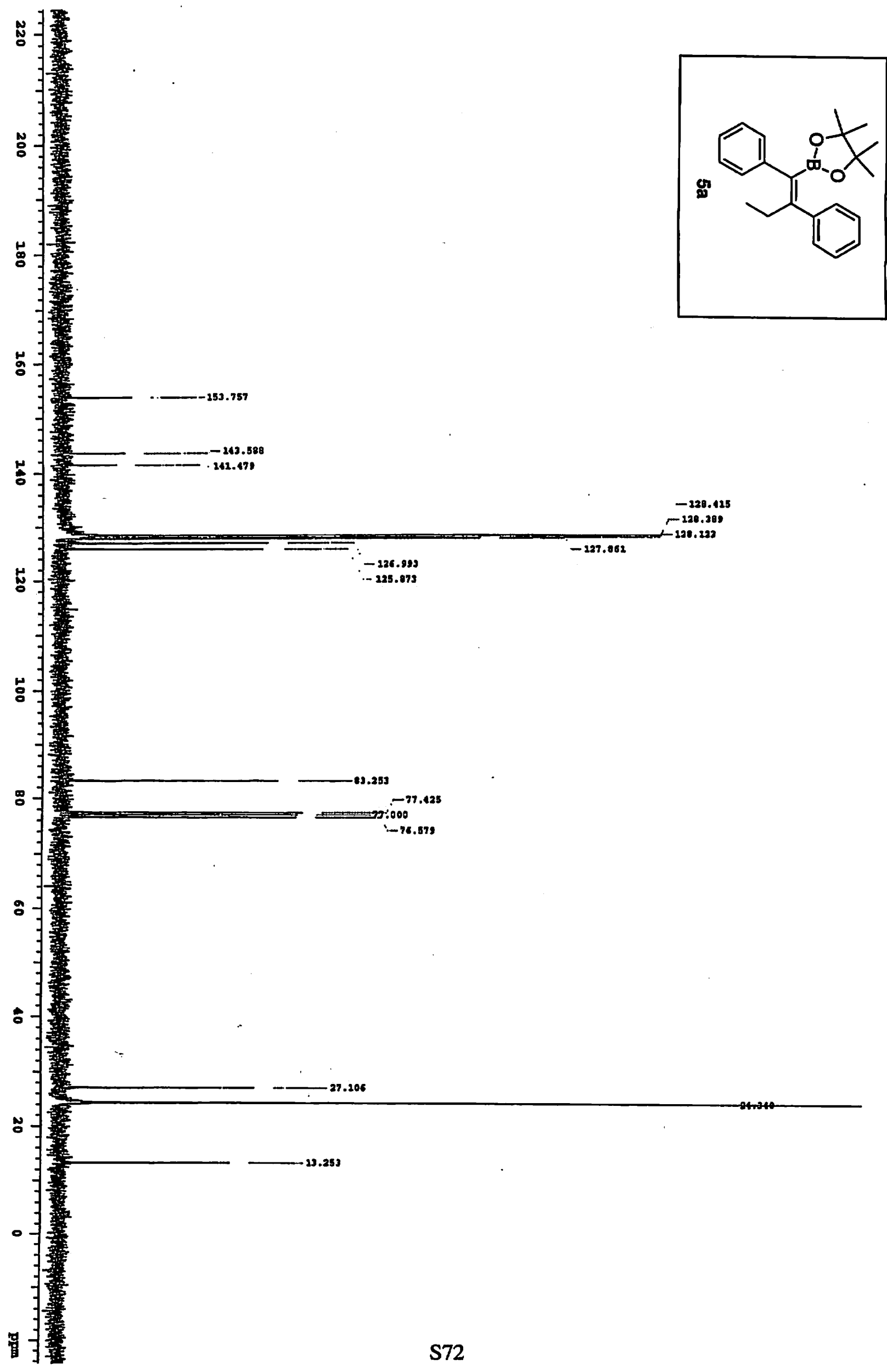




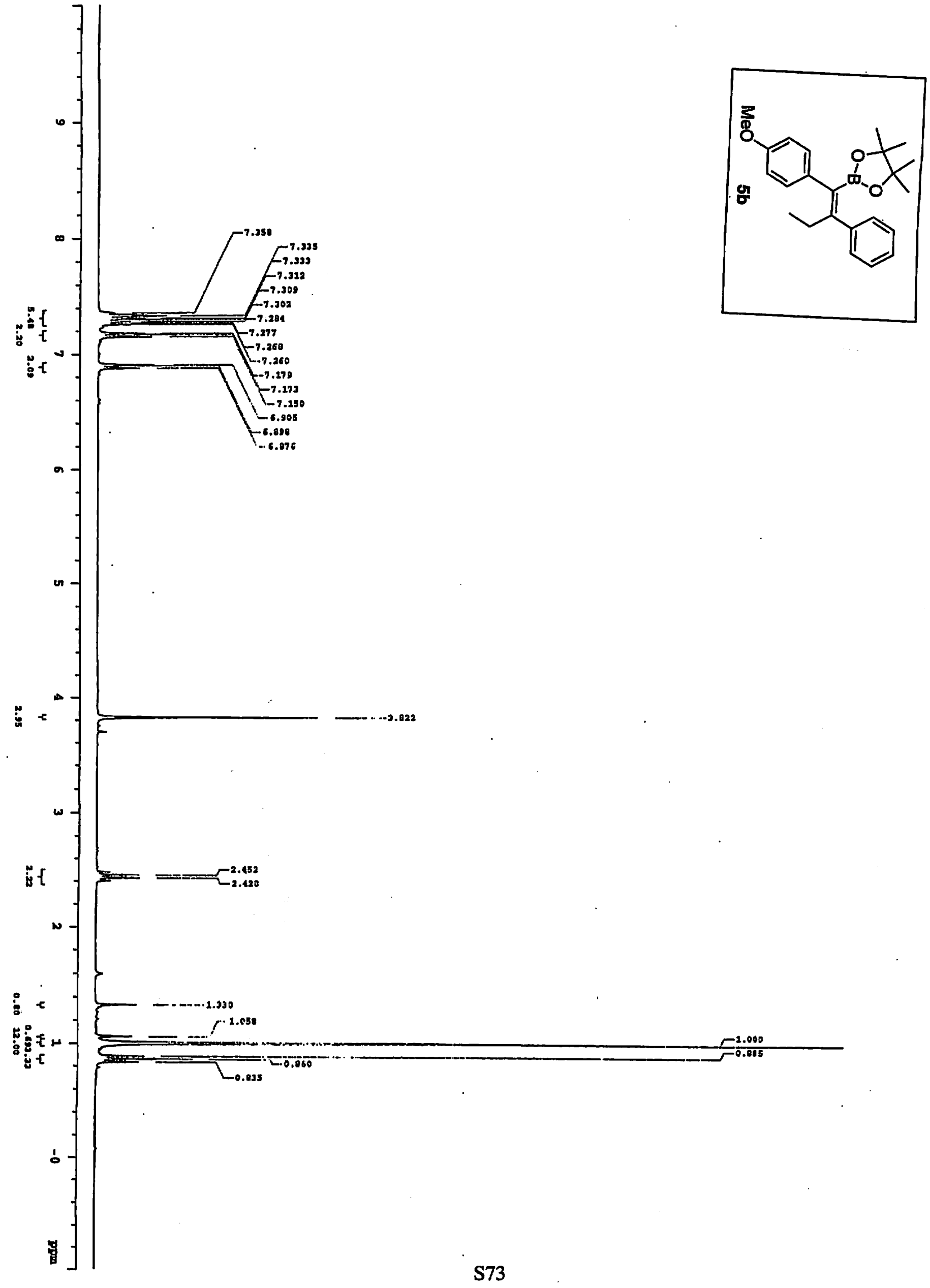




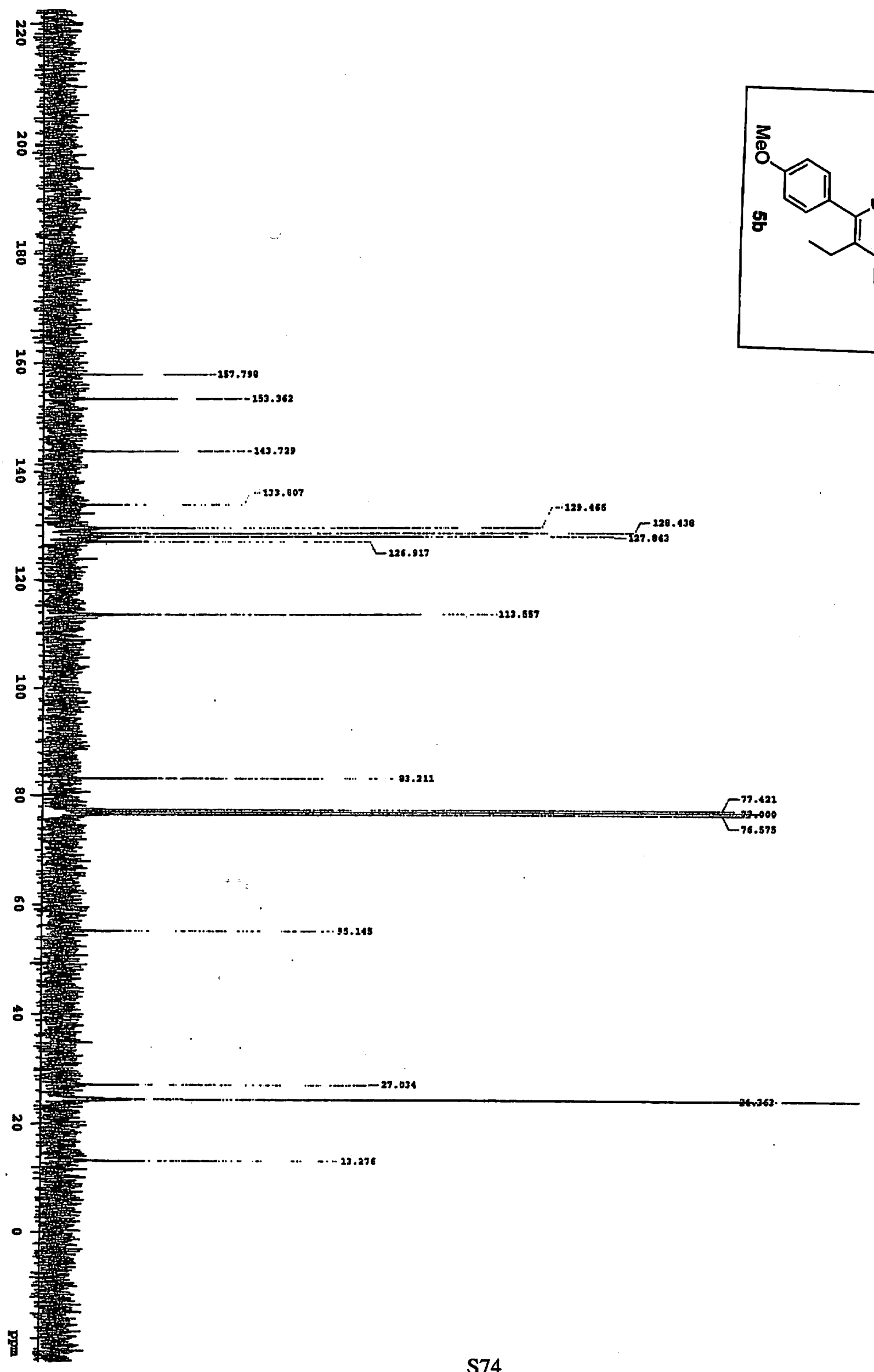




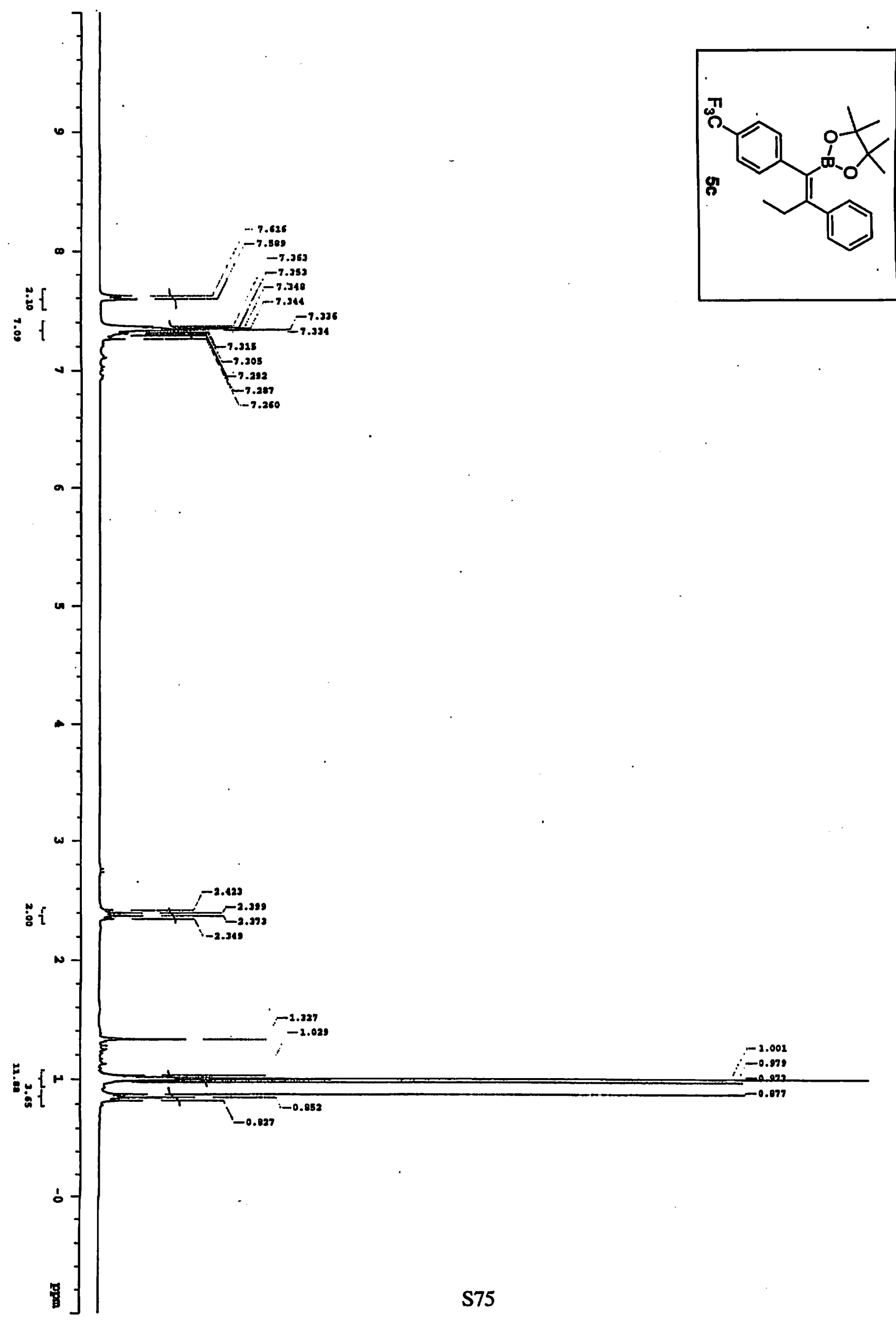




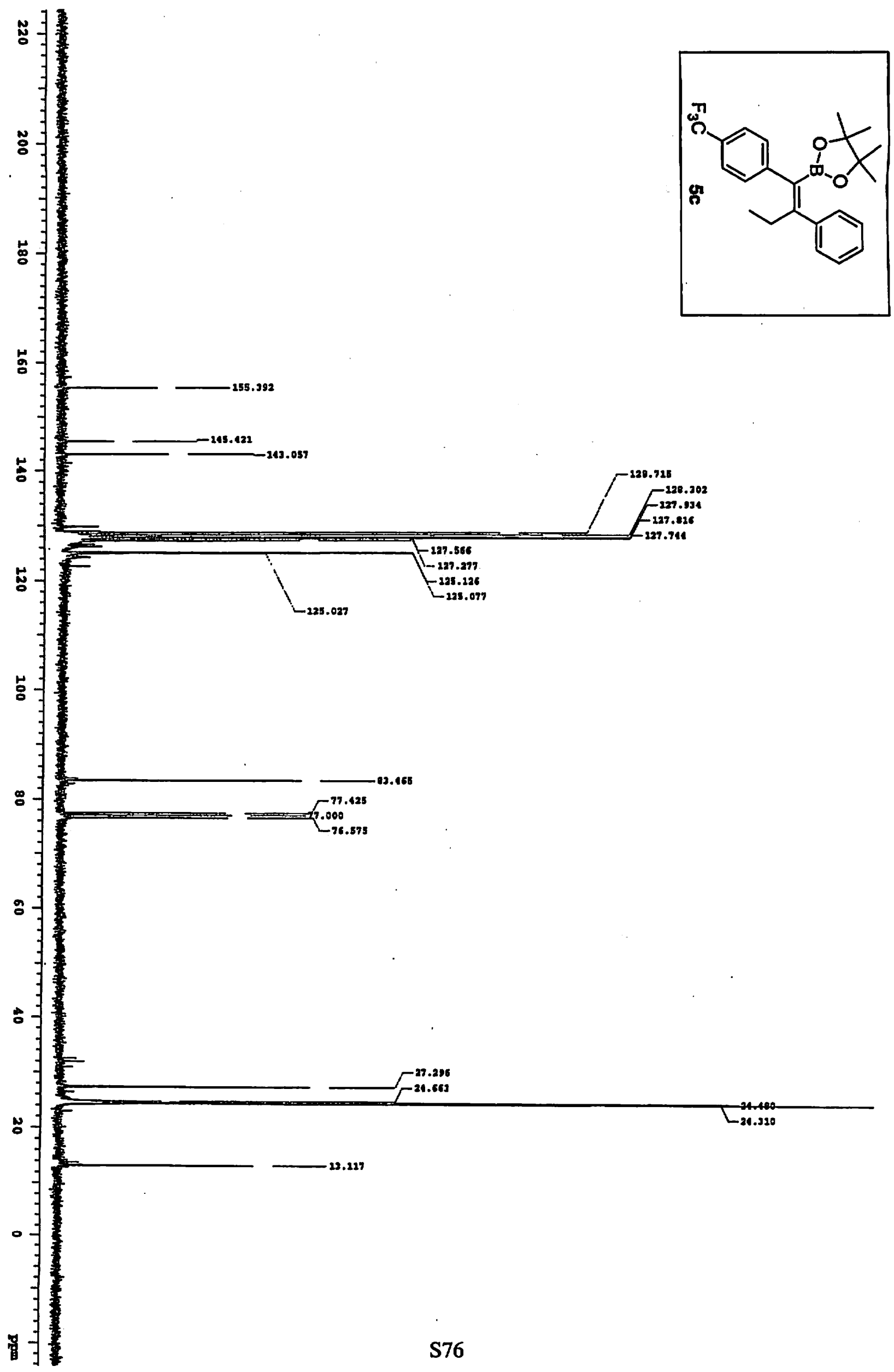




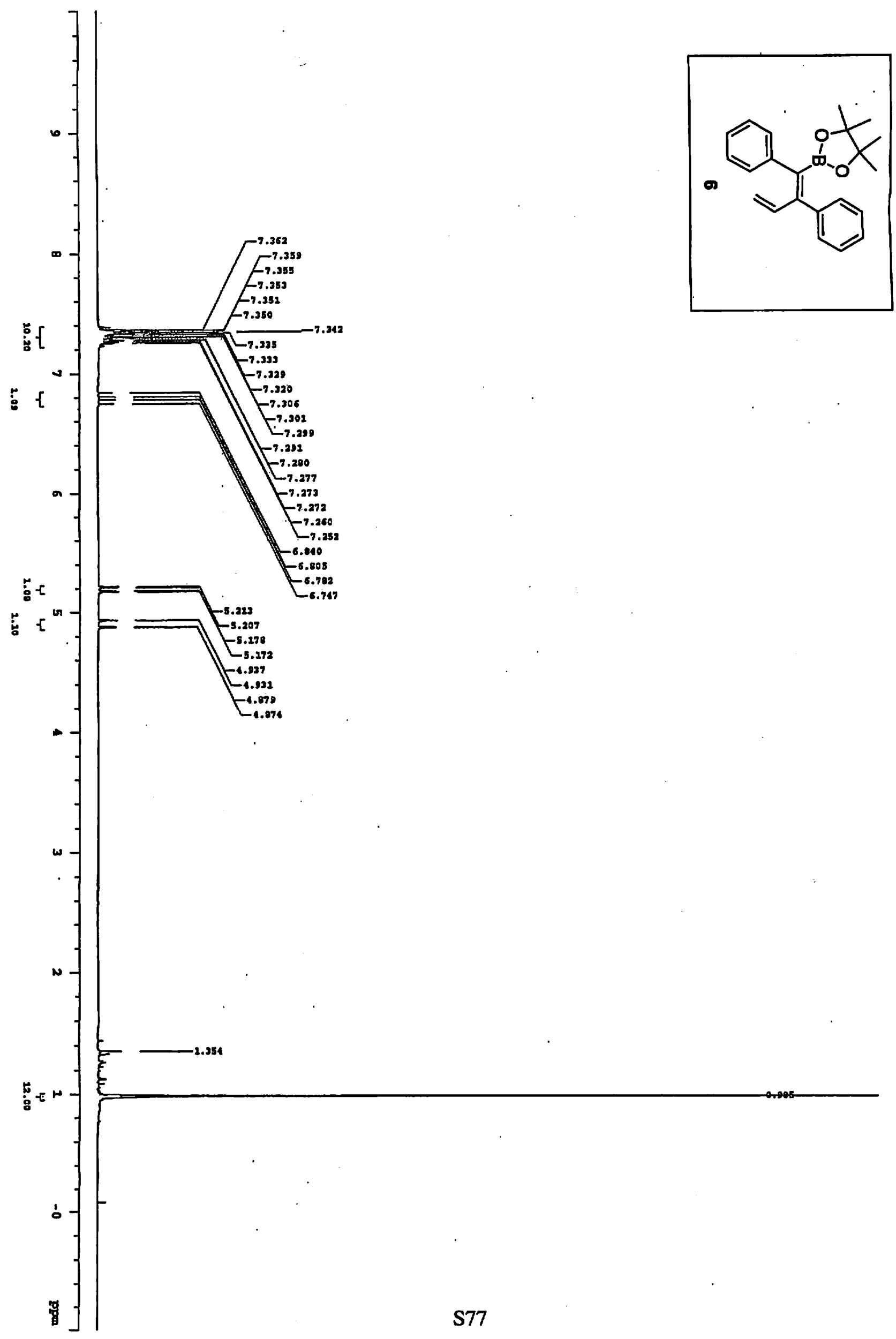




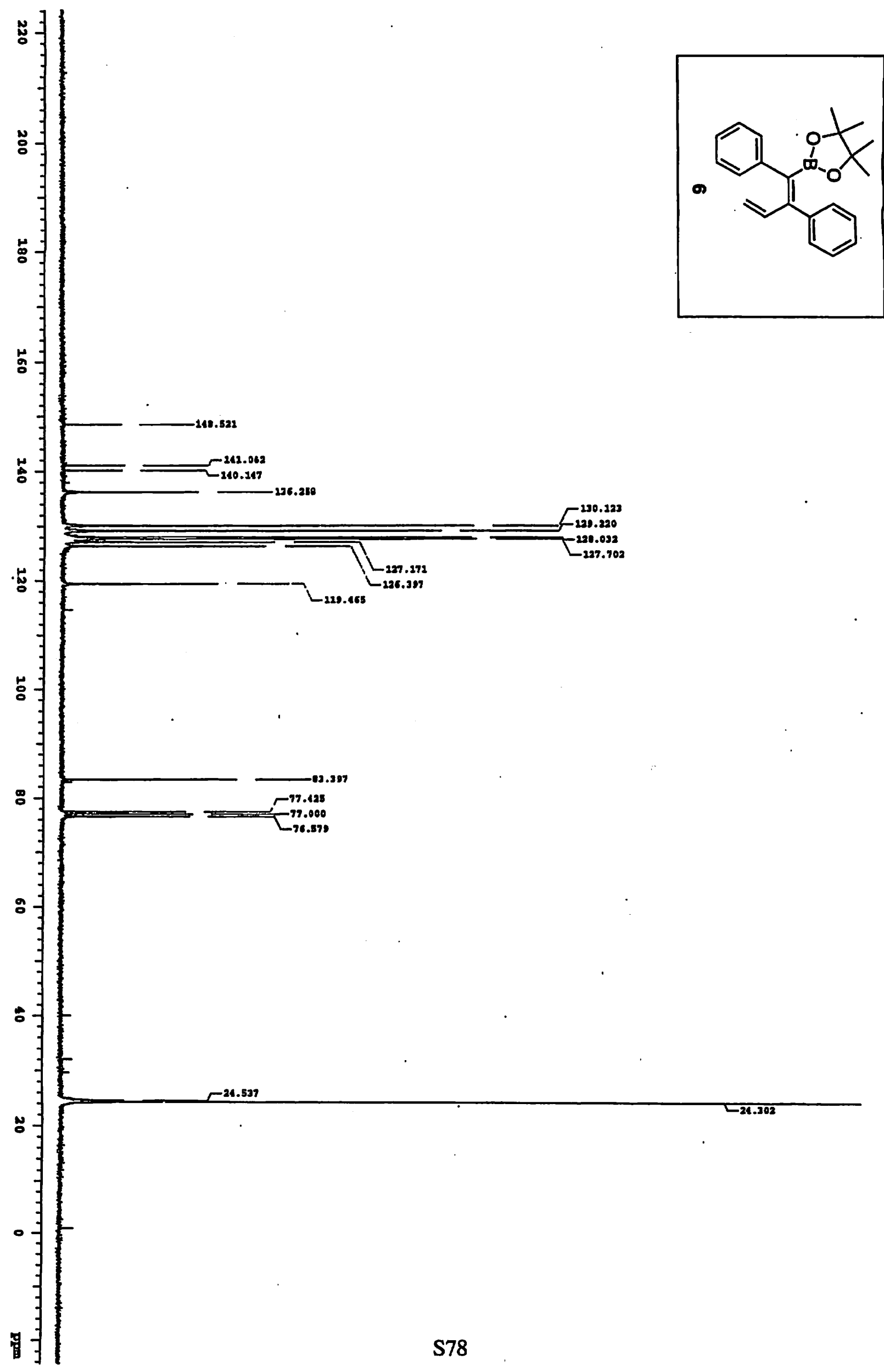




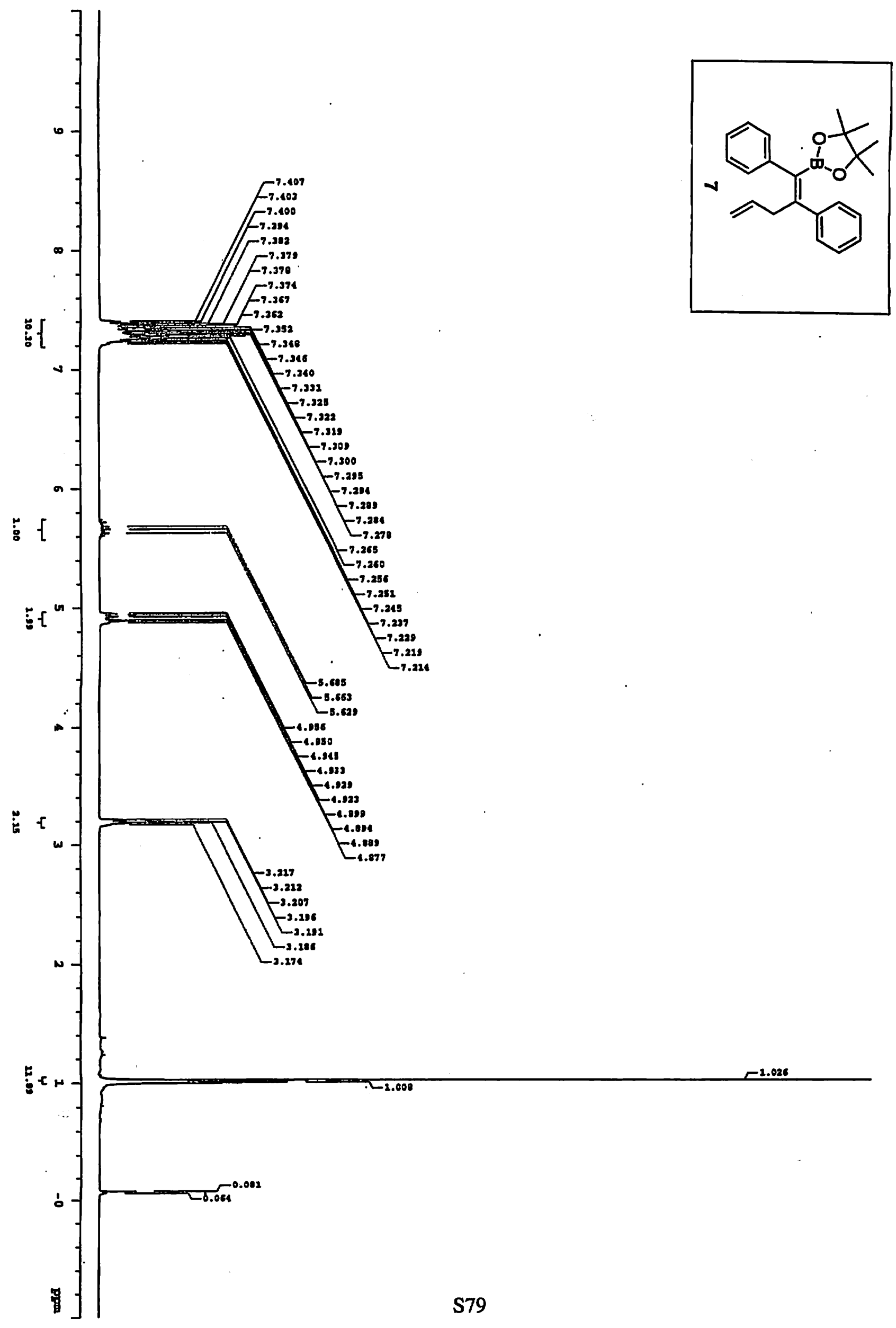




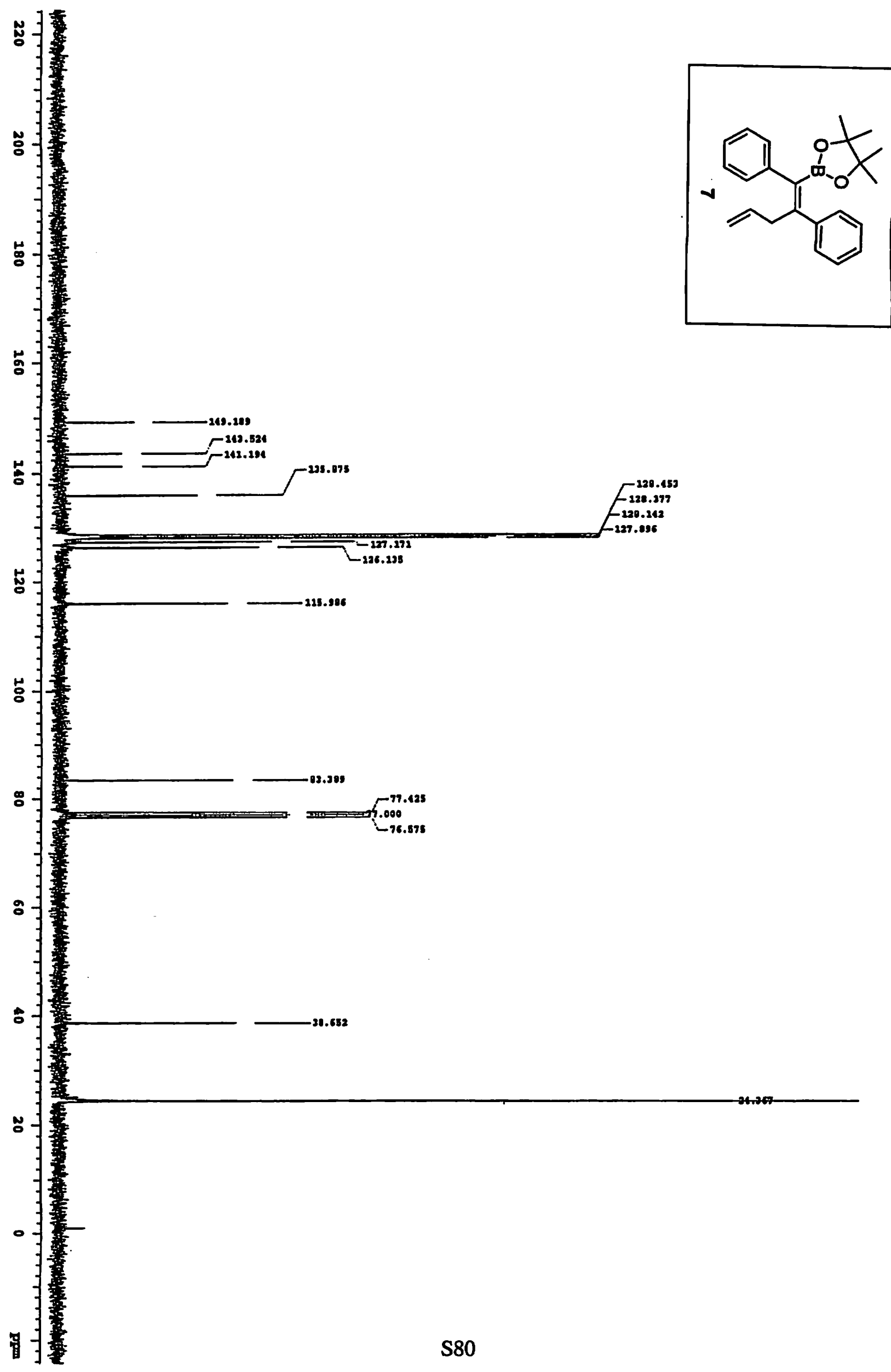




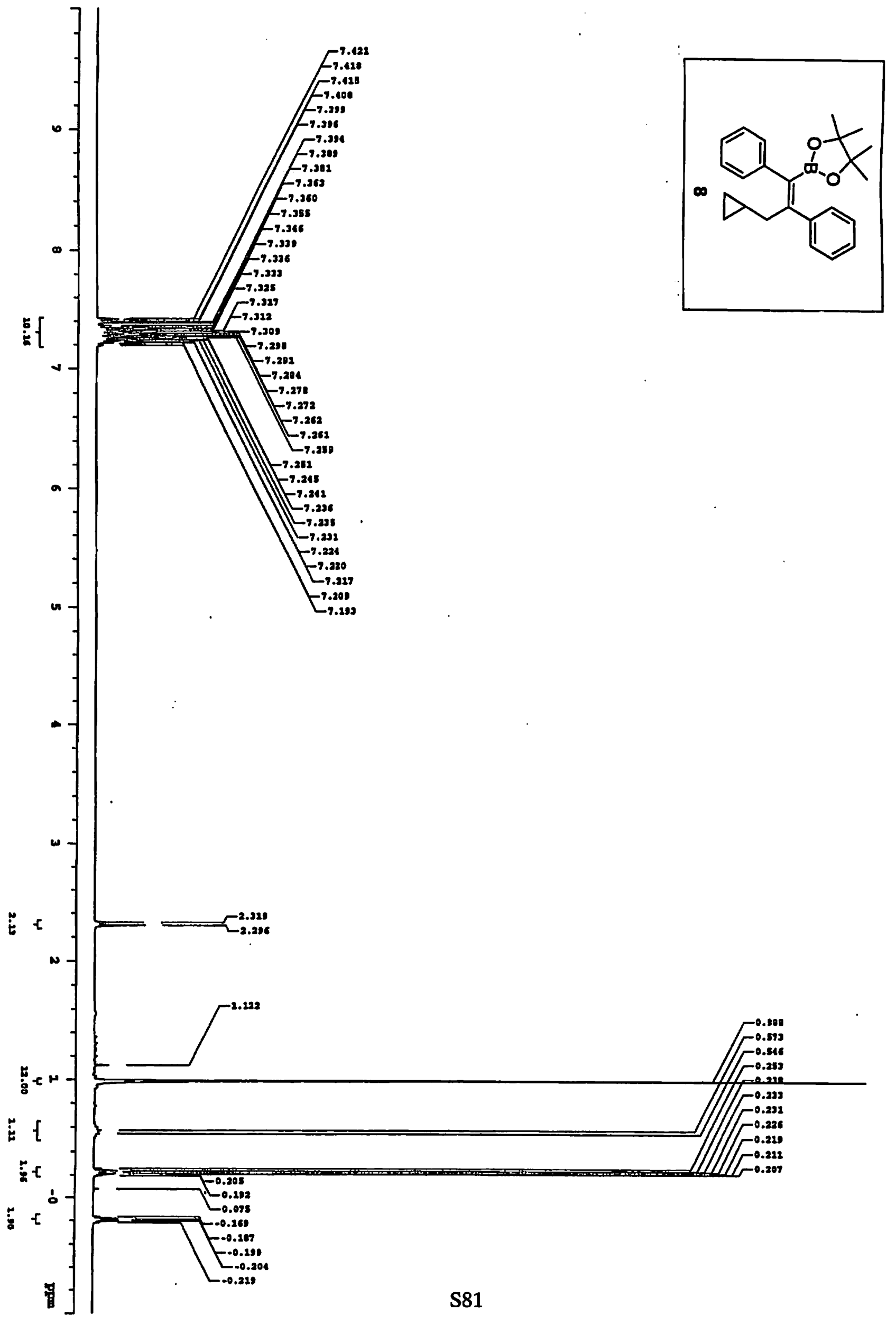




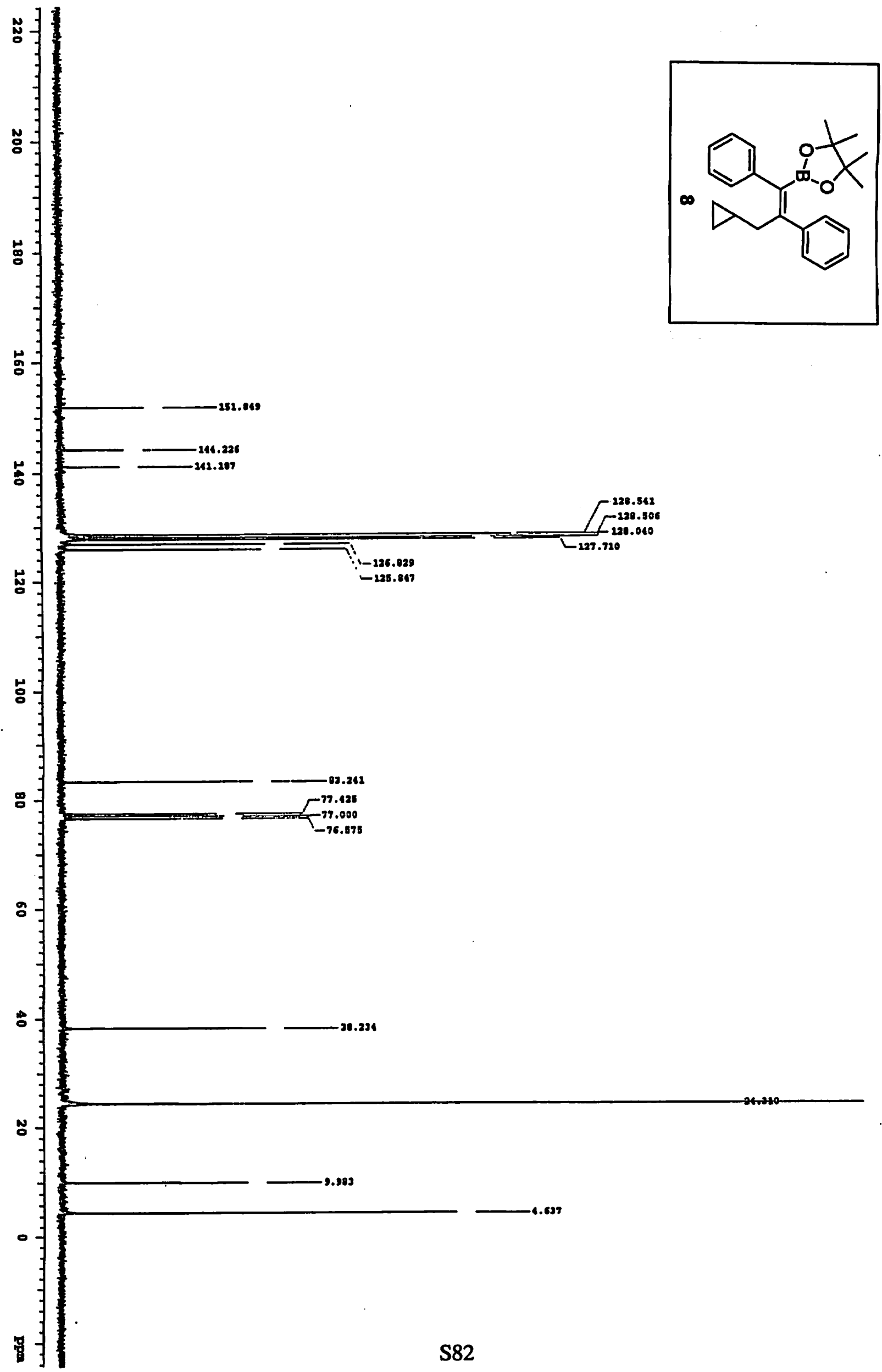




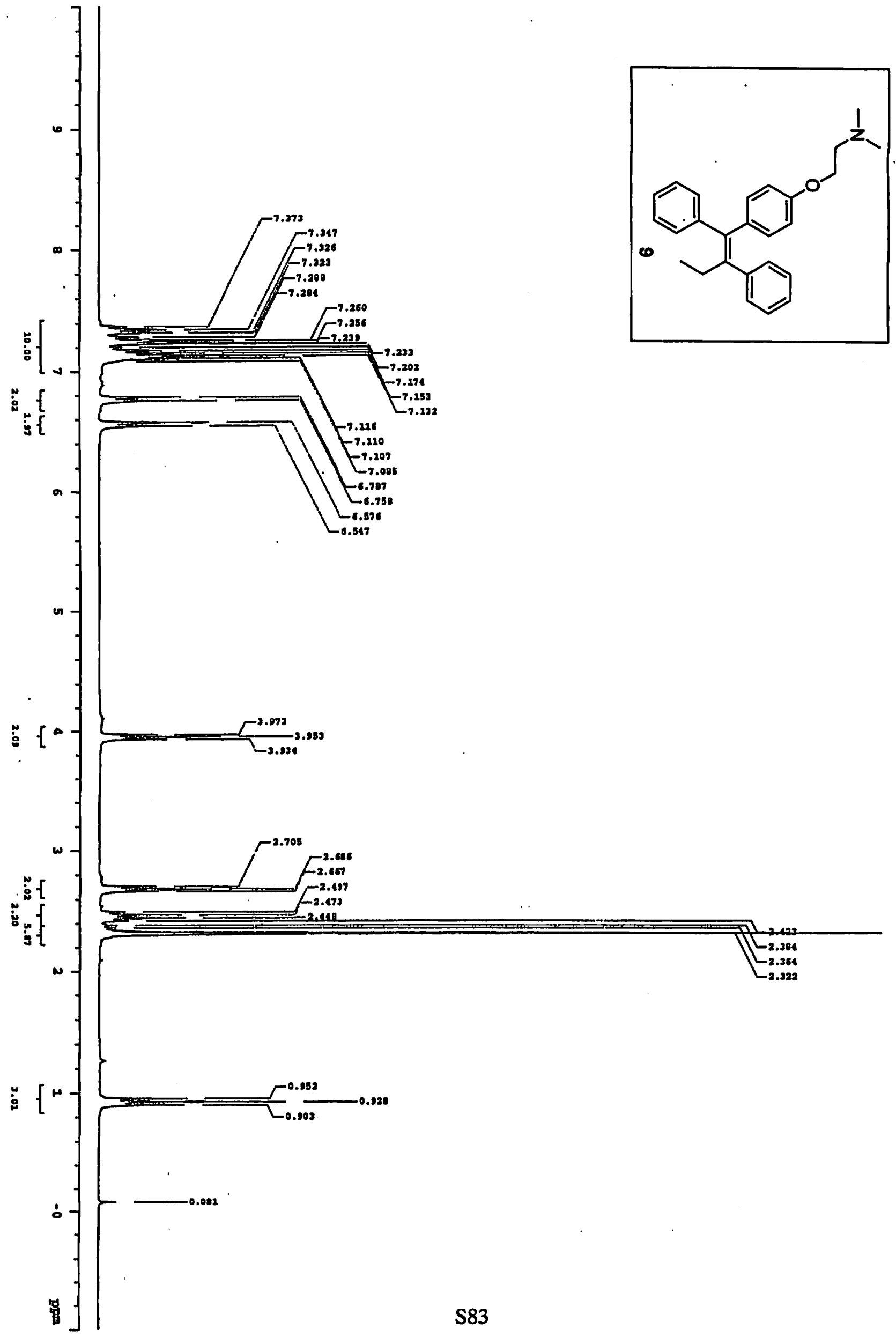




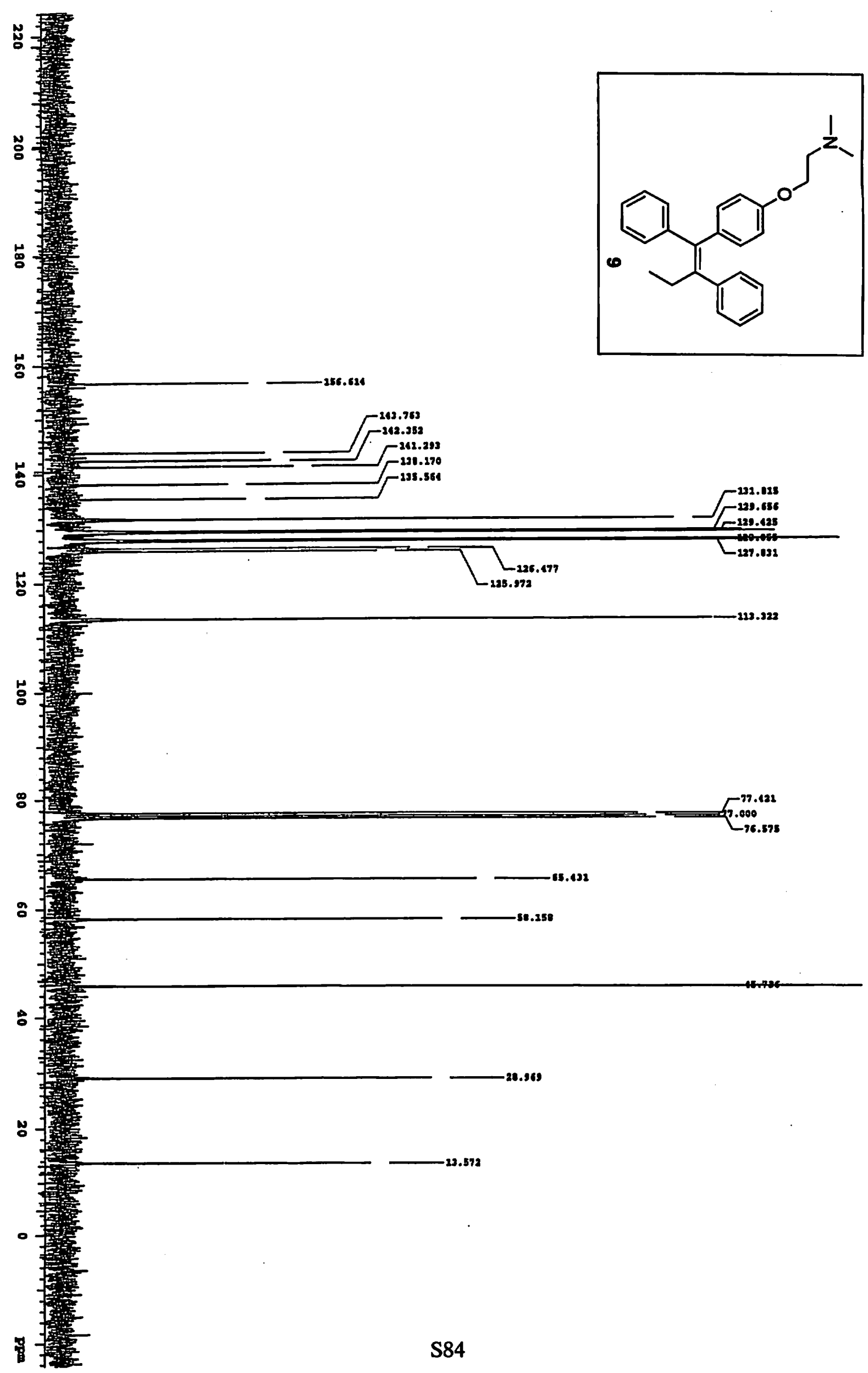

